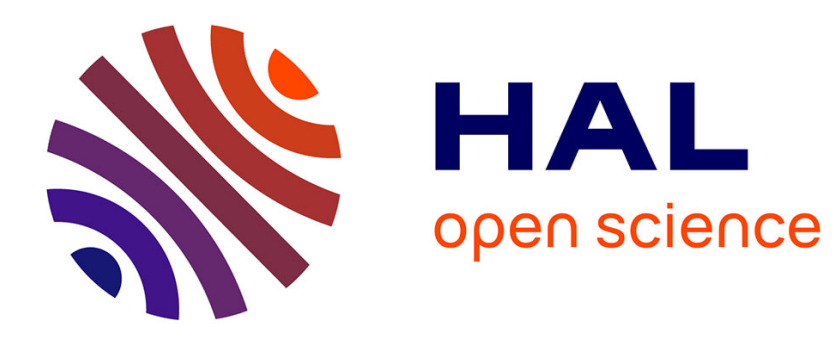

\title{
Kantorovich-Rubinstein Quasi-Metrics II: Hyperspaces and Powerdomains
}

\author{
Jean Goubault-Larrecq
}

\section{To cite this version:}

Jean Goubault-Larrecq. Kantorovich-Rubinstein Quasi-Metrics II: Hyperspaces and Powerdomains. Topology and its Applications, 2022, 305, pp.107885. 10.1016/j.topol.2021.107885 . hal-03383036

\section{HAL Id: hal-03383036 \\ https://hal.inria.fr/hal-03383036}

Submitted on 18 Oct 2021

HAL is a multi-disciplinary open access archive for the deposit and dissemination of scientific research documents, whether they are published or not. The documents may come from teaching and research institutions in France or abroad, or from public or private research centers.
L'archive ouverte pluridisciplinaire HAL, est destinée au dépôt et à la diffusion de documents scientifiques de niveau recherche, publiés ou non, émanant des établissements d'enseignement et de recherche français ou étrangers, des laboratoires publics ou privés. 


\title{
Kantorovich-Rubinstein Quasi-Metrics II: Hyperspaces and Powerdomains
}

\author{
Jean Goubault-Larrecq \\ Université Paris-Saclay, ENS Paris-Saclay, CNRS, LSV, 91190, Gif-sur-Yvette, France. \\ goubault@lsv.fr
}

\begin{abstract}
We show that the Kantorovich-Rubinstein quasi-metrics $d_{\mathrm{KR}}$ and $d_{\mathrm{KR}}^{a}$ of Part I extend naturally to various spaces of previsions, and in particular not just the linear previsions (roughly, measures) of Part I. There are natural isomorphisms between the Hoare and Smyth powerdomains, as used in denotational semantics, and spaces of discrete sublinear previsions, and of discrete normalized superlinear previsions, respectively. Turning to the corresponding hyperspaces, namely the same powerdomains, but equipped with the lower Vietoris and upper Vietoris topologies instead, this turns into homeomorphisms with the corresponding space of previsions, equipped with the so-called weak topology. Through these isomorphisms again, the two powerdomains inherit quasi-metrics $d_{\mathcal{H}}$ and $d_{\mathcal{Q}}$, respectively, that are reminiscent of the well-known Hausdorff metric. Then we show that the Hoare and Smyth powerdomains of an algebraic complete quasi-metric space are again algebraic complete, with those quasi-metrics, and similarly that the corresponding powerdomains of continuous complete quasi-metric spaces are continuous complete. Furthermore, in the continuous complete case, the $d_{\mathcal{H}^{-}}$ Scott topology coincides with the lower Vietoris topology, and the $d_{\mathcal{Q}}$-Scott topology coincides with the upper Vietoris topology.
\end{abstract}

Keywords: Quasi-metric, prevision, powerdomain (Hoare, Smyth), hyperspace, Kantorovich-Rubinstein quasi-metric, Vietoris topology (lower, upper) 2020 MSC: 54E99, 54H30 


\section{Introduction}

This is a sequel to [12], where we explored quasi-metric variants of the Kantorovich-Rubinstein metric on spaces of measures, or rather of continuous valuations. The basic variant, $d_{\mathrm{KR}}$, is defined by the formula:

$$
d_{\mathrm{KR}}\left(\nu, \nu^{\prime}\right) \stackrel{\text { def }}{=} \sup _{h \in \mathcal{L}_{1} X} \max \left(\int_{x \in X} h(x) d \nu-\int_{x \in X} h(x) d \nu^{\prime}, 0\right) .
$$

where $\mathcal{L}_{1} X$ is the collection of 1-Lipschitz continuous maps from a given quasi-metric space $X, d$ to $\overline{\mathbb{R}}_{+}=\mathbb{R}_{+} \cup\{+\infty\}$, and $\nu$ and $\nu^{\prime}$ are two continuous valuations on $X$. (We also agree that $(+\infty)-(+\infty)=0$, and that $(+\infty)-t=$ $+\infty$ for every $t<+\infty$.) This is close to the usual Kantorovich-Rubinstein metric, in which we would write an absolute value sign instead of $\max \left({ }_{-}, 0\right)$; in fact, if the chosen quasi-metric $d$ on $X$ is a metric, then $d_{\mathrm{KR}}$ is also a metric, and changing $\max (,, 0)$ into an absolute value in (1) would not make a difference [12, Equation (12)].

For every continuous valuation $\nu$ on $X$, the map $G: h \mapsto \int_{x \in X} h(x) d \nu$ is a Scott-continuous and linear functional, and one may rewrite (1) as follows:

$$
d_{\mathrm{KR}}\left(G, G^{\prime}\right)=\sup _{h \in \mathcal{L}_{1} X} \max \left(G(h)-G^{\prime}(h), 0\right) .
$$

This makes sense for every pair of functionals $G, G^{\prime}$. The goal of this paper and of its sequels is to explore properties of this quasi-metric on certain spaces of functionals on $X$, called previsions in [6], which are not necessarily linear, and which are used to give semantics to mixed, probabilistic and non-deterministic forms of choice in programming languages ${ }^{1}$.

Outline. We recapitulate some preliminary definitions and notions in Section 2. This is copied almost verbatim from Section 2 of Part I [12].

In this paper, we will deal with the purely non-deterministic forms of choice, although we will also prepare the grounds for the mixed non-deterministic and probabilistic forms of Part III and Part IV, by stating a few completeness theorems about $d_{\mathrm{KR}}$ on general spaces of previsions in Section 4 . Those are natural extensions of similar theorems given in Part I for linear previsions, or equivalently, continuous valuations [12].

\footnotetext{
${ }^{1}$ An unpublished version of that work is available on arXiv [9]. The present paper roughly covers Sections $10.1,10.2,10.3,12$, and 13.
} 
There are three canonical powerdomains: the Hoare, Smyth and Plotkin powerdomains. We will concentrate on the first two. The Plotkin powerdomain will be dealt with in Part IV.

The Hoare powerdomain $\mathcal{H} X$ serves to model angelic non-determinism, and consists of all the (non-empty) closed subsets of $X$, ordered by inclusion. The Smyth powerdomain $\mathcal{Q} X$ serves to model demonic non-determinism, and consists of all the non-empty compact saturated subsets of $X$. We will see in Section 3 that there is a natural way to associate a prevision $F^{C}$ with every closed subset $C$, and a prevision $F_{Q}$ with every element $Q$ of $\mathcal{Q} X$, so that $\mathcal{H} X$ and $\mathcal{Q} X$ are in bijection with certain spaces of so-called discrete previsions.

The Hoare hyperspace is $\mathcal{H} X$ with the so-called lower Vietoris topology, and we will see that the bijection we just alluded to turns into a homeomorphism between the Hoare hyperspace and a certain space of previsions with the so-called weak topology. A similar phenomenon will happen with the Smyth hyperspace.

The above bijection allows us to transport the $d_{\mathrm{KR}}$ quasi-metric, and a bounded variant $d_{\mathrm{KR}}^{a}$, to certain quasi-metrics on the Hoare and Smyth hyperspaces, and those quasi-metrics will be our main object of study.

In order to study them, we first study $d_{\mathrm{KR}}$ and $d_{\mathrm{KR}}^{a}$ in a general setting in Section 4. Notably, we show that the space $\mathbf{P} X$ of previsions on a quasimetric space $X, d$ is complete with respect to $d_{\mathrm{KR}}$, resp. $d_{\mathrm{KR}}^{a}$, either when $X, d$ is Lipschitz regular (Theorem 4.8 and Theorem 4.16 - the Lipschitz regular case is probably not that interesting per se, but is a crucial technical tool in our developments), or under a certain condition on supports (Proposition 4.25). The latter condition will be discharged on a case-by-case basis in subsequent sections, in Part III and in Part IV.

We deal with the Hoare hyperspace, specifically, in Section 5. We introduce a quasi-metric $d_{\mathcal{H}}$, defined by $d_{\mathcal{H}}\left(C, C^{\prime}\right) \stackrel{\text { def }}{=} \sup _{x \in C} d\left(x, C^{\prime}\right)$. In metric spaces, $d\left(x, C^{\prime}\right)=\inf _{y \in C^{\prime}} d(x, y)$, and therefore $d_{\mathcal{H}}\left(C, C^{\prime}\right)=\sup _{x \in C} \inf _{y \in C^{\prime}} d(x, y)$, which shows that $d_{\mathcal{H}}$ is essentially one half of the definition of the Hausdorff metric; in quasi-metric spaces, $d\left(x, C^{\prime}\right)$ has a slightly different definition. We will see that the bijection $C \mapsto F^{C}$ between $\mathcal{H} X$ and a certain set of discrete previsions induces an isometry that relates $d_{\mathcal{H}}$ with $d_{\mathrm{KR}}$, and that $\mathcal{H} X, d_{\mathcal{H}}$ is algebraic complete if $X, d$ is algebraic complete, and continuous complete if $X, d$ is continuous complete. Finally, we will show that, in those cases, the $d_{\mathcal{H}}$-Scott topology on $\mathcal{H} X$ coincides with the so-called lower Vietoris 
topology.

The Smyth hyperspace is the subject of Section 6, and will be dealt in an entirely similar fashion. There $d_{\mathrm{KR}}$ will match a quasi-metric $d_{\mathcal{Q}}$, which can be thought of being the other half of the Hausdorff metric.

\section{Preliminaries}

\subsection{General topology}

We refer the reader to [7] for basic notions and theorems of topology, domain theory, and in the theory of quasi-metric spaces. The book [5] is the standard reference on domain theory, and I will assume known the notions of directed complete posets (dcpo), Scott-continuous functions, the way-below relation $\ll$, continuous posets and dcpos, and so on. Additionally, this paper will heavily rely on [13] for additional results on quasi-metric spaces, on [10] for formal ball monads, and on [11] for results on spaces of Lipschitz continuous maps; we will recall the required results as needed.

A family $D$ in a poset is directed if and only if it is non-empty, and every pair of elements of $D$ has an upper bound in $D$. Symmetrically, a filtered family is a non-empty family $D$ such that every pair of elements of $D$ has a lower bound in $D$.

The Scott topology on a poset consists of the Scott-open subsets, namely the upwards-closed subsets $U$ such that every directed family that has a supremum in $U$ must intersect $U$. A Scott-continuous map between posets is one that is monotonic and preserves existing directed suprema, and this is equivalent to requiring that it is continuous for the underlying Scott topologies.

The focus of the present paper is on quasi-metric spaces. Chapters 6 and 7 of [7] are a recommended read on that subject. The paper [13] gives additional information on quasi-metric spaces, which we will also rely on.

As far as topology is concerned, compactness does not imply separation. In other words, we call a subset $K$ of a topological space compact if and only if every open cover of $K$ contains a finite subcover. This property is sometimes called quasicompactness.

We shall always write $\leq$ for the specialization preordering of a topological space: $x \leq y$ if and only if every open neighborhood of $x$ is also an open neighborhood of $y$, if and only if $x$ is in the closure of $y$. As a result, the closure of a single point $y$ is also its downward closure $\downarrow y$. In general, we write $\downarrow A$ for the downward closure of any set $A, \uparrow A$ for its upward closure, 
and $\uparrow x$ for $\uparrow\{x\}$. A saturated subset of a topological space is a set that is the intersection of all its open neighborhoods; equivalently, it is an upwardsclosed subset in its specialization preordering.

In a topological space, $\uparrow A$ is also equal to the saturation of $A$, namely the intersection of all the open neighborhoods of $A$. If $K$ is compact, then so is its saturation $\uparrow K$, and we will usually use the letter $Q$ for compact saturated subsets.

\subsection{Quasi-metric spaces}

Let $\overline{\mathbb{R}}_{+}$be the set of extended non-negative reals. A quasi-metric on a set $X$ is a map $d: X \times X \rightarrow \overline{\mathbb{R}}_{+}$satisfying: $d(x, x)=0 ; d(x, z) \leq d(x, y)+d(y, z)$ (triangular inequality); $d(x, y)=d(y, x)=0$ implies $x=y$. The pair $X, d$ is then called a quasi-metric space. Given $X, d$, there is an ordering $\leq^{d}$ on $X$ given by $x \leq^{d} y$ if and only if $d(x, y)=0$.

A trivial example is $\overline{\mathbb{R}}_{+}$itself, with the quasi-metric $\mathrm{d}_{\mathbb{R}}$ defined by $\mathrm{d}_{\mathbb{R}}(x, y) \stackrel{\text { def }}{=}$

0 if $x \leq y, \mathrm{~d}_{\mathbb{R}}(+\infty, y) \stackrel{\text { def }}{=}+\infty$ if $y \neq+\infty, \mathrm{d}_{\mathbb{R}}(x, y) \stackrel{\text { def }}{=} x-y$ if $x>y$ and $x \neq+\infty$. Then $\leq^{\mathrm{d}_{\mathbb{R}}}$ is the usual ordering $\leq$.

The space of formal balls $\mathbf{B}(X, d)$ of a quasi-metric space $X, d$ is probably the single most important artifact that has to be considered in the study of quasi-metric spaces $[17,3]$. This has a very simple definition: a formal ball is syntax for an actual ball, namely a pair $(x, r)$ where $x \in X$ (the center) and $r \in \mathbb{R}_{+}$(the radius). $\mathbf{B}(X, d)$ comes with an ordering $\leq{ }^{d^{+}}$defined by $(x, r) \leq d^{+}(y, s)$ if and only if $d(x, y) \leq r-s$.

Given a quasi-metric space $X, d$, the open ball $B_{x,<r}^{d}$ with center $x \in X$ and radius $r \in \mathbb{R}_{+}$is $\{y \in X \mid d(x, y)<r\}$. The open ball topology is the coarsest that contains all open balls, and is the standard topology on metric spaces.

In the realm of quasi-metric spaces, the $d$-Scott topology is the topology we shall always consider, unless specified otherwise. This is defined as follows. We equip $\mathbf{B}(X, d)$ with the Scott topology of $\leq^{d^{+}}$. There is an injective map $\eta_{X}: x \mapsto(x, 0)$ from $X$ to $\mathbf{B}(X, d)$, and the $d$-Scott topology is the coarsest topology that makes it continuous. This allows us to see $X$ as a topological subspace of $\mathbf{B}(X, d)$.

The specialization ordering of $X, d$, whether with the open ball topology or with the $d$-Scott topology, is just $\leq d$. This does not cause any conflict with the notation $\leq d^{d^{+}}$for the ordering on $\mathbf{B}(X, d)$, since $\leq^{d^{+}}$is in fact the ordering 
deduced from a quasi-metric $d^{+}$on $\mathbf{B}(X, d)$, defined by $d^{+}((x, r),(y, s)) \stackrel{\text { def }}{=}$ $\max (d(x, y)-r+s, 0)$.

The $d$-Scott topology coincides with the familiar open ball topology when $d$ is a metric [7, Proposition 7.4.46], or when $X, d$ is Smyth-complete [7, Proposition 7.4.47]. It coincides with the generalized Scott topology of [1] when $X, d$ is an algebraic complete quasi-metric space [7, Exercise 7.4.69] (see below for the definition of "algebraic complete"). On $\overline{\mathbb{R}}_{+}$, the $\mathbb{d}_{\mathbb{R}}$-Scott topology coincides with the Scott topology of the usual ordering $\leq$; its non-trivial opens are the open intervals $] r,+\infty], r \in \mathbb{R}_{+}$. The $d^{+}$-Scott topology also coincides with the familiar Scott topology on $\mathbf{B}(X, d)$, see [10, Lemma 3.6] or [7, Exercise 7.4.53].

We say that $X, d$ is complete (or Yoneda-complete) if and only if $\mathbf{B}(X, d)$ is a dcpo. We take this as a definition, although this is really a theorem, due to Kostanek and Waszkiewicz, see [15] and [7, Theorem 7.4.27].

Outside complete spaces, we would like to avoid certain pathologies and we will therefore concentrate on standard quasi-metric spaces [13, Section 2]. $X, d$ is standard if and only if, for every directed family of formal balls $\left(x_{i}, r_{i}\right)_{i \in I}$, for every $s \in \mathbb{R}_{+},\left(x_{i}, r_{i}\right)_{i \in I}$ has a supremum in $\mathbf{B}(X, d)$ if and only if $\left(x_{i}, r_{i}+s\right)_{i \in I}$ has a supremum in $\mathbf{B}(X, d)$. Writing the supremum of the former as $(x, r)$, we then have that $r=\inf _{i \in I} r_{i}$, and the supremum of the latter is $(x, r+s)$ - this holds not only for $s \in \mathbb{R}_{+}$, but for every $s \geq-r$. If $X, d$ is standard, then the radius map $(x, r) \mapsto r$ is Scott-continuous from $\mathbf{B}(X, d)$ to $\overline{\mathbb{R}}_{+}^{o p}\left(\overline{\mathbb{R}}_{+}\right.$with the opposite ordering $\left.\geq\right)$, and for every $s \in \mathbb{R}_{+}$, the map _ $+s:(x, r) \mapsto(x, r+s)$ is Scott-continuous from $\mathbf{B}(X, d)$ to itself [13, Proposition 2.4]. Moreover, and we will use that fact several times, the set $V_{\epsilon} \stackrel{\text { def }}{=}\{(x, r) \in \mathbf{B}(X, d) \mid r<\epsilon\}$ is Scott-open for every $\epsilon>0$, whenever $X, d$ is standard. This is because $V_{\epsilon}$ is the inverse image of the Scott-open subset $\left[0, \epsilon\left[\right.\right.$ of $\overline{\mathbb{R}}_{+}^{o p}$ by the radius map.

All complete quasi-metric spaces are standard. Also, all metric spaces are standard. Even posets $X$, which can all be seen as quasi-metric spaces $X, d_{\leq}$, by defining $d_{\leq}(x, y)$ as 0 if $x \leq y,+\infty$ otherwise, are all standard. Note that $\overline{\mathbb{R}}_{+}, \mathrm{d}_{\mathbb{R}}$ is standard, being complete.

Directed complete posets (dcpos) come into further varieties: algebraic dcpos, continuous dcpos, notably, and that extends to algebraic posets and continuous posets. The same happens with quasi-metric spaces. We say that $X, d$ is a continuous quasi-metric space if any only it is standard and $\mathbf{B}(X, d)$ is a continuous poset. Hence it is continuous complete if and only if $\mathbf{B}(X, d)$ 
is a continuous dcpo. The latter is originally a theorem, not a definition [15].

For the sake of completeness, let us recall what a continuous poset is. The way-below relation $\ll$ on a poset is defined by $x \ll y$ if and only if every directed family that has a supremum above $y$ contains an element above $x$. (We will often say "above" for "larger than or equal to", and similarly for "below".) A basis of a poset is a subset $\mathcal{B}$ such that every point $x$ of the poset is the supremum of a directed family of elements of $\mathcal{B}$. A continuous poset is a poset with a basis. In that case, the poset itself is a basis, namely the largest one. We write $\uparrow x$ for the set of points $y$ such that $x \ll y$. If $\mathcal{B}$ is a basis of a poset $X$, the sets $\uparrow x$ with $x \in \mathcal{B}$ form a base of the Scott topology.

A point $x \in X$ is a center point of a quasi-metric space $X, d$ if and only if $B_{(x, 0), \epsilon}^{d^{+}}$is Scott-open in $\mathbf{B}(X, d)$ for every $\epsilon>0$. The notion coincides with that of a $d$-finite point, provided that $X, d$ is standard, see Lemma 5.7 of [13]. A standard quasi-metric space $X, d$ is algebraic if and only if every point is a $d$-limit of a Cauchy net of $d$-finite points, or equivalently of a Cauchyweightable net of $d$-finite points - see the comments after Example 5.3 of [13] — or equivalently, if and only if for every $x \in X$, there is a directed family $\left(x_{i}, r_{i}\right)_{i \in I}$ of formal balls such that each $x_{i}$ is a center point and whose supremum is $(x, 0)$. Yet another equivalent definition is: a standard quasimetric space $X, d$ is algebraic if and only if the open balls $B_{(x, 0), \epsilon}^{d^{+}}$, where $x$ ranges over the center points and $\epsilon>0$, form a base of the Scott topology on $\mathbf{B}(X, d)$ [13, Definition 5.11, Theorem 5.16]. Every (standard) algebraic quasi-metric space is continuous. Moreover, for every center point $z$, we have $(z, t) \ll(y, s)$ if and only if $d(z, y)<t-s$; more generally, $(y, s) \ll(x, r)$ if and only if there is a center point $z$ and some $t \in \mathbb{R}_{+}$such that $(y, s) \leq{ }^{d^{+}}(z, t)$ and $d(z, x)<t-r$ [13, Proposition 5.18].

In a metric space, those complications vanish, as every point is a center point. Every point is a center point in a Smyth-complete space as well, but we will not be concerned with Smyth-completeness. One has to be careful with $\overline{\mathbb{R}}_{+}, \mathrm{d}_{\mathbb{R}}$ already: the center points of $\overline{\mathbb{R}}_{+}, \mathrm{d}_{\mathbb{R}}$ are the elements of $\mathbb{R}_{+}$, but not $+\infty$. Indeed, $B_{(+\infty, 0),<\epsilon}^{\mathrm{d}_{\mathbb{R}}}$ is the set of formal balls $(+\infty, r)$ with $r<\epsilon$, and that is not Scott-open, since $(+\infty, 0)$ for example is the supremum of the chain $\left\{(t, 0) \mid t \in \mathbb{R}_{+}\right\}$, but no point $(t, 0)$ with $t \in \mathbb{R}_{+}$is in $B_{(+\infty, 0),<\epsilon}^{\mathrm{d}_{\mathbb{R}}}$. The space $\overline{\mathbb{R}}_{+}, \mathrm{d}_{\mathbb{R}}$ is algebraic complete, with $\mathbb{R}_{+}$as set of center points.

Given a $d$-Scott open subset $U$ of $X$, there is a largest Scott open subset $\widehat{U}$ of $\mathbf{B}(X, d)$ such that $U=\widehat{U} \cap X$. (We silently equate $X$ with a subspace of $\mathbf{B}(X, d)$.) The assignment $U \mapsto \widehat{U}$ is monotonic; being left-adjoint 
to the map $V \mapsto V \cap X$, it preserves arbitrary meets (namely, interiors of intersections), but it satisfies no other remarkable property in general. A quasi-metric space $X, d$ is Lipschitz regular if and only if that assignment is Scott-continuous. This is defined and studied in Section 4 of [10]. If that is the case, then $X, d$ is finitarily embedded in $\mathbf{B}(X, d)$; see [4] for more information on finitary embeddings. For algebraic complete quasi-metric spaces, Lipschitz regularity is equivalent to having relatively compact balls, a stronger requirement than local compactness. This displays Lipschitz regularity as a rather strong requirement. In spite of this, the space of formal balls $\mathbf{B}(X, d)$ of any quasi-metric space $X, d$, with the $d^{+}$quasi-metric, is Lipschitz regular [10, Theorem 4.12].

Given a map $f$ from a quasi-metric space $X, d$ to a quasi-metric space $Y, \partial, f$ is $\alpha$-Lipschitz if and only if $\partial(f(x), f(y)) \leq \alpha d(x, y)$ for all $x, y \in X$. (When $\alpha=0$ and $d(x, y)=+\infty$, we take the convention that 0 times $+\infty$ equals 0 .)

For every $\alpha \in \mathbb{R}_{+}$, and every map $f: X, d \rightarrow Y, \partial$, let $\mathbf{B}^{\alpha}(f) \operatorname{map}(x, r) \in$ $\mathbf{B}(X, d)$ to $(f(x), \alpha r) \in \mathbf{B}(Y, \partial)$. Then $f$ is $\alpha$-Lipschitz if and only if $\mathbf{B}^{\alpha}(f)$ is monotonic.

Contrarily to the case of spaces with the open ball topology, a Lipschitz map need not be continuous. For example, on posets seen as quasi-metric spaces through $d(x, y)=0$ if $x \leq y,+\infty$ otherwise, the Lipschitz maps are the monotonic maps [13, Example 6.1], and the continuous maps, with respect to the underlying $d$-Scott topologies, are the Scott-continuous maps.

We say that a map $f: X, d \rightarrow Y, \partial$ between quasi-metric spaces is $\alpha$ Lipschitz continuous if and only if $\mathbf{B}^{\alpha}(f)$ is Scott-continuous [10, Definition 2.3]. While " $\alpha$-Lipschitz continuous" should be thought of as one epithet, not as the conjunction of " $\alpha$-Lipschitz" and "continuous" in general, we have the following. When both $X, d$ and $Y, \partial$ are standard, $f: X \rightarrow Y$ is $\alpha$-Lipschitz continuous if and only if $f$ is both $\alpha$-Lipschitz, and continuous from $X$ with its $d$-Scott topology to $Y$ with its $\partial$-Scott topology [10, Proposition 2.5].

Given any topological space $X$, a map $h: X \rightarrow \overline{\mathbb{R}}_{+}$is lower semicontinuous if and only if it is continuous from $X$ to $\overline{\mathbb{R}}_{+}$with its Scott topology. We write $\mathcal{L} X$ for the space of all lower semicontinuous maps from $X$ to $\overline{\mathbb{R}}_{+}$, with the Scott topology of the pointwise ordering. For every open subset $U$ of $X$, the characteristic map $\chi_{U}$, which maps every element of $U$ to 1 and all other elements to 0 , is lower semicontinuous.

Given any standard quasi-metric space $X, d$, every $\alpha$-Lipschitz continuous 
map from $X, d$ to $\overline{\mathbb{R}}_{+}, \mathrm{d}_{\mathbb{R}}$ is lower semicontinuous. We write $\mathcal{L}_{\alpha}(X, d)$, or simply $\mathcal{L}_{\alpha} X$, for the subspace of $\mathcal{L} X$ consisting of all $\alpha$-Lipschitz continuous maps. Note that this is indeed a subset: every $\alpha$-Lipschitz continuous map from a standard quasi-metric space $X, d$ to $\overline{\mathbb{R}}_{+}$is lower semicontinuous; and this allows us to give $\mathcal{L}_{\alpha}(X, d)$ the subspace topology induced by the inclusion in $\mathcal{L} X$.

We also write $\mathcal{L}_{\infty}(X, d)$ or $\mathcal{L}_{\infty} X$ for the space of all Lipschitz continuous maps, namely $\bigcup_{\alpha \in \mathbb{R}_{+}} \mathcal{L}_{\alpha}(X, d)$, still with the subspace topology from $\mathcal{L} X$.

Given a map $h: X \rightarrow \overline{\mathbb{R}}_{+}$, where $X, d$ is standard, define $h^{\prime}: \mathbf{B}(X, d) \rightarrow$

$\mathbb{R} \cup\{+\infty\}$ by $h^{\prime}(x, r) \stackrel{\text { def }}{=} h(x)-\alpha r$. Then $h$ is $\alpha$-Lipschitz if and only if $h^{\prime}$ is monotonic, and $h$ is $\alpha$-Lipschitz continuous if and only if $h^{\prime}$ is Scottcontinuous [10, Lemma 2.7].

The Lipschitz continuous maps from a standard quasi-metric space to $\overline{\mathbb{R}}_{+}, \mathrm{d}_{\mathbb{R}}$ enjoy the usual closure properties [13, Proposition 6.7]: if $f$ is $\beta$ Lipschitz continuous then $\alpha f$ is $\alpha \beta$-Lipschitz continuous; if $f$ is $\alpha$-Lipschitz continuous and $g$ is $\beta$-Lipschitz continuous then $f+g$ is $(\alpha+\beta)$-Lipschitz continuous; if $f, g$ are $\alpha$-Lipschitz continuous, then so are $\min (f, g)$ and $\max (f, g)$; if $\left(f_{i}\right)_{i \in I}$ is any family of $\alpha$-Lipschitz continuous maps, then the pointwise supremum $\sup _{i \in I} f_{i}$ is also $\alpha$-Lipschitz continuous; if $\alpha \leq \beta$ and $f$ is $\alpha$-Lipschitz continuous then $f$ is $\beta$-Lipschitz continuous; every constant map is $\alpha$-Lipschitz continuous. Moreover, if $f: X \rightarrow Y$ is $\alpha$-Lipschitz continuous and $g: Y \rightarrow Z$ is $\beta$-Lipschitz continuous, then $g \circ f$ is $\alpha \beta$-Lipschitz continuous [10, Lemma 2.9].

Finally, for every map $f: X \rightarrow \overline{\mathbb{R}}_{+}$from a standard quasi-metric space $X, d$ to $\overline{\mathbb{R}}_{+}$, there is a largest $\alpha$-Lipschitz continuous map $f^{(\alpha)}$ below $f$. When $f$ is lower semicontinuous, the family $\left(f^{(\alpha)}\right)_{\alpha \in \mathbb{R}_{+}}$is a chain, and $\sup _{\alpha \in \mathbb{R}_{+}} f^{(\alpha)}=$ $f$, where suprema are taken pointwise [13, Theorem 6.17].

\section{Previsions and Hyperspaces}

We have introduced previsions in a somewhat loose fashion in the introduction. Let us fix the vocabulary. The linear previsions are in one-to-one correspondence with continuous valuations (i.e., measures, up to some details), as studied in Part I [12]; the normalized linear previsions correspond to probability continuous valuations, and the subnormalized linear previsions to subprobability continuous valuations. The sublinear and superlinear previsions will be the topic of Part III. In this paper, we will be particularly interested in the discrete sublinear (resp. superlinear) previsions. 
Definition 3.1 (Prevision). A prevision on a topological space $X$ is a Scottcontinuous map $F$ from $\mathcal{L} X$ to $\overline{\mathbb{R}}_{+}$that is positively homogeneous, namely: $F(\alpha h)=\alpha F(h)$ for all $\alpha \in \mathbb{R}_{+}, h \in \mathcal{L} X$.

It is:

- sublinear if $F\left(h+h^{\prime}\right) \leq F(h)+F\left(h^{\prime}\right)$ holds for all $h, h^{\prime} \in \mathcal{L} X$,

- superlinear if $F\left(h+h^{\prime}\right) \geq F(h)+F\left(h^{\prime}\right)$ holds,

- linear if it is both sublinear and superlinear,

- subnormalized if $F(\alpha . \mathbf{1}+h) \leq \alpha+F(h)$ holds, where $\mathbf{1}$ is the constant 1 map,

- normalized if $F(\alpha .1+h)=\alpha+F(h)$ holds,

- discrete if $F(f \circ h)=f(F(h))$ for every $h \in \mathcal{L} X$ and every strict $f \in \mathcal{L} \overline{\mathbb{R}}_{+}-a$ map $f$ is strict if and only if $f(0)=0$.

As for discreteness, we note that a map $f \in \mathcal{L} \overline{\mathbb{R}}_{+}$is, equivalently, a Scottcontinuous map from $\overline{\mathbb{R}}_{+}$to $\overline{\mathbb{R}}_{+}$. We write $\mathbf{P} X$ for the set of previsions on $X$.

\subsection{Closed sets and discrete sublinear previsions}

We first show that closed sets are related to discrete sublinear previsions. This is similar to Lemma 4.7, item 1, of [8].

Proposition 3.2. Let $X$ be a topological space. For each subset $C$ of $X$, let $F^{C}(h) \stackrel{\text { def }}{=} \sup _{x \in C} h(x)$. (We agree that this is equal to 0 if $C=\emptyset$.)

1. If $C$ is closed, then $F^{C}$ is a discrete sublinear prevision.

2. Conversely, every discrete sublinear prevision is of the form $F^{C}$ for some unique closed set $C$.

Moreover, $F$ is normalized if and only if $C$ is non-empty.

Proof. We start with the last claim, and show that $F^{C}$ is normalized if and only if $C$ is non-empty. If $C=\emptyset, F^{C}=0$ is not normalized. Otherwise, $F^{C}(\alpha .1+h)=\sup _{x \in C}(\alpha+h(x))=\alpha+\sup _{x \in C} h(x)=\alpha+F^{C}(h)$. 
1. $F^{C}$ is clearly a prevision. We check that $F^{C}$ is discrete. If $C=\emptyset$, then $F^{C}=0$ and for every strict $f \in \mathcal{L} \overline{\mathbb{R}}_{+}, F^{C}(f \circ h)=0=f\left(F^{C}(h)\right)-$ because $f$ is strict. Otherwise, $f\left(F^{C}(h)\right)=\sup _{x \in C} f(h(x))$ because every non-empty family in $\overline{\mathbb{R}}_{+}$is directed, and because $f$ is Scott-continuous. Since $\sup _{x \in C} f(h(x))=F^{C}(f \circ h)$, we conclude that $F^{C}$ is discrete.

Clearly, $F^{C}\left(h+h^{\prime}\right)=\sup _{x \in C}\left(h(x)+h^{\prime}(x)\right) \leq \sup _{x \in C} h(x)+\sup _{x \in C} h^{\prime}(x)$, so $F^{C}$ is sublinear.

2. Let now $F$ be an arbitrary discrete sublinear prevision on $X$. Let $\mathcal{F}$ be the family of open subsets $U$ of $X$ such that $F\left(\chi_{U}\right)=0 . \mathcal{F}$ contains the empty set, hence is non-empty. If $U \subseteq V$ and $V \in \mathcal{F}$, then $U$ is in $\mathcal{F}$ because $F$ is monotonic. For every directed family $\left(U_{i}\right)_{i \in I}$ of elements of $\mathcal{F}$, letting $U \stackrel{\text { def }}{=}$ $\bigcup_{i \in I} U_{i},\left(\chi_{U_{i}}\right)_{i \in I}$ is a directed family of lower semicontinuous maps whose supremum is $\chi_{U}$. Since $F$ is Scott-continuous, $F\left(\chi_{U}\right)=\sup _{i \in I} F\left(\chi_{U_{i}}\right)=0$, so $U$ is in $\mathcal{F}$. We have shown that $\mathcal{F}$ is Scott-closed.

We observe that $\mathcal{F}$ is directed. Take any two elements $U_{1}, U_{2}$ of $\mathcal{F}$. Since $F$ is sublinear, $F\left(\chi_{U_{1}}\right)+F\left(\chi_{U_{2}}\right) \geq F\left(\chi_{U_{1}}+\chi_{U_{2}}\right)=F\left(\chi_{U_{1} \cup U_{2}}+\chi_{U_{1} \cap U_{2}}\right) \geq$ $F\left(\chi_{U_{1} \cup U_{2}}\right)$, so the latter is equal to 0 , meaning that $U_{1} \cup U_{2}$ is in $\mathcal{F}$.

$\mathcal{F}$ being Scott-closed and directed, it has a largest element $U_{0}$, and therefore $\mathcal{F}$ is the set of open subsets of $U_{0}$.

We can now prove item 2. If $F=F^{C}$, then $\mathcal{F}$ is the family of open subsets that do not intersect $C$, so $U_{0}$ is the complement of $C$. This shows the uniqueness of $C$.

As far as existence is concerned, we are led to define $C$ as the complement of $U_{0}$.

We check that $F=F^{C}$. Let $h$ be a lower semicontinuous map from $X$ to $\overline{\mathbb{R}}_{+}$. For every $t \in \mathbb{R}_{+}$, let $f \stackrel{\text { def }}{=} \chi_{] t,+\infty]}$ and notice that this is a strict lower semicontinuous map. Since $F$ is discrete, $F(f \circ h)=f(F(h))$. Noting that $f \circ h=\chi_{U}$ where $U$ is the open set $\left.\left.h^{-1}(] t,+\infty\right]\right), U \in \mathcal{F}$ is equivalent to $f(F(h))=0$. In other words, $\left.\left.h^{-1}(] t,+\infty\right]\right) \subseteq U_{0}$ if and only if $F(h) \leq t$. We notice that $F^{C}(h) \leq t$ if and only if $h(x) \leq t$ for every $x \in C$, if and only if every $x \in X$ such that $h(x)>t$ is in $U_{0}$ (by contraposition), if and only if $\left.\left.h^{-1}(] t,+\infty\right]\right) \subseteq U_{0}$. Therefore $F^{C}(h) \leq t$ if and only if $F(h) \leq t$, for every $t \in \mathbb{R}_{+}$. It follows that $F^{C}(h)=F(h)$.

The Hoare powerdomain $\mathcal{H} X$ on a topological space is usually defined as the dcpo of all non-empty closed subsets of $X$, ordered by inclusion [5, Section IV-8]. We will also consider $\mathcal{H}_{0} X$, the dcpo of all, possibly empty, closed subsets of $X$. The bijection of Proposition 3.2 is an order-isomorphism 
between $\mathcal{H}_{0} X$ and the set of discrete sublinear previsions on $X$, ordered by the pointwise ordering.

One can also consider $\mathcal{H}_{0} X$, resp. $\mathcal{H} X$, as a space with the so-called lower Vietoris topology. In that case, we will speak of the Hoare hyperspace. That topology is generated by subbasic open sets $\diamond U \stackrel{\text { def }}{=}\{C \mid C \cap U \neq \emptyset\}$, where $U$ ranges over the open subsets of $X$. We will need the following definition, inspired from a similar definition on the probabilistic powerdomain [12, Section 9].

Definition 3.3 (Weak topology). The weak topology on any space $Y$ of previsions on a topological space $X$ is the coarsest topology such that $F \mapsto$ $F(h)$ is lower semicontinuous for every $h \in \mathcal{L} X$.

In other words, the weak topology has subbasic open sets of the form $[h>a]$, defined as the set of previsions $F \in Y$ such that $F(h)>a$, for each $h \in \mathcal{L} X$ and $a \in \mathbb{R}_{+}$.

Lemma 3.4. Let $X$ be a topological space. The map $C \mapsto F^{C}$ is a homeomorphism of $\mathcal{H}_{0} X$ (resp., $\mathcal{H} X$ ) with the lower Vietoris topology onto the space of discrete sublinear previsions on $X$ (resp., that are normalized) with the weak topology.

Proof. This is a bijection by Proposition 3.2. Fix $h \in \mathcal{L} X, a \in \mathbb{R}_{+}$. For every $C \in \mathcal{H}_{0} X, F^{C}$ is in $[h>a]$ if and only if $\sup _{x \in C} h(x)>a$, if and only if $h(x)>a$ for some $x \in C$, if and only if $C$ intersects $\left.\left.h^{-1}(] a,+\infty\right]\right)$, namely, $\left.\left.C \in \diamond h^{-1}(] a,+\infty\right]\right)$. Therefore the bijection is continuous.

In the other direction, for every open subset $U, \diamond U$ is the set of all $C \in \mathcal{H}_{0} X$ such that $C$ intersects $\left.\chi_{U}^{-1}(11 / 2,+\infty]\right)$, i.e., such that $F^{C}$ is in $\left[\chi_{U}>1 / 2\right]$. The case of $\mathcal{H} X$ is similar.

\subsection{Compact saturated sets and discrete superlinear previsions}

Dually to the Hoare hyperspace, we have the Smyth hyperspace. The following proposition is inspired from [8, Lemma 4.7, item 2]. For the notion of sober space, see [7, Chapter 8]. We will use the Hofmann-Mislove theorem: every Scott-open filter of open subsets of a sober space is the filter of open neighborhoods of a unique compact saturated subset $Q$ [7, Theorem 8.3.2]. All Hausdorff spaces are sober, all continuous dcpos are sober in their Scott topology [7, Proposition 8.2.12]; more centrally to the themes of this paper, all continuous complete quasi-metric spaces, in particular all 
algebraic complete quasi-metric spaces, are sober in their $d$-Scott topology [13, Proposition 4.1].

Proposition 3.5. Let $X$ be a topological space. For each non-empty subset $Q$ of $X$, let $F_{Q}(h) \stackrel{\text { def }}{=} \inf _{x \in Q} h(x)$.

1. If $Q$ is compact saturated and non-empty, then $F_{Q}$ is a normalized discrete superlinear prevision; moreover, the infimum defining $F_{Q}$ is attained, namely $F_{Q}(h)=\min _{x \in Q} h(x)$ for every $h \in \mathcal{L} X$.

2. Conversely, if $X$ is sober, then every normalized discrete superlinear prevision is of the form $F_{Q}$ for some unique non-empty compact saturated set $Q$.

Proof. 1. For every $h \in \mathcal{L} X,\{h(x) \mid x \in Q\}$ is compact in $\overline{\mathbb{R}}_{+}$with its Scott topology, and non-empty, hence has a least element. It follows that there is an $x \in Q$ such that $h(x)=F_{Q}(h)$. This justifies the second subclaim, that $F_{Q}(h)=\min _{x \in Q} h(x)$.

$F_{Q}$ is clearly positively homogeneous and monotonic. To show Scottcontinuity, let $h$ be written as the supremum of a directed family $\left(h_{i}\right)_{i \in I}$ in $\mathcal{L} X . F_{Q}(h) \geq \sup _{i \in I} F_{Q}\left(h_{i}\right)$ is a consequence of monotonicity. If the inequality were strict, then there would be a number $r \in \mathbb{R}_{+}$such that $F_{Q}(h)>$ $r \geq \sup _{i \in I} F_{Q}\left(h_{i}\right)$. The inequality $F_{Q}(h)>r$, equivalently $\min _{x \in Q} h(x)>r$, means that the image of $Q$ by $h$ is contained in the Scott-open $] r,+\infty]$. For every $x \in Q, h(x)=\sup _{i \in I} h_{i}(x)>r$, so $h_{i}(x)>r$ for some $i \in I$. It follows that $Q$ is included in the union of the open subsets $\left.\left.h_{i}^{-1}(] r,+\infty\right]\right)$. Since $Q$ is compact and the family of the latter open subsets is directed, $Q$ is included in one of them, say $\left.\left.h_{i}^{-1}(] r,+\infty\right]\right)$. That means that the image of $Q$ by $h_{i}$ is included in $] r,+\infty]$, and that implies that $F_{Q}\left(h_{i}\right)=\min _{x \in Q} h_{i}(x)>r$, contradicting $r \geq \sup _{i \in I} F_{Q}\left(h_{i}\right)$.

We check that $F_{Q}$ is discrete. Let $x \in Q$ be such that $h(x)=F_{Q}(h)$. For every $f \in \mathcal{L} \overline{\mathbb{R}}_{+}$(strict or not), $F_{Q}(f \circ h)=\min _{x^{\prime} \in Q} f\left(h\left(x^{\prime}\right)\right)$ is trivially less than or equal to $f(h(x))$. For every $x^{\prime} \in Q, h\left(x^{\prime}\right) \geq h(x)$, so $(f \circ h)\left(x^{\prime}\right) \geq$ $f(h(x))$, from which it follows that $F_{Q}(f \circ h)=\min _{x^{\prime} \in Q}(f \circ h)\left(x^{\prime}\right) \geq f(h(x))=$ $f\left(F_{Q}(h)\right)$.

Since this holds even when $f$ is not strict, it holds for the map $f(x) \stackrel{\text { def }}{=}$ $\alpha+x$, for any $\alpha \in \mathbb{R}_{+}$. In other words, $F_{Q}(\alpha .1+h)=\alpha+F_{Q}(h)$, showing that $F_{Q}$ is normalized. 
Finally, we show that $F_{Q}$ is superlinear. Fix $h, h^{\prime} \in \mathcal{L} X$. Then $F_{Q}(h+$ $\left.h^{\prime}\right)=\min _{x \in Q}\left(h(x)+h\left(x^{\prime}\right)\right) \geq \min _{x \in Q} h(x)+\min _{x \in Q^{\prime}} h^{\prime}(x)=F_{Q}(h)+F_{Q}\left(h^{\prime}\right)$.

2. We now assume that $X$ is sober, and we let $F$ be a normalized discrete superlinear prevision on $X$. Let $\mathcal{F}$ be the family of open subsets $U$ of $X$ such that $F\left(\chi_{U}\right)=1 . \mathcal{F}$ is upwards-closed. Since $F$ is normalized, $\mathcal{F}$ contains $X$ itself, hence is non-empty.

Note that, for every open subset $U$ of $X, F\left(\chi_{U}\right)$ is either equal to 1 or to 0 . This is because $F$ is discrete: for every $t \in] 0,1], F\left(\chi_{U}\right)=F\left(\chi_{] t,+\infty]} \circ \chi_{U}\right)=$ $\chi_{] t,+\infty]}\left(F\left(\chi_{U}\right)\right) \in\{0,1\}$.

Using discreteness again and superlinearity, we show that, if $U \subseteq V$, then $F\left(\chi_{U}\right)+F\left(\chi_{V}\right)=F\left(\chi_{U}+\chi_{V}\right)$. The right-hand side is larger than or equal to the left-hand side by superlinearity. Let us imagine that it is strictly larger. Let $f \in \mathcal{L} \overline{\mathbb{R}}_{+}$map any $t \leq 1 / 2$ to 0 , any $\left.\left.t \in\right] 1 / 2,3 / 2\right]$ to 1 , and any $t>3 / 2$ to 2. By discreteness, $F\left(\chi_{U}+\chi_{V}\right)=F\left(f \circ\left(\chi_{U}+\chi_{V}\right)\right)=f\left(F\left(\chi_{U}+\chi_{V}\right)\right)$, which shows that $F\left(\chi_{U}+\chi_{V}\right) \in\{0,1,2\}$. Since $F\left(\chi_{U}\right)+F\left(\chi_{V}\right)<F\left(\chi_{U}+\chi_{V}\right)$, $F\left(\chi_{U}\right)$ and $F\left(\chi_{V}\right)$ cannot both be equal to 1 , and since $F\left(\chi_{U}\right) \leq F\left(\chi_{V}\right)$, at least $F\left(\chi_{U}\right)$ is equal to 0 . Hence $F\left(\chi_{V}\right)<F\left(\chi_{U}+\chi_{V}\right)$. Since $F\left(\chi_{U}+\right.$ $\left.\chi_{V}\right) \leq F\left(2 \chi_{V}\right)$, we obtain that $F\left(\chi_{U}+\chi_{V}\right)$ is a number in $\{0,1,2\}$ between $F\left(\chi_{V}\right)$ (exclusive) and $2 F\left(\chi_{V}\right)$ (inclusive). This implies that $F\left(\chi_{V}\right)=1$ and $F\left(\chi_{U}+\chi_{V}\right)=2$. We now let $f \stackrel{\text { def }}{=} \chi_{[3 / 2,+\infty]}$, and we observe that $f \circ\left(\chi_{U}+\chi_{V}\right)=\chi_{U}$. Therefore $F\left(f \circ\left(\chi_{U}+\chi_{V}\right)\right)=F\left(\chi_{U}\right)=0$. Вy discreteness, $F\left(f \circ\left(\chi_{U}+\chi_{V}\right)\right)=f\left(F\left(\chi_{U}+\chi_{V}\right)\right)=f(2)=1$, contradiction.

Using that, we show that $\mathcal{F}$ is a filter of open sets. The only thing that remains to be shown is that for any two elements $U_{1}, U_{2}$ of $\mathcal{F}, U_{1} \cap U_{2}$ is also in $\mathcal{F}$. Since $U_{1} \cap U_{2} \subseteq U_{1} \cup U_{2}$, by the equality we have just shown, $F\left(\chi_{U_{1} \cup U_{2}}\right)+F\left(\chi_{U_{1} \cap U_{2}}\right)=F\left(\chi_{U_{1} \cup U_{2}}+\chi_{U_{1} \cap U_{2}}\right)=F\left(\chi_{U_{1}}+\chi_{U_{2}}\right)$, which is larger than or equal to $F\left(\chi_{U_{1}}\right)+F\left(\chi_{U_{2}}\right)$ by superlinearity, that is, to 2 . Hence $F\left(\chi_{U_{1} \cap U_{2}}\right) \geq 2-F\left(\chi_{U_{1} \cup U_{2}}\right) \geq 1$, where the latter inequality comes from the fact that $F\left(\chi_{U_{1} \cup U_{2}}\right)$ can only be equal to 0 or to 1 . Since $F\left(\chi_{U_{1} \cap U_{2}}\right)$ can only be equal to 0 or to 1 , its value is 1 , so $U_{1} \cap U_{2}$ is in $\mathcal{F}$.

Finally, $\mathcal{F}$ is Scott-open, meaning that for every directed family $\left(U_{i}\right)_{i \in I}$ of open subsets whose union is in $\mathcal{F}$, some $U_{i}$ is in $\mathcal{F}$. This follows from the Scott-continuity of $F$. By the Hofmann-Mislove Theorem, $\mathcal{F}$ is the filter of open neighborhoods of a unique compact saturated subset $Q$. Then, for every open subset $U, Q \subseteq U$ if and only if $U \in \mathcal{F}$ if and only if $F\left(\chi_{U}\right)=1$. Since $F(0)=0$, the empty set is not in $\mathcal{F}$, so $Q$ is non-empty.

We check that $F=F_{Q}$. Let $h \in \mathcal{L} X$. For every $t \in \mathbb{R}_{+}$, let $f \stackrel{\text { def }}{=} \chi_{] t,+\infty]}$. 
Since $F$ is discrete, $F(f \circ h)=f(F(h))$. Let $\left.\left.U \stackrel{\text { def }}{=} h^{-1}(] t,+\infty\right]\right)$, so that $f \circ h=\chi_{U}$. Then $F(h)>t$ if and only if $f(F(h))=1$, if and only if $F\left(\chi_{U}\right)=1$ if and only if $Q \subseteq U$. If $Q \subseteq U$, then $F_{Q}(h)=\min _{x \in Q} h(x)>t$, and conversely. Therefore $F(h)>t$ if and only if $F_{Q}(h)>t$, for all $h$ and $t$, whence $F=F_{Q}$.

Finally, uniqueness is obvious: if $F=F_{Q}$ for some non-empty compact saturated subset $Q$, then $Q$ must be such that for every open subset $U$, $Q \subseteq U$ if and only if $F\left(\chi_{U}\right)=1$.

The Smyth powerdomain $\mathcal{Q} X$ of $X$ is the set of all non-empty compact saturated subsets $Q$ of $X$, ordered by reverse inclusion [5, Section IV-8]. When $X$ is sober, the bijection $Q \mapsto F_{Q}$ is an order-isomorphism between $\mathcal{Q} X$ and the set of normalized discrete superlinear previsions on $X$ with the pointwise ordering.

As with the Hoare powerdomain, one can also consider $\mathcal{Q} X$ as a space with the so-called upper Vietoris topology, whose basic open sets are $\square U \stackrel{\text { def }}{=}$ $\{Q \mid Q \subseteq U\}$, where $U$ ranges over the open subsets of $X$. This is the Smyth hyperspace.

Lemma 3.6. Let $X$ be a sober space. The map $Q \mapsto F_{Q}$ is a homeomorphism of $\mathcal{Q} X$ with the upper Vietoris topology onto the space of normalized discrete superlinear previsions on $X$ with the weak topology.

Proof. This is a bijection by Proposition 3.5. Fix $h \in \mathcal{L} X, a \in \mathbb{R}_{+}$. For every $Q \in \mathcal{Q} X, F_{Q}$ is in $[h>a]$ if and only if $\min _{x \in Q} h(x)>a$, if and only if $h(x)>a$ for every $x \in Q$, if and only if $\left.\left.Q \in \square h^{-1}(] a,+\infty\right]\right)$. Therefore the bijection is continuous. In the other direction, for every open subset $U, \square U$ is the set of all $Q \in \mathcal{Q} X$ such that $F_{Q} \in\left[\chi_{U}>1 / 2\right]$.

\section{The Kantorovich-Rubinstein Quasi-Metrics on Spaces of Previ- sions}

Let $X, d$ be a quasi-metric space, with its $d$-Scott topology.

\subsection{The Unbounded Kantorovich-Rubinstein Quasi-Metric}

We repeat the definition of the introduction.

Definition $4.1\left(d_{\mathbf{K R}}\right)$. Let $X, d$ be a quasi-metric space. The KantorovichRubinstein quasi-metric on the space of previsions on $X$ is defined by:

$$
d_{K R}\left(F, F^{\prime}\right) \stackrel{\text { def }}{=} \sup _{h \in \mathcal{L}_{1} X} \mathrm{~d}_{\mathbb{R}}\left(F(h), F^{\prime}(h)\right)
$$


This is a quasi-metric on any standard quasi-metric space, and $F \leq F^{\prime}$ if and only if $d_{\mathrm{KR}}\left(F, F^{\prime}\right)=0$ [12, Lemma 3.7].

Lemma 3.10 of $[12]$ characterizes the ordering on spaces $\mathbf{B}\left(Y, d_{\mathrm{KR}}\right)$, where $Y$ is any space of previsions on $X$ as follows.

Lemma 4.2. Let $X, d$ be a standard quasi-metric space, let $F, F^{\prime}$ be two previsions on $X$, and let $r, r^{\prime}$ be two elements of $\mathbb{R}_{+}$. The following are equivalent:

1. $(F, r) \leq d_{K R}^{+}\left(F^{\prime}, r^{\prime}\right)$;

2. $r \geq r^{\prime}$ and, for every $h \in \mathcal{L}_{1}(X, d), F(h)-r \leq F^{\prime}(h)-r^{\prime}$;

3. $r \geq r^{\prime}$ and, for every $h \in \mathcal{L}_{\alpha}(X, d), \alpha>0, F(h)-\alpha r \leq F^{\prime}(h)-\alpha r^{\prime}$.

Recall that $\mathcal{L}_{\infty} X$ has the subspace topology from $\mathcal{L} X$. Let us introduce the following variant on the notion of prevision. We do this, because our completeness theorem will naturally produce $\mathcal{L}$-previsions, not previsions, as $d_{\mathrm{KR}}$-limits. Showing that every $\mathcal{L}$-prevision defines a unique prevision will be the subject of Proposition 4.5.

Definition 4.3 (L-prevision). Let $X, d$ be a quasi-metric space. An $\mathcal{L}$ prevision on $X$ is any continuous map $G$ from $\mathcal{L}_{\infty}(X, d)$ to $\overline{\mathbb{R}}_{+}$such that $G(\alpha h)=\alpha G(h)$ for all $\alpha \in \mathbb{R}_{+}$and $h \in \mathcal{L}_{\infty}(X, d)$.

The notions of sublinearity, superlinearity, linearity, normalization, subnormalization, discreteness, carry over to $\mathcal{L}$-previsions, taking care to quantify over $h, h^{\prime} \in \mathcal{L}_{\infty} X$ and over $f$ strict in $\mathcal{L}_{\infty} \overline{\mathbb{R}}_{+}$. We write $\mathcal{L} \mathbf{P} X$ for the set of all $\mathcal{L}$-previsions on $X$, and equip it with a quasi-metric defined by the same formula as Definition 4.1, and which we denote by $d_{\mathrm{KR}}$ again.

Every $F \in \mathbf{P} X$ defines an element $F_{\mid \mathcal{L}_{\infty} X}$ of $\mathcal{L} \mathbf{P} X$ by restriction. Conversely, for every $G \in \mathcal{L} \mathbf{P} X$, let $\bar{G}(h) \stackrel{\text { def }}{=} \sup _{\alpha \in \mathbb{R}_{+}} G\left(h^{(\alpha)}\right)$. We will see that these two constructions are inverse of each other. We need the following lemma.

Lemma 4.4. Let $X, d$ be a standard quasi-metric space, $\alpha, \beta \in \mathbb{R}_{+}$. For all $h \in \mathcal{L} X$ and $f \in \mathcal{L} \overline{\mathbb{R}}_{+}, f^{(\alpha)} \circ h^{(\beta)} \leq(f \circ h)^{(\alpha \beta)}$.

Proof. $f^{(\alpha)} \circ h^{(\beta)}$ is $\alpha \beta$-Lipschitz continuous. For every $x \in X, f^{(\alpha)}\left(h^{(\beta)}(x)\right) \leq$ $f\left(h^{(\beta)}(x)\right) \leq f(h(x))$, so $f^{(\alpha)} \circ h^{(\beta)}$ is less than or equal to $f \circ h$, hence also to the largest $\alpha \beta$-Lipschitz continuous map below $f \circ h,(f \circ h)^{(\alpha \beta)}$. 
Proposition 4.5. Let $X, d$ be a standard quasi-metric space.

1. For every $G \in \mathcal{L} \mathbf{P} X, \bar{G}$ is a prevision.

2. The maps $G \in \mathcal{L} \mathbf{P} X \mapsto \bar{G} \in \mathbf{P} X$ and $F \in \mathbf{P} X \mapsto F_{\mid \mathcal{L}_{\infty} X} \in \mathcal{L} \mathbf{P} X$ are inverse of each other.

3. If $G$ is sublinear, resp. superlinear, resp. linear, resp. subnormalized, resp. normalized, resp. discrete, then so is $\bar{G}$.

4. Conversely, if $\bar{G}$ is sublinear, resp. superlinear, resp. linear, resp. subnormalized, resp. normalized, resp. discrete, then so is $G$.

Proof. This is Proposition 3.13 of [12], except for the preservation of discreteness in items 3 and 4. Accordingly, we only deal with those two points.

4. This is obvious, since by item $2, G$ is the restriction of $\bar{G}$ to $\mathcal{L}_{\infty}(X, d)$, and since every strict map in $\mathcal{L}_{\infty}(X, d)$ is also a strict map in $\mathcal{L} X$.

3. Let $G$ be a discrete prevision on $X$. We fix $h \in \mathcal{L} X$, and we let $f \in \mathcal{L} \overline{\mathbb{R}}_{+}$be strict. Note that for every $\beta \in \mathbb{R}_{+}, f^{(\beta)}$ is strict, too, since $0 \leq f^{(\beta)} \leq f$

We have $\bar{G}(f \circ h)=\sup _{\alpha \in \mathbb{R}_{+}} G\left((f \circ h)^{(\alpha)}\right)$. The map $f^{(\sqrt{\alpha})} \circ h^{(\sqrt{\alpha})}$ is $\alpha$-Lipschitz continuous and below $(f \circ h)^{(\alpha)}$ by Lemma 4.4. Therefore $\bar{G}(f \circ$ $h) \geq \sup _{\alpha \in \mathbb{R}_{+}} G\left(f^{(\sqrt{\alpha})} \circ h^{(\sqrt{\alpha})}\right)$. For all $\beta, \gamma$ in $\mathbb{R}_{+}, \beta$ and $\gamma$ are less than or equal to $\sqrt{\alpha}$ for $\alpha$ sufficiently large, so $\bar{G}(f \circ h) \geq \sup _{\beta, \gamma \in \mathbb{R}_{+}} G\left(f^{(\beta)} \circ\right.$ $\left.h^{(\gamma)}\right)=\sup _{\gamma \in \mathbb{R}_{+}} \sup _{\beta \in \mathbb{R}_{+}} f^{(\beta)}\left(G\left(h^{(\gamma)}\right)\right)$ (using the fact that $G$ is discrete) $=$ $\sup _{\gamma \in \mathbb{R}_{+}} f\left(G\left(h^{(\gamma)}\right)\right)=f\left(\sup _{\gamma \in \mathbb{R}_{+}} G\left(h^{(\gamma)}\right)\right.$ ) (since $f$ is lower semicontinuous from $\overline{\mathbb{R}}_{+}$to itself, hence Scott-continuous $)=f(\bar{G}(h))$.

Conversely, $f \circ h$ is the supremum of the directed family $\left(f^{(\beta)} \circ h^{(\gamma)}\right)_{\beta, \gamma \in \mathbb{R}_{+}}$. This is directed because both $\left(f^{(\beta)}\right)_{\beta \in \mathbb{R}_{+}}$and $\left(h^{(\gamma)}\right)_{\gamma \in \mathbb{R}_{+}}$are chains, and every $f^{(\beta)}$ is monotonic (remember that every $\beta$-Lipschitz continuous map is lower semicontinuous, and that every lower semicontinuous map is monotonic). Moreover, for every $x \in X, \sup _{\beta, \gamma \in \mathbb{R}_{+}} f^{(\beta)}\left(h^{(\gamma)}(x)\right)=\sup _{\gamma \in \mathbb{R}_{+}} \sup _{\beta \in \mathbb{R}_{+}} f^{(\beta)}\left(h^{(\gamma)}(x)\right)=$ $\sup _{\gamma \in \mathbb{R}_{+}} f\left(h^{(\gamma)}(x)\right)=f\left(\sup _{\gamma \in \mathbb{R}_{+}} h^{(\gamma)}(x)\right)=f(h(x))$.

Using that, we show that $\bar{G}(f \circ h) \leq f(\bar{G}(h))$, from which the equality will follow. For every $a<\bar{G}(f \circ h)$, since $\bar{G}$ is Scott-continuous by item 1 , and using the previous observation, there are $\beta, \gamma \in \mathbb{R}_{+}$such that $a<$ $\bar{G}\left(f^{(\beta)} \circ h^{(\gamma)}\right)$. Since $f^{(\beta)} \circ h^{(\gamma)}$ is in $\mathcal{L}_{\beta \gamma}(X, d), \bar{G}\left(f^{(\beta)} \circ h^{(\gamma)}\right)=G\left(f^{(\beta)} \circ h^{(\gamma)}\right)$. Since $G$ is discrete, the latter is equal to $f^{(\beta)}\left(G\left(h^{(\gamma)}\right)\right)$, which is less than or 
equal to $f\left(G\left(h^{(\gamma)}\right)\right.$ ), hence to $f(\bar{G}(h))$ (recall that $f$ is lower semicontinuous hence monotonic). Since $a$ is arbitrary, $\bar{G}(f \circ h) \leq f(\bar{G}(h))$.

We can equip $\mathcal{L} \mathbf{P} X$ with a quasi-metric defined by formula (1), which we will again denote by $d_{\mathrm{KR}}$. Recall that an isometry is a map that preserves distances on the nose. The map $F \in \mathbf{P} X \mapsto F_{\mid \mathcal{L}_{\infty} X} \in \mathcal{L} \mathbf{P} X$ is an isometry, by definition.

If two maps $f: X, d \mapsto Y, \partial$ and $g: Y, \partial \rightarrow X, d$ are mutually inverse isometries, then they are not only 1-Lipschitz but also 1-Lipschitz continuous. Indeed, $\mathbf{B}^{1}(f)$ and $\mathbf{B}^{1}(g)$ are order-isomorphisms, and therefore are both Scott-continuous. (This is a standard exercise.)

It follows that, for the purpose of quasi-metrics, of the underlying $d$-Scott topologies, and for the underlying specialization orderings, $\mathcal{L} \mathbf{P} X$ and $\mathbf{P} X$ can be regarded as the same space under $d_{\mathrm{KR}}$.

Next, we need the following lemma.

Lemma 4.6. Every function $f \in \mathcal{L}_{\infty} \overline{\mathbb{R}}_{+}$is continuous from $\overline{\mathbb{R}}_{+}$to $\overline{\mathbb{R}}_{+}$, with its usual Hausdorff topology.

Proof. Let $f \in \mathcal{L}_{\beta} \overline{\mathbb{R}}_{+}, \beta>0$. The Hausdorff topology on $\overline{\mathbb{R}}_{+}$is generated by the Scott-open subsets $] a,+\infty]$ and the subsets of the form $[0, b[$. Since $\mathcal{L}_{\beta} \overline{\mathbb{R}}_{+} \subseteq \mathcal{L} \overline{\mathbb{R}}_{+}$(owing to the fact that $\overline{\mathbb{R}}_{+}, \mathrm{d}_{\mathbb{R}}$ is standard), $\left.f^{-1}(] a,+\infty\right]$ ) is Scott-open hence open in $\overline{\mathbb{R}}_{+}$. And $f^{-1}([0, b[)$ is open because $f$ is $\beta$ Lipschitz: for every $x \in f^{-1}([0, b[)$, let $\epsilon>0$ be such that $f(x)+\epsilon<b$; for every $x^{\prime} \in\left[0, x+\epsilon / \beta\left[, f\left(x^{\prime}\right) \leq f(x)+\beta \mathrm{d}_{\mathbb{R}}\left(x^{\prime}, x\right)<f(x)+\epsilon<b\right.\right.$, so $[0, x+\epsilon / \beta[$ is an open neighborhood of $x$ contained in $f^{-1}([0, b[)$.

The following proposition almost shows that our spaces of previsions are complete, and gives a simple formula for the supremum $(G, r)$ of a directed family of formal balls $\left(G_{i}, r_{i}\right)_{i \in I}$. I say "almost" because $G$ is not guaranteed to be continuous. We address that problem in the subsequent Theorem 4.8.

Proposition 4.7 (Naive suprema, $d_{\mathbf{K R}}$ case). Let $X, d$ be a standard quasimetric space, and $\left(G_{i}, r_{i}\right)_{i \in I}$ be a directed family in $\mathbf{B}\left(\mathcal{L} \mathbf{P} X, d_{K R}\right)$. Let $r \stackrel{\text { def }}{=}$ $\inf _{i \in I} r_{i}$ and, for each $h \in \mathcal{L}_{\alpha}(X, d), \alpha>0$, define $G(h)$ as the directed supremum $\sup _{i \in I}\left(G_{i}(h)+\alpha r-\alpha r_{i}\right)$. Then:

1. $G$ is a well-defined, positively homogeneous function from $\mathcal{L}_{\infty} X$ to $\overline{\mathbb{R}}_{+}$;

2. for any upper bound $\left(G^{\prime}, r^{\prime}\right)$ of $\left(G_{i}, r_{i}\right)_{i \in I}, r^{\prime} \leq r$ and, for every $h \in$ $\mathcal{L}_{\alpha}(X, d)$ with $\alpha>0, G(h) \leq G^{\prime}(h)+\alpha r-\alpha r^{\prime} ;$ 
3. if $G$ is continuous, then $(G, r)$ is the supremum of $\left(G_{i}, r_{i}\right)_{i \in I}$;

4. if every $G_{i}$ is sublinear, resp. superlinear, resp. linear, resp. subnormalized, resp. normalized, resp. discrete, then so is $G$.

We call $(G, r)$ the naive supremum of $\left(G_{i}, r_{i}\right)_{i \in I}$. By abuse of language, we will also call $G$ itself the naive supremum of $\left(G_{i}, r_{i}\right)_{i \in I}$.

Proof. This is Proposition 4.2 of [12] (or rather, the part about $d_{\mathrm{KR}}$ ), except for discreteness in item 4, which we deal with here. In the proof of that proposition, we noted that $G$ is well-defined, although the definition looks ambiguous, because $G(h)$ is in fact the limit of the net $\left(G_{i}(h)\right)_{i \in I}$ in $\overline{\mathbb{R}}_{+}$ with its usual Hausdorff (not Scott) topology, for every $h \in \bigcup_{\alpha>0} \mathcal{L}_{\alpha}(X, d)$. For every strict map $f \in \mathcal{L}_{\infty} \overline{\mathbb{R}}_{+}$, we then have $f(G(h))=f\left(\lim _{i \in I} G_{i}(h)\right)=$ $\lim _{i \in I} f\left(G_{i}(h)\right)$ by Lemma 4.6, and this is equal to $\lim _{i \in I} G_{i}(f \circ h)=G(f \circ h)$.

The following is not quite the completeness result we are after, because of the awkward assumption that the topology of $\mathcal{L}_{\infty}(X, d)$ is determined by those of $\mathcal{L}_{\alpha}(X, d), \alpha>0$, or equivalently that: any subset $U$ of $\mathcal{L}_{\infty}(X, d)$ such that $U \cap \mathcal{L}_{\alpha}(X, d)$ is open in $\mathcal{L}_{\alpha}(X, d)$ for every $\alpha>0$ is open in $\mathcal{L}_{\infty}(X, d)$, or also that: every map $G: \mathcal{L}_{\infty}(X, d) \rightarrow Z$, where $Z$ is any topological space, whose restriction to $\mathcal{L}_{\alpha}(X, d)$ is continuous for every $\alpha>0$, is itself continuous.

Theorem 4.8 (Completeness). Let $X, d$ be a standard quasi-metric space, and assume that the topology of $\mathcal{L}_{\infty}(X, d)$ is determined by those of $\mathcal{L}_{\alpha}(X, d)$, $\alpha>0$.

Then $\mathbf{P} X, d_{K R}$ is complete, and all suprema of directed families of formal balls of previsions are naive suprema, as described in Proposition 4.7.

The same result holds for the subspace of previsions satisfying any given set of properties among: sublinearity, superlinearity, linearity, subnormalization, normalization, and discreteness.

Proof. We use the assumptions, notations and results of Proposition 4.7. It remains to show that the naive supremum $G$, as defined there, is continuous from $\mathcal{L}_{\infty}(X, d)$ to $\overline{\mathbb{R}}_{+}$. For that, by the assumption that the topology of $\mathcal{L}_{\infty}(X, d)$ is determined, it suffices to show that the restriction $G_{\mid \mathcal{L}_{\alpha} X}$ of $G$ is continuous from $\mathcal{L}_{\alpha}(X, d)$ to $\overline{\mathbb{R}}_{+}$, for any $\alpha>0$. Fix $a \in \mathbb{R}$. We must show that $\mathcal{U} \stackrel{\text { def }}{=}\left\{h \in \mathcal{L}_{\alpha}(X, d) \mid G(h)>a\right\}$ is open in $\mathcal{L}_{\alpha}(X, d)$. Using the 
definition of $G$, we write $\mathcal{U}$ as the set of maps $h \in \mathcal{L}_{\alpha}(X, d)$ such that $G_{i}(h)+$ $\alpha r-\alpha r_{i}>a$ for some $i \in I$. Therefore $\left.\left.\mathcal{U}=\bigcup_{i \in I} G_{i \mid \mathcal{L}_{\alpha} X}^{-1}(] a+\alpha r_{i}-\alpha r,+\infty\right]\right)$, which is open.

Remark 4.9. As in [12], our strategy for establishing more interesting completeness theorems will be to embed $X, d$ into a better behaved quasi-metric space $Y, \partial$. We let $i: X \rightarrow Y$ be the embedding, and we will observe later that every prevision $G$ on $X$ yields a prevision $\mathbf{P} i(G)$ on $Y$ by the formula $\mathbf{P} i(G)(k)=G(k \circ i)$. (For a linear prevision, equivalently a continuous valuation, this formula yields the so-called push-forward, or image measure of $G$ by i.) Our proof strategy will require the following two properties: (i) the topology of $\mathcal{L}_{\infty}(Y, \partial)$ is determined by those of $\mathcal{L}_{\alpha}(Y, \partial), \alpha>0$, so that Theorem 4.8 will apply on $Y, \partial$; (ii) the naive suprema $(G, r)$ computed in $\mathbf{B}(\mathbf{P} Y, \partial)$ of directed families $\left(\mathbf{P} i\left(F_{i}\right), r_{i}\right)_{i \in I}$ is such that $G=\mathbf{P} i(F)$ for some prevision $F$ on $X$, and then $(F, r)$ is the supremum of the directed family $\left(F_{i}, r_{i}\right)_{i \in I}$.

For $Y, \partial$, we will take $\mathbf{B}(X, d), d^{+}$: as shown in [10, Theorem 4.13], this is always a so-called Lipschitz regular quasi-metric space, and Proposition 8.2 of [11] states that for any Lipschitz regular quasi-metric space $Y, \partial$, the topology of $\mathcal{L}_{\infty}(Y, \partial)$ is determined by those of $\mathcal{L}_{\alpha}(Y, \partial), \alpha>0$. This will take care of (i). The case of property (ii) will require specific work.

\subsection{The bounded Kantorovich-Rubinstein quasi-metrics}

In [12, Definition 3.4], we also introduced a so-called a-bounded form $d_{\mathrm{KR}}^{a}$ of $d_{\mathrm{KR}}$. The point was that, given a continuous complete quasi-metric space $X, d$ (and even a complete metric space $X, d$ ) the weak topology on the space $\mathbf{V}_{1} X$ of probability continuous valuations on $X$ does not coincide with the $d_{\mathrm{KR}}$-Scott topology in general, but does coincide with the $d_{\mathrm{KR}}^{a}$-Scott topology. The $d_{\mathrm{KR}}^{a}$ variant also extends to previsions, as we will see below.

Such a bounded form $d_{\mathrm{KR}}^{a}$ will be less interesting than the unbounded form $d_{\mathrm{KR}}$ here, but we need to explore this variant nonetheless, because we will require it in Part III, and we only need little additional effort to deal with it now.

For every quasi-metric space $X, d$, for every $a \in \mathbb{R}_{+} \backslash\{0\}$, let $\mathcal{L}_{\alpha}^{a}(X, d)$, or $\mathcal{L}_{\alpha}^{a} X$, be the subspace of $\mathcal{L}_{\alpha} X$ of those $\alpha$-Lipschitz continuous maps from $X, d$ to $\overline{\mathbb{R}}_{+}$that take their values in $[0, \alpha a]$. 
Definition $4.10\left(d_{\mathbf{K R}}^{a}\right)$. Let $X, d$ be a quasi-metric space, and $a \in \mathbb{R}_{+} \backslash$ $\{0\}$. The a-bounded Kantorovich-Rubinstein quasi-metric on the space of previsions on $X$ is defined by:

$$
d_{K R}^{a}\left(F, F^{\prime}\right) \stackrel{\text { def }}{=} \sup _{h \in \mathcal{L}_{1}^{a} X} \mathrm{~d}_{\mathbb{R}}\left(F(h), F^{\prime}(h)\right)
$$

This is a quasi-metric on any standard quasi-metric space, and $F \leq F^{\prime}$ if and only if $d_{\mathrm{KR}}^{a}\left(F, F^{\prime}\right)=0[12$, Lemma 3.7].

In order to deal with $d_{\mathrm{KR}}^{a}$, we will need the following further refinement of an $\mathcal{L}$-prevision. We write $\mathcal{L}_{\infty}^{\mathrm{b}}(X, d)$ for the space of all bounded Lipschitz continuous maps from $X$ to $\overline{\mathbb{R}}_{+}$.

Definition 4.11 ( $\mathcal{L}^{\mathrm{b}}$-prevision). Let $X, d$ be a quasi-metric space. An $\mathcal{L}^{\mathrm{b}}$ prevision on $X$ is any continuous map $H$ from $\mathcal{L}_{\infty}^{\mathrm{b}} X$ to $\overline{\mathbb{R}}_{+}$such that $H(\beta h)=$ $\beta H(h)$ for all $\beta \in \mathbb{R}_{+}$and $h \in \mathcal{L}_{\infty}^{\mathrm{b}} X$.

Let $\mathcal{L}^{\mathrm{b}} \mathbf{P} X$ be the set of all $\mathcal{L}^{\mathrm{b}}$-previsions on $X$. We define linear, superlinear, sublinear, subnormalized, and normalized $\mathcal{L}^{\mathrm{b}}$-previsions in the usual way. Finally, we say that $H$ is discrete iff it satisfies $H(f \circ h)=f(H(h))$ for every strict map $f \in \mathcal{L}_{\infty}^{\mathrm{b}} \overline{\mathbb{R}}_{+}$(not $\mathcal{L}_{\infty} \overline{\mathbb{R}}_{+}$) and every $h \in \mathcal{L}_{\infty}^{\mathrm{b}}(X, d)$.

Every $G \in \mathcal{L} \mathbf{P} X$ defines an element $G_{\mid \mathcal{L}_{\infty}^{\mathrm{b}} X}$ of $\mathcal{L}^{\mathrm{b}} \mathbf{P} X$ by restriction. Conversely, for every $H \in \mathcal{L}^{\mathrm{b}} \mathbf{P} X$, let $\overline{\bar{H}}(h) \stackrel{\text { def }}{=} \sup _{\beta>0} H(\min (h, \beta))$ for every $h \in \mathcal{L}_{\infty}(X, d)$.

Lemma 4.12. Let $X, d$ be a standard quasi-metric space, and $a>0$.

1. For every $H \in \mathcal{L}^{\mathrm{b}} \mathbf{P} X, \overline{\bar{H}}$ is an $\mathcal{L}$-prevision.

2. The maps $G \in \mathcal{L} \mathbf{P} X \mapsto G_{\mid \mathcal{L}_{\infty}^{\mathrm{b}} X} \in \mathcal{L}^{\mathrm{b}} \mathbf{P} X$ and $H \in \mathcal{L}^{\mathrm{b}} \mathbf{P} X \mapsto \overline{\bar{H}} \in \mathcal{L} \mathbf{P} X$ are inverse of each other.

3. For every $G \in \mathcal{L} \mathbf{P} X, G$ is linear, resp. superlinear, resp. sublinear, resp. subnormalized, resp. normalized, resp. discrete, if and only if $G_{\mid \mathcal{L}_{\infty}^{\mathrm{b} X}} i s$.

Proof. This is Lemma 3.18 of [12], except for the preservation of discreteness in item 3 , on which we concentrate.

If $G \in \mathcal{L} \mathbf{P} X$ is discrete, then clearly $G_{\mid \mathcal{L}_{\infty}^{\mathrm{b}} X}$ is discrete, too. Conversely, let $H \in \mathcal{L}^{\mathrm{b}} \mathbf{P} X$ be discrete. In order to show that $\overline{\bar{H}}$ is discrete, let $f \in$ 
$\mathcal{L}_{\infty} \overline{\mathbb{R}}_{+}$be strict, $h \in \mathcal{L}_{\infty}(X, d)$, and let us show that $\overline{\bar{H}}(f \circ h)=f(\overline{\bar{H}}(h))$. The left-hand side, $\overline{\bar{H}}(f \circ h)$, is equal to $\sup _{\beta>0} H(\min (f \circ h, \beta))$, hence to $\sup _{\beta>0} H\left(f_{\beta} \circ h\right)$, where $f_{\beta} \stackrel{\text { def }}{=} \min (f, \beta)$. Since $f_{\beta}$ is Scott-continuous and $H$ is the restriction of a Scott-continuous map $G: \mathcal{L}(X, d) \rightarrow \overline{\mathbb{R}}_{+}$(by item 2), $\overline{\bar{H}}(f \circ h)$ is also equal to $\sup _{\beta, \gamma>0} H\left(f_{\beta} \circ \min (h, \gamma)\right)$. We know that $\min (h, \gamma)$ is in $\mathcal{L}_{\infty}^{\mathrm{b}}(X, d)$, and that $f_{\beta}$ is in $\mathcal{L}_{\infty}^{\mathrm{b}} \overline{\mathbb{R}}_{+}$. Since $H$ is discrete, $\overline{\bar{H}}(f \circ h)$ is therefore equal to $\sup _{\beta, \gamma>0} f_{\beta}(H(\min (h, \gamma)))=\sup _{\gamma>0} f(H(\min (h, \gamma)))$. Now $f$ is Scott-continuous, so this is equal to $f\left(\sup _{\gamma>0} H(\min (h, \gamma))\right)=f(\overline{\bar{H}}(h))$.

The following is part of [12, Lemma 3.10].

Lemma 4.13. Let $X, d$ be a standard quasi-metric space, a $>0$, let $F, F^{\prime}$ be two previsions on $X$, and $r, r^{\prime}$ be two elements of $\mathbb{R}_{+}$. The following are equivalent:

1. $(F, r) \leq_{K R}^{a+}\left(F^{\prime}, r^{\prime}\right)$;

2. $r \geq r^{\prime}$ and, for every $h \in \mathcal{L}_{1}^{a}(X, d), F(h)-r \leq F^{\prime}(h)-r^{\prime}$;

3. $r \geq r^{\prime}$ and, for every $h \in \mathcal{L}_{\alpha}^{a}(X, d), \alpha>0, F(h)-\alpha r \leq F^{\prime}(h)-\alpha r^{\prime}$.

We can equip $\mathcal{L} \mathbf{P} X$ and $\mathcal{L}^{\mathrm{b}} \mathbf{P} X$ with a quasi-metric defined by formula (3), which we shall again denote by $d_{\mathrm{KR}}^{a}$. The maps $F \in \mathbf{P} X \mapsto F_{\mid \mathcal{L}_{\infty} X} \in \mathcal{L} \mathbf{P} X$ and $G \in \mathcal{L} \mathbf{P} X \mapsto G_{\mid \mathcal{L}_{\infty}^{\mathrm{b}} X} \in \mathcal{L}^{\mathrm{b}} \mathbf{P} X$ are isometries by definition.

Lemma 4.14. Let $X, d$ be a quasi-metric space. The maps:

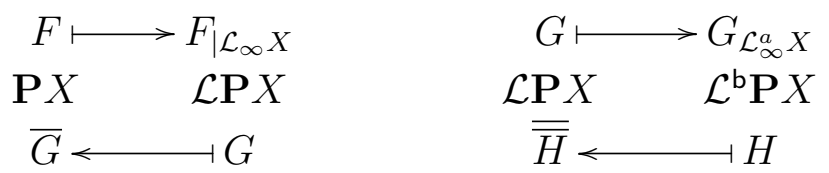

are isometries between $\mathbf{P} X, \mathcal{L} \mathbf{P} X$ and $\mathcal{L}^{\mathrm{b}} \mathbf{P} X$ with the $d_{K R}$ quasi-metrics, and with the $d_{K R}^{a}$ quasi-metrics, for every $a>0$.

It follows that, for the purpose of quasi-metrics, of the underlying $d$-Scott topologies, and for the underlying specialization orderings, $\mathcal{L} \mathbf{P} X, \mathcal{L}^{\mathrm{b}} \mathbf{P} X$, and $\mathbf{P} X$ can be regarded as the same space under $d_{\mathrm{KR}}$, or under $d_{\mathrm{KR}}^{a}$. 
Proposition 4.15 (Naive suprema, $d_{\mathbf{K R}}^{a}$ case). Fix $a>0$. Let $X, d$ be $a$ standard quasi-metric space, and $\left(H_{i}, r_{i}\right)_{i \in I}$ be a directed family in $\mathbf{B}\left(\mathcal{L}^{\mathrm{b}} \mathbf{P} X, d_{K R}^{a}\right)$. Let $r \stackrel{\text { def }}{=} \inf _{i \in I} r_{i}$ and, for each $h \in \mathcal{L}_{\alpha}^{a}(X, d), \alpha>0$, define $H(h)$ as $\sup _{i \in I}\left(H_{i}(h)+\alpha r-\alpha r_{i}\right)$. Then:

1. $H$ is a well-defined, positively homogeneous functional from $\mathcal{L}^{\mathrm{b}} \mathbf{P} X$ to $\overline{\mathbb{R}}_{+}$;

2. for any upper bound $\left(H^{\prime}, r^{\prime}\right)$ of $\left(H_{i}, r_{i}\right)_{i \in I}, r^{\prime} \leq r$ and, for every $h \in$ $\mathcal{L}_{\alpha}^{a}(X, d), \alpha>0, H(h) \leq H^{\prime}(h)+\alpha r-\alpha r^{\prime} ;$

3. if $H$ is continuous, then $(H, r)$ is the supremum of $\left(H_{i}, r_{i}\right)_{i \in I}$;

4. if every $H_{i}$ is sublinear, resp. superlinear, resp. linear, resp. subnormalized, resp. normalized, resp. discrete, then so is $H$.

Note that $H$ is indeed defined on the whole of $\mathcal{L}_{\infty}^{\mathrm{b}}(X, d)$, since $\mathcal{L}_{\infty}^{\mathrm{b}}(X, d)=$ $\bigcup_{\alpha>0} \mathcal{L}_{\alpha}^{a}(X, d)[11$, Lemma 3.5]. We again call $(H, r)$ the naive supremum of $\left(H_{i}, r_{i}\right)_{i \in I}$.

Proof. The proof is as for Proposition 4.7. Proposition 4.2 of [12] (the part on $d_{\mathrm{KR}}^{a}$, now) proves all the statements except those on discreteness; in item $4, H$ is discrete whenever every $H_{i}$ is, using Lemma 4.6, as before.

We deduce the following theorem with exactly the same proof as for Theorem 4.8, using Proposition 4.15 instead of Proposition 4.7.

Theorem 4.16 (Completeness). Let $X, d$ be a standard quasi-metric space, and fix $a>0$. Assume that the topology of $\mathcal{L}_{\infty}^{\mathrm{b}}(X, d)$ is determined by those of $\mathcal{L}_{\alpha}^{a}(X, d), \alpha>0$.

Then $\mathbf{P} X, d_{K R}^{a}$ is complete, and all suprema of directed families of formal balls of previsions are naive suprema, as described in Proposition 4.15.

The same result holds for the subspace of previsions satisfying any given set of properties among: sublinearity, superlinearity, linearity, subnormalization, normalization, and discreteness.

\subsection{Supports}

Our previous completeness theorems for spaces of previsions make a strong assumption, namely that the topology of $\mathcal{L}_{\infty}^{\mathrm{b}}(X, d)$ is determined by those of $\mathcal{L}_{\alpha}^{a}(X, d), \alpha>0$. We show that we also obtain completeness under different, and more natural, assumptions. 
Supports were crucial in Part I, and are essential here as well. The notion of a support of a measure is well-known, and it is not hard to generalize it to any prevision.

Definition 4.17 (Support). A subset $A$ of $X$ is a support of a prevision $G$ on a topological space $X$ if and only if for all $g, h \in \mathcal{L} X$ with the same restriction to $A$ (i.e., such that $g_{\mid A}=h_{\mid A}$ ), then $G(g)=G(h)$.

We also say that $G$ is supported on $A$ in that case.

Following Remark 4.9, we define $\mathbf{P} i(F)$ as mapping every $h \in \mathcal{L} X$ to $F(h \circ i)$, for any subspace inclusion $i$. This is a special case of a more general definition, which we will study in Lemma 4.21 .

Lemma 4.18. Let $X$ be a topological space. A subset $A$ of $X$ is a support of a prevision $G$ on $X$ if and only if there is a prevision $F$ on the subspace $A$ such that $G=\mathbf{P} i(F)$, where $i$ is the inclusion map of $A$ into $X$. If so, $F$ is unique, and $F(f)=G(\hat{f})$ for every $f \in \mathcal{L} A$, where $\hat{f}$ is the largest lower semicontinuous extension of $f$ to $X$.

Proof. If $G=\mathbf{P} i(F)$ for some prevision $F$ on $A$, then for all $g, h \in \mathcal{L} X$ with the same restriction to $A, g \circ i$ is equal to $h \circ i$, so $G(g)=F(g \circ i)=$ $F(h \circ i)=G(h)$.

Conversely, let us assume that $G$ is supported on $A$. We note that every map $f \in \mathcal{L} A$ extends to a lower semicontinuous map from $X$ to $\overline{\mathbb{R}}_{+}$. This is because $\overline{\mathbb{R}}_{+}$is a continuous complete lattice, hence an injective topological space [16]. Explicitly, there is even a largest lower semicontinuous extension $\hat{f}: X \rightarrow \overline{\mathbb{R}}_{+}$of $f$, and for every $x \in X, \hat{f}(x)=\sup _{U}$ inf $f[U \cap A]$, where $U$ ranges over the open neighborhoods of $x$ in $X$, and $f[U \cap A]$ denotes the image of $U \cap A$ by $f$ [5, Proposition II-3.9]; see also the proof of 2.11, page 109 , in [16].

For every $\alpha \in \mathbb{R}_{+}, \widehat{\alpha f}=\alpha \hat{f}$. Indeed, if $\alpha=0$, both sides are equal to the zero map. Otherwise, $\alpha \hat{f}$ is lower semicontinuous, below $\alpha f$, hence below the largest lower semicontinuous map $\widehat{\alpha f}$ below $\alpha f$. Replacing $\alpha$ by $1 / \alpha$ and $f$ by $\alpha f$, we obtain $1 / \alpha . \widehat{\alpha f} \leq \widehat{1 / \alpha \cdot \alpha f}=\hat{f}$, from which the reverse inequality $\widehat{\alpha f} \leq \hat{f}$ follows.

If there exists $F$ such that $G=\mathbf{P} i(F)$, then $G(\hat{f})=F(\hat{f} \circ i)=F(f)$ for every $f \in \mathcal{L} A$, so $F$ is unique.

For the existence part, we define $F: \mathcal{L} A \rightarrow \overline{\mathbb{R}}_{+}$by $F(f) \stackrel{\text { def }}{=} G(\hat{f})$. Since $\widehat{\alpha f}=\alpha \hat{f}, F$ is positively homogeneous. The map $f \mapsto \hat{f}$ is monotonic, so $F$ 
is monotonic as well. However, it is not Scott-continuous in general, namely unless $i$ is a finitary map, see [4, Theorem 2.7.2]. Hence we need a different argument to show that $F$ is Scott-continuous. Let $\left(f_{j}\right)_{j \in J}$ be a directed family with supremum $f$ in $\mathcal{L} A$. Let $g \stackrel{\text { def }}{=} \hat{f}$ and $h \stackrel{\text { def }}{=} \sup _{j \in J} \hat{f}_{j}$. We have $g \circ i=\hat{f} \circ i=f=\sup _{j \in J} f_{j}=\sup _{j \in J}\left(\hat{f}_{j} \circ i\right)=h \circ i$. Hence by assumption $G(g)=G(h)$. But $G(g)=G(\hat{f})=F(f)$, and $G(h)=G\left(\sup _{j \in J} \hat{f}_{j}\right)=$ $\sup _{j \in J} G\left(\hat{f}_{j}\right)=\sup _{j \in J} F\left(f_{j}\right)$, showing that $F$ is Scott-continuous.

Therefore $F$ is a prevision on $A$. Finally, for every $g \in \mathcal{L} X, \mathbf{P} i(F)(g)=$ $F(g \circ i)=G(\widehat{g \circ i})$. Let $h \stackrel{\text { def }}{=} \widehat{g \circ} i: h$ extends $g \circ i$, namely $h \circ i=g \circ i$, so $G(g)=G(h)$, by assumption. It follows that $\mathbf{P} i(F)(g)=G(g)$. Since $g$ is arbitrary, $\mathbf{P} i(F)=G$.

The situation simplifies when $A$ is an open subset $V$ of $X$.

Lemma 4.19. Let $X$ be a topological space. An open subset $V$ of $X$ is a support of a prevision $G$ on $X$ if and only if for every $g \in \mathcal{L} X, G(g)=$ $G\left(g \chi_{V}\right)$.

Proof. Clearly, $g$ and $g \chi_{V}$ have the same restriction to $V$. Hence if $V$ is a support of $G$, then $G(g)=G\left(g \chi_{V}\right)$. Conversely, if $G(g)=G\left(g \chi_{V}\right)$ for every $g \in \mathcal{L} X$, then for all $g, h \in \mathcal{L} X$ with the same restriction to $V, g \chi_{V}=h \chi_{V}$, so that $G(g)=G\left(g \chi_{V}\right)=G\left(h \chi_{V}\right)=G(h)$. Hence $V$ is a support of $G$.

Implementing the program of Remark 4.9, item (ii), we will define $(G, r)$ as the naive supremum of $\left(\mathbf{P} \eta_{X}\left(F_{i}\right), r_{i}\right)_{i \in I}$, where $\eta_{X}$ is the embedding $x \mapsto$ $(x, 0)$ of $X$ into $Y \stackrel{\text { def }}{=} \mathbf{B}(X, d)$. That means that:

$$
G(k)=\sup _{i \in I}\left(F_{i}\left(k \circ \eta_{X}\right)+\alpha r-\alpha r_{i}\right)
$$

for every $k \in \mathcal{L}_{\alpha}\left(\mathbf{B}(X, d), d^{+}\right), \alpha>0$ (resp., for every $k \in \mathcal{L}_{\alpha}^{a}\left(\mathbf{B}(X, d), d^{+}\right.$) if we are interested in $d_{\mathrm{KR}}^{a}$ rather than $\left.d_{\mathrm{KR}}\right)$. For that to make sense, we will need to study $\mathbf{P} \eta_{X}$, and we will do this in Section 4.4. For the time being, we note that $G(k)$, as defined by (4), only depends on $k \circ \eta_{X}$. In particular, this looks like saying that $G$ is supported on $X$, hence that $G=\mathbf{P} \eta_{X}(F)$ for some prevision $F$ on $X$, implementing (ii). That is not the case, because (4) is valid only for Lipschitz continuous maps $k$, and we need a statement that applies to all lower semicontinuous maps. Using Proposition 4.5 is not quite enough, and the best we can say is the following. We recall that $V_{\epsilon} \stackrel{\text { def }}{=}\{(x, r) \in \mathbf{B}(X, d) \mid r<\epsilon\}$ is Scott-open for every $\epsilon>0$, whenever $X, d$ 
is standard. It should be clear that the $\mathcal{L}$-prevision $G$ defined by (4) satisfies the assumptions of the following lemma.

Lemma 4.20. Let $X, d$ be a standard quasi-metric space. Let $G$ be an $\mathcal{L}$ prevision on $\mathbf{B}(X, d)$ that is supported on $X$ in the sense that for every $\alpha>0$, for all $f, g \in \mathcal{L}_{\alpha}(X, d)$ such that $f \circ \eta_{X}=g \circ \eta_{X}, G(f)=G(g)$. Then, for every $\epsilon>0, \bar{G}$ is supported on $V_{\epsilon}$.

Proof. We use Lemma 4.19. Let $g$ be any lower semicontinuous map from $\mathbf{B}(X, d)$ to $\overline{\mathbb{R}}_{+}$. We claim that $\bar{G}(g)=\bar{G}\left(g \chi_{V_{\epsilon}}\right)$. Since $g \chi_{V_{\epsilon}} \leq g$, we have $\bar{G}\left(g \chi_{V_{\epsilon}}\right) \leq \bar{G}(g)$. In the converse direction, we must show that $G\left(g^{(\alpha)}\right) \leq$ $\bar{G}\left(g \chi_{V_{\epsilon}}\right)$ for every $\alpha>0$.

Corollary 4.11 of [12] states that, given that $X, d$ is standard, for all $\beta \in \mathbb{R}_{+}$and $\epsilon>0$ such that $\beta \epsilon \geq 1, \chi_{V}^{(\beta)} \circ \eta_{X}=\chi_{V \cap V_{\epsilon}}^{(\beta)} \circ \eta_{X}$. Taking $V \stackrel{\text { def }}{=} \mathbf{B}(X, d)$, so that $\chi_{V}=\mathbf{1}$ and therefore $\chi_{V}^{(\beta)}=\mathbf{1}$, it follows that for every $\beta \geq 1 / \epsilon, \chi_{V_{\epsilon}}^{(\beta)} \circ \eta_{X}=\mathbf{1}$, and therefore $g^{(\alpha)} \circ \eta_{X}=\left(g^{(\alpha)} \chi_{V_{\epsilon}}^{(\beta)}\right) \circ \eta_{X}$.

We fix $\beta \geq 1 / \epsilon$, and also such that $\beta \geq 1$. Then $g^{(\alpha)}$ and $g^{(\alpha)} \chi_{V_{\epsilon}}^{(\beta)}$ are both $\alpha \beta$-Lipschitz continuous. By our assumption on $G$ (with $\alpha \beta$ instead of $\alpha), G\left(g^{(\alpha)}\right)=G\left(g^{(\alpha)} \chi_{V_{\epsilon}}^{(\beta)}\right)$.

Since $\bar{G}$ coincides with $G$ on Lipschitz continuous maps, $G\left(g^{(\alpha)}\right)=\bar{G}\left(g^{(\alpha)} \chi_{V_{\epsilon}}^{(\beta)}\right)$. The function $g^{(\alpha)} \chi_{V_{\epsilon}}^{(\beta)}$ is less than or equal to $g \chi_{V_{\epsilon}}$, so $G\left(g^{(\alpha)}\right) \leq \bar{G}\left(g \chi_{V_{\epsilon}}\right)$.

It is frustrating that we are not able to say that $G$ is supported on $X$ directly, although $X=\bigcap_{\epsilon>0} V_{\epsilon}$. In [12], we solved that problem for linear $\mathcal{L}$-previsions on continuous complete quasi-metric spaces by extending the associated continuous valuation to a measure. We will need different arguments in the case of each hyperspace, in Sections 5, and in 6 .

\subsection{The action of $\mathbf{P}$ on Lipschitz continuous maps}

We have already introduced the notation $\mathbf{P} i(F)$ for a prevision $F$ on a subset $A$ of a space $X$, where $i$ is the embedding of $A$ into $X$. This is a special case of the definition of a map $\mathbf{P} f: \mathbf{P} X \rightarrow \mathbf{P} Y$, for every continuous map $f: X \rightarrow Y$, as in (5) below. One may wonder whether this defines a functor on the category of standard quasi-metric spaces and 1-Lipschitz continuous maps. For now, we have the following partial result.

Lemma 4.21. Let $X, d$ and $Y, \partial$ be two quasi-metric spaces, and $f: X, d \mapsto$ $Y, \partial$ be an $\alpha$-Lipschitz continuous map, where $\alpha>0$. Then $\mathbf{P} f: \mathbf{P} X, d_{K R} \rightarrow$ 
$\mathbf{P} Y, \partial_{K R}$, defined by:

$$
\mathbf{P} f(F)(k) \stackrel{\text { def }}{=} F(k \circ f)
$$

for every $k \in \mathcal{L} Y$, is a well-defined, $\alpha$-Lipschitz map, which maps previsions to previsions, preserving sublinearity, superlinearity, linearity, subnormalization, normalization, and discreteness.

A similar result holds for $\mathbf{P} f$, seen as a map from $\mathbf{P} X, d_{K R}^{a}$ to $\mathbf{P} Y, \partial_{K R}^{a}$, for every $a \in \mathbb{R}_{+}, a>0$.

Proof. It is easy to check that $\mathbf{P} f(F)$ is a prevision, which is sublinear, resp. superlinear, resp. linear, resp. subnormalized, resp. normalized, resp. discrete, whenever $F$ is.

In order to show that $\mathbf{P} f$ is $\alpha$-Lipschitz from $\mathbf{P} X, d_{\mathrm{KR}}$ to $\mathbf{P} Y, \partial_{\mathrm{KR}}$, we assume that $d_{\mathrm{KR}}\left(F, F^{\prime}\right) \leq r$, and we wish to show that $\partial_{\mathrm{KR}}\left(\mathbf{P} f(F), \mathbf{P} f\left(F^{\prime}\right)\right) \leq$ $\alpha r$. To this end, we let $k \in \mathcal{L}_{1}\left(\mathbf{B}(Y, \partial), \partial^{+}\right)$be arbitrary, and we attempt to show that $\mathbf{P} f(F)(k) \leq \mathbf{P} f\left(F^{\prime}\right)(k)+\alpha r$. Since $k \circ f$ is in $\mathcal{L}_{\alpha}(X, d), 1 / \alpha(k \circ f)$ is in $\mathcal{L}_{1}(X, d)$. We expand the definition of $d_{\mathrm{KR}}$ in $d_{\mathrm{KR}}\left(F, F^{\prime}\right) \leq r$, and we obtain that $F(1 / \alpha(k \circ f)) \leq F^{\prime}(1 / \alpha(k \circ f))+r$. Using positive homogeneity, we deduce $F(k \circ f) \leq F^{\prime}(k \circ f)+\alpha r$, which is what we wanted to prove.

The proof that $\mathbf{P} f$ is $\alpha$-Lipschitz from $\mathbf{P} X, d_{\mathrm{KR}}^{a}$ to $\mathbf{P} Y, \partial_{\mathrm{KR}}^{a}$ is the same, noticing that if $k$ is bounded by $a$, then $k \circ f$ is also bounded by $a$.

The one thing that is missing in order to show that $\mathbf{P}$ is a functor, in a category whose morphisms are 1-Lipschitz continuous maps, is continuity. For now, we have the following approximation, which shows that naive suprema are preserved.

Lemma 4.22. Let $X, d$ and $Y, \partial$ be two quasi-metric spaces, and $f: X, d \mapsto$ $Y, \partial$ be a 1-Lipschitz continuous map. For every directed family of formal balls $\left(F_{i}, r_{i}\right), i \in I$, in $\mathbf{B}\left(\mathbf{P} X, d_{K R}\right)$ (resp., $\left.d_{K R}^{a}\right)$, with naive supremum $(F, r)$, $(\mathbf{P} f(F), r)$ is the naive supremum of $\left(\mathbf{P} f\left(F_{i}\right), r_{i}\right)_{i \in I}$ in $\mathbf{B}\left(\mathbf{P} Y, \partial_{K R}\right)$ (resp., $\left.\partial_{K R}^{a}\right)$.

Proof. By definition, $r=\inf _{i \in I} r_{i}$, and $F(h)=\sup _{i \in I}\left(F_{i}(h)+\alpha r-\alpha r_{i}\right)$ for every $h \in \mathcal{L}_{\alpha}(X, d)$ (resp., in $\mathcal{L}_{\alpha}^{a}(X, d)$ ), for every $\alpha \in \mathbb{R}_{+}, \alpha>0$. It follows that, for every $k \in \mathcal{L}_{\alpha}(Y, \partial)$ (resp., in $\mathcal{L}_{\alpha}^{a}(Y, \partial)$ ), and using the fact that $k \circ f$ is in $\mathcal{L}_{\alpha}(X, d)$ (resp., in $\mathcal{L}_{\alpha}^{a}(X, d)$, since $k \circ f$ is bounded by $\alpha a$, just like $k), \mathbf{P} f(F)(k)=F(k \circ f)=\sup _{i \in I}\left(F_{i}(k \circ f)+\alpha r-\alpha r_{i}\right)=$ $\sup _{i \in I}\left(\mathbf{P} f\left(F_{i}\right)(k)+\alpha r-\alpha r_{i}\right)$. 
If $X, d$ is standard, then $\eta_{X}$ is 1-Lipschitz continuous [10, Lemma 3.8]. In fact, it is the unit of the formal ball monad $\mathbf{B}$ on the category of standard quasi-metric spaces and 1-Lipschitz continuous maps [10, Theorem 3.14]. (Note that the latter implies that $\mathbf{B}$ preserves standardness, hence that $\mathbf{B}(X, d), d^{+}$is standard.) Hence Lemma 4.21 implies that $\mathbf{P} \eta_{X}(F)$ is a prevision on $\mathbf{B}(X, d), d^{+}$, which one can see as an extension of $F$ from $X$ to the superspace $\mathbf{B}(X, d)$. Moreover, $\mathbf{P} \eta_{X}$ is 1-Lipschitz, so $\mathbf{B}^{1}\left(\mathbf{P} \eta_{X}\right)$ is monotonic. This implies that for every directed family $\left(F_{i}, r_{i}\right)_{i \in I}$ in $\mathbf{B}\left(\mathbf{P} X, d_{\mathrm{KR}}\right)$, $\left(\mathbf{P} \eta_{X}\left(F_{i}\right), r_{i}\right)_{i \in I}$ is also a directed family, this time in $\mathbf{B}\left(\mathbf{P}(\mathbf{B}(X, d)), d^{+} \mathrm{KR}\right)$. Because $\mathbf{B}(X, d), d^{+}$is Lipschitz regular [10, Theorem 4.12], and standard, as we have noticed, $\left(\mathbf{P} \eta_{X}\left(F_{i}\right), r_{i}\right)_{i \in I}$ has a supremum $(G, r)$, as an application of Theorem 4.8. That supremum $(G, r)$ is the naive supremum, which is to say that $r=\inf _{i \in I} r_{i}$, and $G$ satisfies Equation (4), for every $k \in \mathcal{L}_{\alpha}\left(\mathbf{B}(X, d), d^{+}\right)$, $\alpha>0$ (resp., $\mathcal{L}_{\alpha}^{a}\left(\mathbf{B}(X, d), d^{+}\right)$, in the $d_{\mathrm{KR}}^{a}$ case).

Lemma 4.23. Let $X, d$ be a standard quasi-metric space, $\left(F_{i}, r_{i}\right)_{i \in I}$ be a directed family in $\mathbf{B}\left(\mathbf{P} X, d_{K R}\right)$ (resp., $\left.d_{K R}^{a}\right)$, let $r \stackrel{\text { def }}{=} \inf _{i \in I} r_{i}$ and $G$ be the prevision on $\mathbf{B}(X, d)$ defined as the naive supremum of $\left(\mathbf{P} \eta_{X}\left(F_{i}\right), r_{i}\right)_{i \in I}$. For every $\epsilon>0, G$ is supported on $V_{\epsilon}$.

Proof. Direct consequence of Lemma 4.20.

We make the stronger assumption that $G$ is supported on $X$ in the following proposition.

Proposition 4.24. Let $X, d$ be a standard quasi-metric space, $\left(F_{i}, r_{i}\right)_{i \in I}$ be a directed family in $\mathbf{B}\left(\mathbf{P} X, d_{K R}\right)$ (resp., $d_{K R}^{a}$ ), let $r \stackrel{\text { def }}{=} \inf _{i \in I} r_{i}$ and $G$ be the prevision on $\mathbf{B}(X, d)$ defined as the naive supremum of $\left(\mathbf{P} \eta_{X}\left(F_{i}\right), r_{i}\right)_{i \in I}$. If $G$ is supported on $X$, then:

1. $G=\mathbf{P} \eta_{X}(F)$ for some unique prevision $F$ on $X$;

2. if $G$ is superlinear, resp. sublinear, resp. linear, resp. subnormalized, resp. normalized, resp. discrete, then so is $F$;

3. $(F, r)$ is the naive supremum of $\left(F_{i}, r_{i}\right)_{i \in I}$ in $\mathbf{B}\left(\mathbf{P} X, d_{K R}\right)$ (resp., $\left.d_{K R}^{a}\right)$;

4. $(F, r)$ is the supremum of $\left(F_{i}, r_{i}\right)_{i \in I}$ in $\mathbf{B}\left(\mathbf{P} X, d_{K R}\right)$ (resp., $\left.d_{K R}^{a}\right)$. 
Proof. 1. This is Lemma 4.18, which also shows that $F(f)=G(\hat{f})$ for every $f \in \mathcal{L} X$.

2. One may observe that item 2 is a consequence of item 3, using Proposition 4.7 , item 4 , to show that naive suprema of directed families of formal balls of previsions on $\mathbf{B}(X, d)$ preserve superlinearity, sublinearity, etc. However, a direct proof is equally simple. Using the fact that $\widehat{g}+\widehat{h}$ and $\widehat{g+h}$ have the same restriction $g+h$ to $X$, we obtain that $F$ is superlinear, resp. sublinear, resp. linear, resp. subnormalized, resp. normalized if $G$ is. It remains to show that If $G$ is discrete, then $F$ is discrete as well. Let $f$ be any strict map in $\mathcal{L} \overline{\mathbb{R}}_{+}$. Then $F(f \circ h)=G(\widehat{f \circ h})$. We have $\widehat{f \circ h}(x, 0)=f(h(x))=f(\widehat{h}(x, 0))$, so $\widehat{f \circ h}$ and $f \circ \widehat{h}$ have the same restriction to $X$. It follows that $F(f \circ h)=G(f \circ \widehat{h})=f(G(\widehat{h}))=f(F(h))$, showing that $F$ is discrete.

3. For every $h \in \mathcal{L}_{\alpha}(X, d)$ (resp., $\mathcal{L}_{\alpha}^{a}(X, d)$ in the case of $d_{\mathrm{KR}}^{a}$ ), $\alpha>0$, $F(h)=G(\widehat{h})$ by definition. Let $k: \mathbf{B}(X, d) \rightarrow \overline{\mathbb{R}}_{+}$be defined by $k(x, r) \stackrel{\text { def }}{=}$ $\max (h(x)-\alpha r, 0)$. In other words, $k(x, r)=\max \left(h^{\prime}(x, r), 0\right) ; h^{\prime}:(x, r) \mapsto$ $h(x)-\alpha r$ was introduced in Section 2.2, and is Scott-continuous as soon as $h$ is $\alpha$-Lipschitz continuous [10, Lemma 2.7]. Hence $k$ is Scott-continuous.

It turns out that the $d^{+}$-Scott topology on $\mathbf{B}(X, d)$ coincides with the Scott topology of $\leq^{d^{+}}$, see [7, Exercise 7.4.53] or [10, Lemma 3.7]. Since the $\mathrm{d}_{\mathbb{R}^{-}}$Scott topology on $\overline{\mathbb{R}}_{+}$also coincides with the Scott topology, $k$ is continuous from $\mathbf{B}(X, d)$ with the $d^{+}$-Scott topology to $\overline{\mathbb{R}}_{+}$with the $\mathbf{d}_{\mathbb{R}}$ topology.

We claim that $k$ is $\alpha$-Lipschitz. Since $\mathbf{B}(X, d), d^{+}$is standard, as we have recalled before Lemma 4.23 , and since $\overline{\mathbb{R}}_{+}, d_{\mathbb{R}}$ is standard (since complete), and $k$ is continuous, this will imply that $k$ is $\alpha$-Lipschitz continuous. For every $a \in \mathbb{R}_{+}$, if $d^{+}((x, r),(y, s)) \leq a$, namely if $d(x, y) \leq r-s+a$, then $h(x)-\alpha r \leq h(y)+\alpha(r-s+a)-\alpha r=h(y)-\alpha s+\alpha a$, so $k(x, r) \leq$ $\max (h(y)-\alpha s+\alpha a, 0) \leq \max (h(y)-\alpha s+\alpha a, \alpha a)=k(y, s)+\alpha a$, showing that $k$ is $\alpha$-Lipschitz.

Taking $r \stackrel{\text { def }}{=} 0$ in the definition of $k$, we see that $k(x, 0)=h(x)$, hence that $k \circ \eta_{X}=h$.

Since $k$ is $\alpha$-Lipschitz continuous, we can use (4), to the effect that $G(k)=$ $\sup _{i \in I}\left(F_{i}\left(k \circ \eta_{X}\right)+\alpha r-\alpha r_{i}\right)=\sup _{i \in I}\left(F_{i}(h)+\alpha r-\alpha r_{i}\right)$. (In the case of $d_{\mathrm{KR}}^{a}$, this requires the verification that $k$ is bounded from above by $\alpha a$, which is obvious, since that is already the case for $h$.) Now $G=\mathbf{P} \eta_{X}(F)$ by item 1, so $G(k)=F\left(k \circ \eta_{X}\right)=F(h)$, whence $F(h)=\sup _{i \in I}\left(F_{i}(h)+\alpha r-\alpha r_{i}\right)$. That shows that $F$ is the naive supremum of $\left(F_{i}\right)_{i \in I}$. 
4. By Proposition 4.7, item 3 (and the isomorphism between $\mathcal{L} \mathbf{P} X$ and $\mathbf{P} X$ that ensues from Proposition 4.5, and the similar isomorphism that ensures from Lemma 4.12 in the case of $d_{\mathrm{KR}}^{a}$ ), any naive supremum of a directed family that happens to be continuous, such as $F$, is the supremum.

We recap what we know as follows. As we said, the remaining, nagging assumption on supports will have to be taken care of in each special case.

Proposition 4.25 (Conditional completeness). Let $X, d$ be a standard quasi-metric space. Let $S$ be any subset of the following properties on previsions: sublinearity, superlinearity, linearity, subnormalization, normalization, discreteness; let $Y$ denote the set of previsions on $X$ satisfying $S$, and $Z$ be the set of previsions on $\mathbf{B}(X, d)$ that satisfy $S$.

If every element of $Z$ that is supported on $V_{1 / 2^{n}}$ for every $n \in \mathbb{N}$ is in fact supported on $X$, then $Y, d_{K R}$ is complete, and directed suprema in $\mathbf{B}\left(Y, d_{K R}\right)$ are computed as naive suprema.

The same result holds for $d_{K R}^{a}$ in lieu of $d_{K R}$, for any $a>0$.

\section{The Hoare Hyperspace}

\subsection{The $d_{\mathcal{H}}$ Quasi-Metric}

Through the bijection $C \mapsto F^{C}$ of Proposition 3.2, it makes sense to define a quasi-metric $d_{\mathcal{H}}$ on $\mathcal{H}_{0} X$, and on $\mathcal{H} X$, by $d_{\mathcal{H}}\left(C, C^{\prime}\right)=d_{\mathrm{KR}}\left(F^{C}, F^{C^{\prime}}\right)$. We will show that (provided $X, d$ is standard), it is equivalent to define $d_{\mathcal{H}}$ as in Definition 5.3 below, which should be reminiscent of one half of the Hausdorff metric.

For every open subset $U$ of $X$ (with its $d$-Scott topology), and every point $x \in X$, we define the distance from $x$ to the complement $C \stackrel{\text { def }}{=} \bar{U}$ of $U$ by $d(x, C) \stackrel{\text { def }}{=} \sup \left\{r \in \mathbb{R}_{+} \mid(x, r) \in \widehat{U}\right\}[13$, Definition 6.10]. (The supremum of the empty set is 0 , the supremum of an unbounded set is $+\infty$.) We recall that $\widehat{U}$ is the largest open subset of $\mathbf{B}(X, d)$ such that $\widehat{U} \cap X=U$.

The following laws hold [13, Lemma 6.11]:

Fact 5.1. If $X, d$ is standard, then for all $x, y \in X$ and for every closed subset $C$ of $X: d(x, C)=0$ if and only if $x \in C ; d(x, C) \leq d(x, y)+d(y, C)$; the map $d\left({ }_{-}, C\right)$ is 1-Lipschitz continuous from $X, d$ to $\overline{\mathbb{R}}_{+}, \mathrm{d}_{\mathbb{R}}$. 
Additionally, $d(x, C) \leq \inf _{y \in C} d(x, y)$, with equality if $C=\downarrow E$ for some finite set $E$, or if $x$ is a center point [13, Proposition 6.12]. The latter will be important, and we state it as a fact.

Fact 5.2. If $X, d$ is standard, then for every center point $x$ of $X, d$, for every closed subset $C$ of $X$ in its d-Scott topology, $d(x, C)=\inf _{y \in C} d(x, y)$.

Since every point in a metric space (or in a Smyth-complete quasi-metric space) is a center point, we retrieve the familiar definition $d(x, C)=\inf _{y \in C} d(x, y)$ of the distance of a point to a closed set, when $d$ is a metric or a Smythcomplete quasi-metric; but beware that this definition is not the one that we need in more general quasi-metric situations, except when $x$ is a center point.

An equivalent definition, working directly with closed sets, is as follows. For a closed subset $C$ of $X$, let $c l_{\mathbf{B}}(C)$ be its closure in $\mathbf{B}(X, d)$ - the smallest closed subset of $\mathbf{B}(X, d)$ that contains $C$, seeing $X$ as a subspace of $\mathbf{B}(X, d)$. If $U$ is the complement of $C$, then $c l_{\mathbf{B}}(C)$ is the complement of $\widehat{U}$ in $\mathbf{B}(X, d)$. Then $d(x, C)=\sup \left\{r \in \mathbb{R}_{+} \mid(x, r) \notin c l_{\mathbf{B}}(C)\right\}$, and $d(x, C)=0$ if and only if $x \in C$ (Fact 5.1).

Definition $5.3\left(d_{\mathcal{H}}\right)$. Let $X, d$ be a standard quasi-metric space. For any two closed subsets $C, C^{\prime}$ of $X$, let $d_{\mathcal{H}}\left(C, C^{\prime}\right) \stackrel{\text { def }}{=} \sup _{x \in C} d\left(x, C^{\prime}\right)$.

Lemma 5.4. Let $X, d$ be a quasi-metric space. For every $x \in X$ and every closed subset $C$ of $X, d(x, C)=\inf \left\{r \in \mathbb{R}_{+} \mid(x, r) \in c l_{\mathbf{B}}(C)\right\}$, where the infimum of an empty set of elements of $\mathbb{R}_{+}$is taken to be $+\infty$.

Moreover,

1. the infimum is attained if $d(x, C)<+\infty$;

2. for every $r \in \mathbb{R}_{+}, d(x, C) \leq r$ if and only if $(x, r) \in c l_{\mathbf{B}}(C)$.

Proof. Let $A \stackrel{\text { def }}{=}\left\{r \in \mathbb{R}_{+} \mid(x, r) \notin c l_{\mathbf{B}}(C)\right\}, B=\left\{r \in \mathbb{R}_{+} \mid(x, r) \in\right.$ $\left.c l_{\mathbf{B}}(C)\right\}$. $A$ and $B$ partition $\mathbb{R}_{+}$, and since $c l_{\mathbf{B}}(C)$ is downwards-closed, $A$ is downwards-closed, $B$ is upwards-closed, and for all $a \in A, b \in B$, we must have $a<b$. It follows that $\sup A=\inf B$, proving the first claim.

$B$ is also closed under (filtered) infima. Indeed, for any filtered family $\left(r_{i}\right)_{i \in I}$ of elements of $B$ with $\inf _{i \in I} r_{i}=r,\left(x, r_{i}\right)_{i \in I}$ is a directed family in $\mathbf{B}(X, d)$, whose supremum is $(x, r)$. (The upper bounds of $\left(x, r_{i}\right)_{i \in I}$ are those 
formal balls $(y, s)$ such that $d(x, y) \leq r_{i}-s$ for every $i \in I$, namely the elements above $(x, r)$.) That implies that $r$ is in $B$.

It follows that, if $B$ is non-empty, then it is an interval of the form $[b,+\infty[$, where $b \stackrel{\text { def }}{=} \inf B=d(x, C)$. This shows item 1 .

For item 2 , if $d(x, C) \leq r \in \mathbb{R}_{+}$, then $B$ is non-empty and $r \geq b$, so $r$ is in $B$, that is, $(x, r) \in c l_{\mathbf{B}}(C)$. Conversely, if $(x, r) \in c l_{\mathbf{B}}(C)$, then $r$ is in $B$, so $B$ is non-empty and $r \geq b$, meaning that $d(x, C) \leq r$.

In the following, we will need to know a few elementary facts about the closure operation $c l_{\mathbf{B}}$. For a subset $\mathcal{A}$ of $\mathbf{B}(X, d)$, and every $a \in \mathbb{R}_{+}$, we agree that $\mathcal{A}+a$ denotes $\{(x, r+a) \mid(x, r) \in \mathcal{A}\}$.

Lemma 5.5. Let $X, d$ be a standard quasi-metric space.

1. For every Scott-closed subset $\mathcal{C}$ of $\mathbf{B}(X, d), \mathcal{C}+a$ is Scott-closed.

2. For every subset $\mathcal{A}$ of $\mathbf{B}(X, d), c l_{\mathbf{B}}(\mathcal{A})+a=c l_{\mathbf{B}}(\mathcal{A}+a)$.

ProOf. 1. For every formal ball $(x, r)$ below some element $(y, s+a)$ of $\mathcal{C}+a$ (viz., $(y, s)$ is in $\mathcal{C}), d(x, y) \leq r-s-a$, so $r \geq s+a \geq a$, and $(x, r-a) \leq^{d^{+}}(y, s)$. Since $\mathcal{C}$ is downwards-closed, $(x, r-a)$ is in $\mathcal{C}$, hence $(x, r)$ is in $\mathcal{C}+a$. This proves that $\mathcal{C}+a$ is downwards-closed. For every directed family $\left(x_{i}, r_{i}+a\right)_{i \in I}$ in $\mathcal{C}+a,\left(x_{i}, r_{i}\right)_{i \in I}$ is also directed, and in $\mathcal{C}$. If $\left(x_{i}, r_{i}+a\right)_{i \in I}$ has a supremum, then $\left(x_{i}, r_{i}\right)_{i \in I}$ also has a supremum $(x, r)$, by the definition of standardness, and the supremum of $\left(x_{i}, r_{i}+a\right)_{i \in I}$ is $(x, r+a)$. Since $\mathcal{C}$ is Scott-closed, $(x, r)$ is in $\mathcal{C}$, so $(x, r+a)$ is in $\mathcal{C}+a$.

2. Since $\operatorname{cl}_{\mathbf{B}}(\mathcal{A})+a$ is Scott-closed, and since it obviously contains $\mathcal{A}+a$, it contains $c l_{\mathbf{B}}(\mathcal{A}+a)$. For the reverse inclusion, we use the fact that the map - $+a$ is Scott-continuous, since $X, d$ is standard, and that for a continuous map, the closure of the image of a set contains the image of the closure.

In accordance with our convention that $X$ is equated with a subspace of $\mathbf{B}(X, d)$ through the embedding $\eta_{X}: x \mapsto(x, 0)$, we also agree that, for any subset $C$ of $X, C+a$ denotes $\{(x, a) \mid x \in C\}$.

Lemma 5.6. Let $X, d$ be a standard quasi-metric space. For every closed subset $C$ of $X$, for every $a \in \mathbb{R}_{+}$:

1. $\operatorname{cl}_{\mathbf{B}}(C) \cap X=C$;

2. $c l_{\mathbf{B}}(C)+a=c l_{\mathbf{B}}(C+a)$. 
Proof. 1. Let $U$ be the complement of $C$ in $X$, so that $c l_{\mathbf{B}}(C)$ is the complement of $\widehat{U}$ in $\mathbf{B}(X, d)$. Since $\widehat{U} \cap X=U$, by taking complements $c l_{\mathbf{B}}(C) \cap X=C$.

2. By Lemma 5.5, item 2.

Those preliminary lemmas allow us to give the following, practical tools to work with $d_{\mathcal{H}}$.

Lemma 5.7. Let $X, d$ be a standard quasi-metric space.

1. For every $C \in \mathcal{H}_{0} X$, for every closed set $C^{\prime}$, for every $a \in \mathbb{R}_{+}$, $d_{\mathcal{H}}\left(C, C^{\prime}\right) \leq a$ if and only if $C+a \subseteq c_{\mathbf{B}}\left(C^{\prime}\right)$;

2. $d_{\mathcal{H}}$ is a quasi-metric on $\mathcal{H}_{0} X$, and its specialization ordering $\leq^{d_{\mathcal{H}}}$ is inclusion;

3. the ordering $\leq^{d_{\mathcal{H}}^{+}}$on $\mathbf{B}\left(\mathcal{H}_{0} X, d_{\mathcal{H}}\right)$ is given by $(C, r) \leq d_{\mathcal{H}}^{+}\left(C^{\prime}, r^{\prime}\right)$ if and only if $C+r \subseteq c l_{\mathbf{B}}\left(C^{\prime}+r^{\prime}\right)$.

Proof. 1. By definition, $d_{\mathcal{H}}\left(C, C^{\prime}\right) \leq a$ if and only if, for every $x \in C$, $d\left(x, C^{\prime}\right) \leq a$, if and only if, for every $x \in C,(x, a)$ is in $c l_{\mathbf{B}}\left(C^{\prime}\right)$, by Lemma 5.4, item 2. That is equivalent to $C+a \subseteq c l_{\mathbf{B}}\left(C^{\prime}\right)$.

2. By item $1, d_{\mathcal{H}}\left(C, C^{\prime}\right)=0$ if and only if $C \subseteq \operatorname{cl}_{\mathbf{B}}\left(C^{\prime}\right)$. The latter is equivalent to $C \subseteq c l_{\mathbf{B}}\left(C^{\prime}\right) \cap X$, hence to $C \subseteq C^{\prime}$, using Lemma 5.6, item 1 . This shows immediately that $d_{\mathcal{H}}\left(C, C^{\prime}\right)=\bar{d}_{\mathcal{H}}\left(C^{\prime}, C\right)=0$ implies $C=C^{\prime}$, and that inclusion will be the specialization ordering $\leq{ }^{d_{\mathcal{H}}}$ of $d_{\mathcal{H}}$.

It still remains to show the triangular inequality, namely $d_{\mathcal{H}}\left(C, C^{\prime \prime}\right) \leq$ $d_{\mathcal{H}}\left(C, C^{\prime}\right)+d_{\mathcal{H}}\left(C^{\prime}, C^{\prime \prime}\right)$. If the right-hand side is equal to $+\infty$, then the inequality is obvious. Otherwise, let $a \stackrel{\text { def }}{=} d_{\mathcal{H}}\left(C, C^{\prime}\right), a^{\prime}=d_{\mathcal{H}}\left(C^{\prime}, C^{\prime \prime}\right)$, both elements of $\mathbb{R}_{+}$. By item $1, C+a \subseteq c l_{\mathbf{B}}\left(C^{\prime}\right)$ and $C^{\prime}+a^{\prime} \subseteq c l_{\mathbf{B}}\left(C^{\prime \prime}\right)$. Therefore $C+a+a^{\prime} \subseteq c l_{\mathbf{B}}\left(C^{\prime}\right)+a^{\prime}=c l_{\mathbf{B}}\left(C^{\prime}+a^{\prime}\right)$ (by Lemma 5.6, item 2) $\subseteq c l_{\mathbf{B}}\left(C^{\prime \prime}\right)$. By item 1 again, this implies that $d_{\mathcal{H}}\left(C, C^{\prime \prime}\right) \leq a+a^{\prime}$, which proves our claim.

3. $(C, r) \leq^{d_{\mathcal{H}}^{+}}\left(C^{\prime}, r^{\prime}\right)$ if and only if $r \geq r^{\prime}$ and $C+r-r^{\prime} \subseteq c l_{\mathbf{B}}\left(C^{\prime}\right)$, by item 1 . Adding $r^{\prime}$ to both sides, $C+r-r^{\prime} \subseteq c l_{\mathbf{B}}\left(C^{\prime}\right)$ if and only if $C+r \subseteq c l_{\mathbf{B}}\left(C^{\prime}\right)+r^{\prime}$, and $c l_{\mathbf{B}}\left(C^{\prime}\right)+r^{\prime}=c l_{\mathbf{B}}\left(C^{\prime}+r^{\prime}\right)$ by Lemma 5.6, item 2 .

We can now show that $d_{\mathcal{H}}$ and $d_{\mathrm{KR}}$ are related as expected.

Proposition 5.8. Let $X, d$ be a standard quasi-metric space. The bijection $C \mapsto F^{C}$ is an isometry between $\mathcal{H}_{0} X$ and the set of discrete sublinear previsions on $X$ : for all closed subsets $C, C^{\prime}, d_{\mathcal{H}}\left(C, C^{\prime}\right)=d_{K R}\left(F^{C}, F^{C^{\prime}}\right)$. 
ProOF. This is a bijection by Proposition 3.2.

We take $h \stackrel{\text { def }}{=} d\left({ }_{-}, C^{\prime}\right)$ in the definition of $d_{\mathrm{KR}}$ (Equation $\left.(1)\right)$, and we recall that this is 1-Lipschitz continuous. We obtain that $d_{\mathrm{KR}}\left(F^{C}, F^{C^{\prime}}\right) \geq$ $\mathrm{d}_{\mathbb{R}}\left(\sup _{x \in C} d\left(x, C^{\prime}\right), \sup _{y \in C^{\prime}} d\left(y, C^{\prime}\right)\right)=\mathrm{d}_{\mathbb{R}}\left(d_{\mathcal{H}}\left(C, C^{\prime}\right), d_{\mathcal{H}}\left(C^{\prime}, C^{\prime}\right)\right)=\mathrm{d}_{\mathbb{R}}\left(d_{\mathcal{H}}\left(C, C^{\prime}\right), 0\right)$ (using Lemma 5.7, item 2) $=d_{\mathcal{H}}\left(C, C^{\prime}\right)$.

For the reverse inclusion, fix $h \in \mathcal{L}_{1}(X, d)$, and let us check that $\sup _{x \in C} h(x) \leq$ $\sup _{y \in C^{\prime}} h(y)+d_{\mathcal{H}}\left(C, C^{\prime}\right)$. If $d_{\mathcal{H}}\left(C, C^{\prime}\right)=+\infty$, this is clear, so we assume $a \stackrel{\text { def }}{=} d_{\mathcal{H}}\left(C, C^{\prime}\right)<+\infty$. We fix $x \in C$. Our aim is to show that $h(x)-a \leq$ $\sup _{y \in C^{\prime}} h(y)$. Note that the left-hand side is $h^{\prime}(x, a)$, where $h^{\prime}:(z, t) \mapsto$ $h(z)-t$ is Scott-continuous. For every $b<h^{\prime}(x, a)$, the formal ball $(x, a)$ is in the Scott-open set $\left.\left.h^{\prime-1}(] b,+\infty\right]\right)$. Using Lemma 5.7, item 1, and the fact that $a=d_{\mathcal{H}}\left(C, C^{\prime}\right), C+a$ is included in $c l_{\mathbf{B}}\left(C^{\prime}\right)$, so $(x, a)$ is in $c l_{\mathbf{B}}\left(C^{\prime}\right)$. Therefore $\left.\left.h^{\prime-1}(] b,+\infty\right]\right)$ intersects $c l_{\mathbf{B}}\left(C^{\prime}\right)$, hence also $C^{\prime}$. Let $y$ be in the intersection. Then $h^{\prime}(y, 0)=h(y)>b$, which shows that $\sup _{y \in C^{\prime}} h(y)>b$. As $b<h^{\prime}(x, a)$ is arbitrary, we conclude.

Remark 5.9. By using similar arguments, one can show that $d_{K R}^{a}\left(F^{C}, F^{C^{\prime}}\right)=$ $d_{\mathcal{H}}^{a}\left(C, C^{\prime}\right)$, where $d_{\mathcal{H}}^{a}\left(C, C^{\prime}\right)$ is defined as $\min \left(a, d_{\mathcal{H}}\left(C, C^{\prime}\right)\right)$, for every a $\in \mathbb{R}_{+}$, $a>0$. In one direction, $d_{K R}^{a}\left(F^{C}, F^{C^{\prime}}\right) \leq d_{K R}\left(F^{C}, F^{C^{\prime}}\right)=d_{\mathcal{H}}\left(C, C^{\prime}\right)$ by Proposition 5.8, and $d_{K R}^{a}\left(F^{C}, F^{C^{\prime}}\right) \leq a$ by definition. In the other direction, we take $h=\min \left(a, d\left({ }_{-}, C^{\prime}\right)\right)$ in the definition of $d_{K R}^{a}$ (3), check that $h \in$ $\mathcal{L}_{1}^{a}(X, d)$, and therefore obtain that $d_{K R}^{a}\left(F^{C}, F^{C^{\prime}}\right)$ is larger than or equal to $\mathrm{d}_{\mathbb{R}}\left(\sup _{x \in C} \min \left(a, d\left(x, C^{\prime}\right)\right), \sup _{y \in C^{\prime}} \min \left(a, d\left(y, C^{\prime}\right)\right)\right)=\min \left(a, d\left(C, C^{\prime}\right)\right)$, using Fact 5.1.

\subsection{Completeness}

We wish to apply Proposition 4.25. To this end, we verify its funny condition on supports. We require the following folklore lemma.

Lemma 5.10. For every topological space $Y$, for every subset $A$ of $Y$, for every lower semicontinuous map $h: Y \rightarrow \mathbb{R} \cup\{-\infty,+\infty\} \sup _{y \in A} h(y)=$ $\sup _{y \in c l(A)} h(y)$.

Proof. Since $A \subseteq c l(A), \sup _{y \in A} h(y) \leq \sup _{y \in c l(A)} h(y)$. If that inequality were strict, then there would be a real number $a$ such that $\sup _{y \in A} h(y) \leq a<$ $\sup _{y \in c l(A)} h(y)$. In particular, $\left.\left.h^{-1}(] a,+\infty\right]\right)$ would intersect $c l(A)$, hence also $A$. Therefore, there would be an $y \in A$ such that $h(y)>a$, which contradicts $\sup _{y \in A} h(y) \leq a$. 
Lemma 5.11. Let $X, d$ be a continuous complete quasi-metric space. For every closed subset $\mathfrak{C}$ of $\mathbf{B}(X, d)$ such that $F^{\mathfrak{C}}$ is supported on $V_{1 / 2^{n}}$ for every $n \in \mathbb{N}$,

1. $\mathfrak{C}$ is equal to $\mathrm{cl}_{\mathbf{B}}(C)$, where $C$ is the closed set $\mathfrak{C} \cap X$;

2. For every $k \in \mathcal{L}(\mathbf{B}(X, d)), F^{\mathfrak{C}}(k)=F^{C}\left(k \circ \eta_{X}\right)$;

3. $F^{\mathfrak{C}}$ is supported on $X$.

Proof. We first note that for every (Scott-)open subset $\mathcal{U}$ of $\mathbf{B}(X, d)$ that intersects $\mathfrak{C}$, for every $\eta>0, \mathfrak{C}$ also intersects $\mathcal{U} \cap V_{\eta}$. Indeed, since $\mathcal{U} \cap \mathfrak{C} \neq \emptyset$, $F^{\mathfrak{C}}\left(\chi_{\mathcal{U}}\right)=1$. We use our assumption that $F^{\mathfrak{C}}$ is supported on $V_{1 / 2^{n}}$, where we choose $n$ so large that $1 / 2^{n}<\eta$ : since $\chi_{\mathcal{U}}$ and $\chi_{\mathcal{U} \cap V_{\eta}}$ coincide on $V_{1 / 2^{n}}$, $F^{\mathfrak{C}}\left(\chi_{\mathcal{U} \cap V_{\eta}}\right)=1$. Therefore $\mathfrak{C}$ also intersects $\mathcal{U} \cap V_{\eta}$.

1. Clearly $c l_{\mathbf{B}}(C) \subseteq \mathfrak{C}$. In the reverse direction, we assume for the sake of contradiction that $\mathfrak{C}$ is not included in $\operatorname{cl}_{\mathbf{B}}(C)$. There is a formal ball $(x, r)$ in $\mathfrak{C}$ that is in some Scott-open subset $\mathcal{U}$ (namely, the complement of $c l_{\mathbf{B}}(C)$ ) such that $\mathcal{U} \cap X$ does not intersect $C$.

By our preliminary remark, $\mathfrak{C}$ must also intersect $\mathcal{U} \cap V_{1}$, say at $\left(y_{0}, s_{0}\right)$. Since $\mathbf{B}(X, d)$ is a continuous dcpo, $\left(y_{0}, s_{0}\right)$ is the supremum of a directed family of formal balls way-below $\left(y_{0}, s_{0}\right)$, so one of them, call it $\left(x_{0}, r_{0}\right)$, is in $\mathcal{U} \cap V_{1}$. It is also in $\mathfrak{C}$, since every Scott-closed set is downwards-closed.

Since $\left(x_{0}, r_{0}\right) \ll\left(y_{0}, s_{0}\right), \mathfrak{C} \cap \uparrow\left(x_{0}, r_{0}\right)$ is non-empty. By our preliminary remark again, $\mathfrak{C}$ must intersect $\uparrow\left(x_{0}, r_{0}\right) \cap V_{1 / 2}$, say at $\left(y_{1}, s_{1}\right)$. As above, there is formal ball $\left(x_{1}, r_{1}\right) \ll\left(y_{1}, s_{1}\right)$ in $\mathfrak{C} \cap \uparrow\left(x_{0}, r_{0}\right) \cap V_{1 / 2}$.

We proceed in the same way: $\mathfrak{C} \cap \uparrow\left(x_{1}, r_{1}\right)$ is non-empty, hence $\mathfrak{C} \cap$ $\uparrow\left(x_{1}, r_{1}\right) \cap V_{1 / 4}$ contains a formal ball $\left(y_{2}, s_{2}\right)$, and that allows us to find $\left(x_{2}, r_{2}\right) \ll\left(y_{2}, s_{2}\right)$ in $\mathfrak{C} \cap \uparrow\left(x_{1}, r_{1}\right) \cap V_{1 / 4}$.

Inductively, we obtain formal balls $\left(x_{n}, r_{n}\right)$ in $\mathfrak{C} \cap V_{1 / 2^{n}}$ with the property that $\left(x_{0}, r_{0}\right) \ll\left(x_{1}, r_{1}\right) \ll \cdots \ll\left(x_{n}, r_{n}\right) \ll \cdots$, and $\left(x_{0}, r_{0}\right) \in \mathcal{U}$. Let $(y, s) \stackrel{\text { def }}{=} \sup _{n \in \mathbb{N}}\left(x_{n}, r_{n}\right)$. Since $s \leq r_{n}$ and $\left(x_{n}, r_{n}\right) \in V_{1 / 2^{n}}, s$ is less than or equal to $1 / 2^{n}$ for every $n \in \mathbb{N}$, hence zero. Since $\mathfrak{C}$ is Scott-closed, $(y, s)=$ $(y, 0)$ is in $\mathfrak{C}$, so $y$ is in $C$. Because $\mathcal{U}$ is upwards-closed and $\left(x_{0}, r_{0}\right)$ is in $\mathcal{U}$, $(y, 0)$ must also be in $\mathcal{U}$. But that contradicts the fact that $\mathcal{U} \cap X$ does not intersect $C$.

2. Let $k \in \mathcal{L}(\mathbf{B}(X, d))$. We have $F^{\mathfrak{C}}(k)=\sup _{(x, r) \in \mathfrak{C}} k(x, r)=\sup _{(x, r) \in c l_{\mathbf{B}}(C)} k(x, r)$ by item 1 , so $F^{\mathfrak{C}}(k)=\sup _{x \in C} k(x, 0)$ by Lemma 5.10 , and that is exactly $F^{C}\left(k \circ \eta_{X}\right)$. 
3. Let $g, f \in \mathcal{L}(\mathbf{B}(X, d))$ be such that $g_{\mid X}=f_{\mid X}$, that is, $g \circ \eta_{X}=f \circ \eta_{X}$. By item $2, F^{\mathfrak{C}}(g)=F^{C}\left(g \circ \eta_{X}\right)=F^{C}\left(f \circ \eta_{X}\right)=F^{\mathfrak{C}}(f)$.

Proposition 5.12 (Completeness, Hoare hyperspace). Let X, d be a standard Lipschitz regular quasi-metric space, or a continuous complete quasimetric space. Then $\mathcal{H}_{0} X, d_{\mathcal{H}}$ and $\mathcal{H} X, d_{\mathcal{H}}$ are complete.

Suprema of directed families of formal balls of previsions are naive suprema.

Proof. Through the isometry of Proposition 5.8, we only have to show that the spaces of discrete sublinear previsions (resp., the normalized discrete sublinear previsions) are complete under $d_{\mathrm{KR}}$. If $X, d$ is standard and Lipschitz regular, this follows from Theorem 4.8. Otherwise, this follows from Proposition 4.25 , whose assumption on supports is verified by Lemma 5.11.

Remark 5.13. Under the same assumptions, for every $a>0, \mathcal{H}_{0} X, d_{\mathcal{H}}^{a}$ and $\mathcal{H} X, d_{\mathcal{H}}^{a}$ are complete, and directed suprema of formal balls are naive suprema. The quasi-metric $d_{\mathcal{H}}^{a}$ was introduced in Remark 5.9. The argument is similar to Proposition 5.12, replacing Proposition 5.8 by Remark 5.9.

Remark 5.14. For $a>0$, it is clear that the specialization ordering $\leq_{\mathcal{H}}^{a}$ is again inclusion: $d_{\mathcal{H}}^{a}\left(C, C^{\prime}\right)=0$ if and only if $\min \left(a, d_{\mathcal{H}}\left(C, C^{\prime}\right)\right)=0$ if and only if $d_{\mathcal{H}}\left(C, C^{\prime}\right)=0$, if and only if $C \subseteq C^{\prime}$ by Lemma 5.7, item 2.

\section{3. $d_{\mathcal{H}}$-Limits}

Suprema of directed families of formal balls in $\mathcal{H}_{0} X$ and in $\mathcal{H} X$ are naive suprema, but the detour through previsions makes that less than explicit. Let us give a more concrete description of those suprema, i.e., of $d_{\mathcal{H}}$-limits.

Lemma 5.15. Let $X, d$ be a continuous complete quasi-metric space. For every directed family $\left(C_{i}, r_{i}\right)_{i \in I}$ in $\mathbf{B}\left(\mathcal{H}_{0}(X, d), d_{\mathcal{H}}\right)$, its supremum $(C, r)$ is given by $r \stackrel{\text { def }}{=} \inf _{i \in I} r_{i}$ and $C \stackrel{\text { def }}{=} c l_{\mathbf{B}}\left(\bigcup_{i \in I} C_{i}+r_{i}-r\right) \cap X$.

Proof. By Proposition 5.12, that supremum is the naive supremum, so $r=\inf _{i \in I} r_{i}$ in particular. One might think of writing down the naive supremum explicitly and simplifying the expression, but a direct verification seems easier.

Let $\mathcal{A} \stackrel{\text { def }}{=} \bigcup_{i \in I} C_{i}+r_{i}-r$ and $\mathfrak{C} \stackrel{\text { def }}{=} c l_{\mathbf{B}}(\mathcal{A})$. Let also $i \sqsubseteq j$ if and only if $\left(C_{i}, r_{i}\right) \leq d_{\mathcal{H}}^{+}\left(C_{j}, r_{j}\right)$, making $\left(C_{i}, r_{i}\right)_{i \in I, \subseteq}$ a monotone net. The core of the 
proof consists in showing that $\mathfrak{C}=c l_{\mathbf{B}}(C)$ for $C \stackrel{\text { def }}{=} \mathfrak{C} \cap X$, and we shall obtain that by showing that $F^{\mathfrak{C}}$ is supported on every $V_{\epsilon}, \epsilon>0$.

For every $k \in \mathcal{L}(\mathbf{B}(X, d)), F^{\mathfrak{C}}(k)=\sup _{(x, s) \in \mathcal{A}} k(x, s)$ by Lemma 5.10. By definition of $\mathcal{A}$, and another recourse to Lemma 5.10, $F^{\mathfrak{C}}(k)=\sup _{i \in I} \sup _{x \in C_{i}} k\left(x, r_{i}-\right.$ $r)=\sup _{i \in I} \sup _{(x, s) \in c l_{\mathbf{B}}\left(C_{i}+r_{i}-r\right)} k(x, s)$. If $i \sqsubseteq j$ then $d_{\mathcal{H}}\left(C_{i}, C_{j}\right) \leq r_{i}-r_{j}=$ $\left(r_{i}-r\right)-\left(r_{j}-r\right)$, whence $\left(C_{i}, r_{i}-r\right) \leq d_{\mathcal{H}}^{+}\left(C_{j}, r_{j}-r\right)$ and therefore $C_{i}+r_{i}-r \subseteq$ $c l_{\mathbf{B}}\left(C_{j}+r_{j}-r\right)$ by Lemma 5.7, item 3. In particular, $\sup _{x \in C_{i}} k\left(x, r_{i}-r\right)$ is smaller than or equal to $\sup _{x \in C_{j}} k\left(x, r_{j}-r\right)=\sup _{(x, s) \in c l_{\mathbf{B}}\left(C_{j}+r_{j}-r\right)} k(x, s)$. This shows that the outer supremum in $F^{\mathfrak{C}}(k)=\sup _{i \in I} \sup _{x \in C_{i}} k\left(x, r_{i}-r\right)$ is a directed supremum.

For any $i_{0} \in I$, the subfamily of all $i \in I$ such that $i_{0} \sqsubseteq i$ is cofinal in $I$, hence $F^{\mathfrak{C}}(k)$ is also equal to $\sup _{i \in I, i_{0} \sqsubseteq i} \sup _{x \in C_{i}} k\left(x, r_{i}-r\right)$.

For every $\epsilon>0$, if $g$ and $f$ are two elements of $\mathcal{L}(\mathbf{B}(X, d))$ that coincide on $V_{\epsilon}$, and since $r=\inf _{i \in r_{i}}$, there is an $i_{0} \in I$ such that $r_{i}-r<\epsilon$ for every $i \in I, i_{0} \sqsubseteq i$. Then $g\left(x, r_{i}-r\right)=f\left(x, r_{i}-r\right)$, and the formula we have just obtained for $F^{\mathfrak{C}}(k)$ shows that $F^{\mathfrak{C}}(g)=F^{\mathfrak{C}}(f)$. In other words, $F^{\mathfrak{C}}$ is supported on $V_{\epsilon}$. By Lemma $5.11, \mathfrak{C}$ is equal to $c l_{\mathbf{B}}(C)$, where $C \stackrel{\text { def }}{=} \mathfrak{C} \cap X$.

For every $i \in I, C_{i}+r_{i} \subseteq c l_{\mathbf{B}}(C+r)$, since $c l_{\mathbf{B}}(C+r)=c l_{\mathbf{B}}(C)+r$ (by Lemma 5.6, item 2) $=\mathfrak{C}+r=c_{\mathbf{B}}\left(\bigcup_{i \in I} C_{i}+r_{i}\right)$ (by Lemma 5.5, item 2, and the definition of $\mathfrak{C})$. Hence $(C, r)$ is an upper bound of $\left(C_{i}, r_{i}\right)_{i \in I}$, using Lemma 5.7, item 3.

Let us consider any other upper bound $\left(C^{\prime}, r^{\prime}\right)$ of $\left(C_{i}, r_{i}\right)_{i \in I}$. Then $r^{\prime} \leq$ $\inf _{i \in I} r_{i}=r$, and $C_{i}+r_{i} \subseteq c l_{\mathbf{B}}\left(C^{\prime}+r^{\prime}\right)$ for every $i \in I$, by Lemma 5.7, item 3 . Let $\mathfrak{D}$ be the set of formal balls $(y, s)$ such that $(y, s+r) \in c l_{\mathbf{B}}\left(C^{\prime}+r^{\prime}\right)$. Since $X, d$ is standard, the map $(y, s) \mapsto(y, s+r)$ is Scott-continuous, so $\mathfrak{D}$ is closed in $\mathbf{B}(X, d)$. Since $r_{i} \geq r$, the inclusion $C_{i}+r_{i} \subseteq c l_{\mathbf{B}}\left(C^{\prime}+r^{\prime}\right)$ rewrites as $C_{i}+r_{i}-r \subseteq \mathfrak{D}$. Taking unions over $i \in I$, then closures, we obtain $\mathfrak{C} \subseteq \mathfrak{D}$.

Since $\mathfrak{C}=\operatorname{cl}_{\mathbf{B}}(C), C$ is included in $\mathfrak{D}$, too, and that means that $C+r \subseteq$ $c l_{\mathbf{B}}\left(C^{\prime}+r^{\prime}\right)$. By Lemma 5.7, item 3, $(C, r) \leq d_{\mathcal{H}}^{+}\left(C^{\prime}, r^{\prime}\right)$, so $(C, r)$ is the least upper bound of $\left(C_{i}, r_{i}\right)_{i \in I}$.

\subsection{Algebraicity}

We will show that if $X, d$ is algebraic complete, then so is $\mathcal{H}_{0} X, d_{\mathcal{H}}$. In order to show that, we will need the following, rather miraculous, result: when $X, d$ is continuous complete, for every $a>0, \mathcal{L}_{\alpha}(X, d)$, with the subspace topology from $\mathcal{L} X$, is stably compact [11, Lemma 7.3, item 4]; more- 
over, for every finite family of center points $x_{1}, \ldots, x_{n}$ of $X, d$, the maps $h \mapsto \max _{i=1}^{n} h\left(x_{i}\right)$ and $h \mapsto \min _{i=1}^{n} h\left(x_{i}\right)$ are continuous from $\mathcal{L}_{\alpha}(X, d)^{\mathrm{d}}$ to $\left(\overline{\mathbb{R}}_{+}\right)^{\mathrm{d}}[11$, Corollary 7.7 , item 2]. (When $n=0$, we take the max to be 0 , and the min to be $+\infty$.) We use the notation $Y^{\mathrm{d}}$, for a stably compact space $Y$, to denote its de Groot dual: a space with the same points, and whose closed sets are the compact saturated subsets of $Y$. See Chapter 9 of [7] for stably compact spaces. It will be enough to know that, given a stably compact space $Y$, with specialization ordering $\leq$, we can build its patch space $Y^{\text {patch }}$, which is $Y$ with the coarsest topology containing all the open sets of $Y$ and of $Y^{\mathrm{d}}$, and that $Y^{\text {patch }}$ is compact Hausdorff. $\overline{\mathbb{R}}_{+}$, with its Scott topology, is stably compact; the open subsets of $\overline{\mathbb{R}}_{+}^{\mathrm{d}}$ are the intervals $\left[0, r\left[, r \in \mathbb{R}_{+}\right.\right.$, plus the whole space; and $\overline{\mathbb{R}}_{+}^{\text {patch }}$ is $\overline{\mathbb{R}}_{+}$with its usual Hausdorff topology.

Lemma 5.16. Let $x_{1}, \ldots, x_{n}$ be finitely many center points in a continuous complete quasi-metric space $X, d, F$ be a prevision on $X$, and $a \in \mathbb{R}_{+}$. The sets $\left\{h \in \mathcal{L}_{1}(X, d) \mid \max _{j=1}^{n} h\left(x_{j}\right)<F(h)+a\right\}$ and $\left\{h \in \mathcal{L}_{1}(X, d) \mid\right.$ $\left.\min _{j=1}^{n} h\left(x_{j}\right)<F(h)+a\right\}$ are open in $\left(\mathcal{L}_{1}(X, d)\right)^{\text {patch }}$.

Proof. Let $V$ be the first of those sets (the case of the other one is dealt with similarly), $f$ be the map $h \mapsto \max _{i=1}^{n} h\left(x_{i}\right)$, and $g$ be the map $h \mapsto F(h)+a$. For each $h \in \mathcal{L}_{1}(X, d), h$ is in $V$ if and only if there is a non-negative real number $r$ such that $f(h)<r$ and $r<g(h)$, so $V=\bigcup_{r \in \mathbb{R}_{+}} f^{-1}([0, r[) \cap$ $\left.\left.g^{-1}(] r,+\infty\right]\right)$. Now $f^{-1}\left(\left[0, r[)\right.\right.$ is open in $\left(\mathcal{L}_{1}(X, d)\right)^{\mathrm{d}}$ since $f$ is continuous from $\mathcal{L}_{\alpha}(X, d)^{\mathrm{d}}$ to $\left(\overline{\mathbb{R}}_{+}\right)^{\mathrm{d}}$ for any $\alpha>0$, as we have recalled above. Hence it is also open in $\left(\mathcal{L}_{1}(X, d)\right)^{\text {patch }}$. Additionally, $\left.\left.g^{-1}(] r,+\infty\right]\right)$ is open in $\mathcal{L}_{1}(X, d)$, because $g$ is Scott-continuous and the topology on $\mathcal{L}_{1}(X, d)$ is the subspace topology from $\mathcal{L} X$ (whose topology is the Scott topology). Hence $\left.\left.g^{-1}(] r,+\infty\right]\right)$ is also open in $\left(\mathcal{L}_{1}(X, d)\right)^{\text {patch }}$.

Lemma 5.17. Let $X, d$ be a continuous complete quasi-metric space. For all center points $x_{1}, \ldots, x_{n}, \downarrow\left\{x_{1}, \cdots, x_{n}\right\}$ is a center point of $\mathcal{H}_{0} X, d_{\mathcal{H}}$, and also of $\mathcal{H} X, d_{\mathcal{H}}$ if $n \geq 1$.

Proof. Let $C_{0} \stackrel{\text { def }}{=} \downarrow\left\{x_{1}, \cdots, x_{n}\right\}$. For every $h \in \mathcal{L} X, F^{C_{0}}(h)=\max _{j=1}^{n} h\left(x_{j}\right)$, where the maximum is taken to be 0 if $n=0$. Let $U \stackrel{\text { def }}{=} B_{\left(F^{\left.C_{0}, 0\right),<\epsilon}\right.}^{d_{\mathrm{R}}^{+}} \cdot U$ is upwards-closed: if $\left(F^{C}, r\right) \leq d_{\mathrm{KR}}^{+}\left(F^{C^{\prime}}, r^{\prime}\right)$ and $\left(F^{C}, r\right) \in U$, then $d_{\mathrm{KR}}\left(F^{C}, F^{C^{\prime}}\right) \leq$ $r-r^{\prime}$ and $d_{\mathrm{KR}}\left(F^{C_{0}}, F^{C}\right)<\epsilon-r$, so $d_{\mathrm{KR}}\left(F^{C_{0}}, F^{C^{\prime}}\right)<\epsilon-r^{\prime}$ by the triangular inequality, and that means that $\left(F^{C^{\prime}}, r^{\prime}\right)$ is in $U$. 
In order to show that $U$ is Scott-open, we consider a directed family $\left(C_{i}, r_{i}\right)_{i \in I}$ in $\mathbf{B}\left(\mathcal{H}_{0} X, d_{\mathcal{H}}\right)\left(\operatorname{resp} ., \mathbf{B}\left(\mathcal{H} X, d_{\mathcal{H}}\right)\right)$ with supremum $(C, r)$, and we assume that $\left(F^{C}, r\right)$ is in $U$. By Proposition 5.12, directed suprema of formal balls are naive suprema, so $r=\inf _{i \in I} r_{i}$ and $C$ is characterized by the fact that, for every $h \in \mathcal{L}_{1}(X, d), F^{C}(h)=\sup _{i \in I}\left(F^{C_{i}}(h)+r-r_{i}\right)$. Since $\left(F^{C}, r\right)$ is in $U, d_{\mathrm{KR}}\left(F^{C_{0}}, F^{C}\right)<\epsilon-r$, so $\epsilon>r$ and $F^{C_{0}}(h)-\epsilon+r<F^{C}(h)$. Therefore, for every $h \in \mathcal{L}_{1}(X, d)$, there is an index $i \in I$ such that $F^{C_{0}}(h)-\epsilon+r<$ $F^{C_{i}}(h)+r-r_{i}$, or equivalently:

$$
\max _{j=1}^{n} h\left(x_{j}\right)<F^{C_{i}}(h)+\epsilon-r_{i} .
$$

Moreover, since $\epsilon>r=\inf _{i \in I} r_{i}$, we may assume that $i$ is so large that $\epsilon>r_{i}$.

Let $V_{i}$ be the set of all $h \in \mathcal{L}_{1}(X, d)$ satisfying (6). By Lemma 5.16, $V_{i}$ is open in $\left(\mathcal{L}_{1}(X, d)\right)^{\text {patch }}$. The latter is compact (and Hausdorff), and we have just argued that $\left(V_{i}\right)_{i \in I}$ is an open cover of that space. Hence we can extract a finite subcover $\left(V_{i}\right)_{i \in J}$ : for every $h \in \mathcal{L}_{1}(X, d)$, there is an index $i$ in the finite set $J$ such that (6) holds. By directedness, one can require that $i$ be the same for all $h$. This shows that $\left(F^{C_{i}}, r_{i}\right)$ is in $U$, proving the claim.

Remark 5.18. A similar result holds for $d_{\mathcal{H}}^{a}$ instead of $d_{\mathcal{H}}, a \in \mathbb{R}_{+}, a>0$ : on a continuous complete quasi-metric space $X, d$, and for all center points $x_{1}, \ldots, x_{n}, \downarrow\left\{x_{1}, \cdots, x_{n}\right\}$ is a center point of $\mathcal{H}_{0} X, d_{\mathcal{H}}^{a}$, and also of $\mathcal{H} X, d_{\mathcal{H}}^{a}$ if $n \geq 1$. The proof is as for Lemma $5.1 \%$.

A strong basis of a standard quasi-metric space $X, d$ is any set $\mathcal{B}$ of center points of $X$ such that, for every $x \in X,(x, 0)$ is the supremum of a directed family of formal balls with center points in $\mathcal{B}$ (see Definition 7.4.66 of [7], which is stated with $d$-finite points and Cauchy nets instead of center points and directed families of formal balls). The standard space $X, d$ is algebraic if and only if it has a strong basis. The largest strong basis is simply the set of all center points.

Lemma 5.19. Let $X, d$ be a standard algebraic quasi-metric space, with strong basis $\mathcal{B}$. For every $\epsilon>0$, the open balls $B_{x,<r}^{d}$ with $x \in \mathcal{B}$ and $0<r<\epsilon$ form a base of the d-Scott topology on $X$.

Proof. We recall that every standard algebraic quasi-metric space is continuous, so $\mathbf{B}(X, d)$ is a continuous poset. Let $U$ be any open subset of $X$, 
and $y \in U$. Then $(y, 0)$ is in $\widehat{U} \cap V_{\epsilon}$, and is the supremum of a directed family of formal balls of the form $(x, r)$ with $x \in \mathcal{B}$ and $(x, r) \ll(y, 0)$. Hence one of them is in $\widehat{U} \cap V_{\epsilon}$. Since it is in $V_{\epsilon}, r<\epsilon$, and since $(x, r) \ll(y, 0)$ where $x$ is a center point in a standard algebraic space, $d(x, y)<r$, so $y$ is in $B_{x,<r}^{d}$. Moreover, $B_{x,<r}^{d}$ is open, still because $x$ is a center point, and because $B_{x,<r}^{d}=B_{(x, 0),<r}^{d^{+}} \cap X$. Finally, $B_{x,<r}^{d}$ is included in $\uparrow(x, r) \cap X \subseteq \widehat{U} \cap X=U$.

We will prove the algebraicity of Hoare hyperspaces by relying on the following lemma. We will reuse it for Smyth hyperspaces, as well as in Part IV.

Lemma 5.20. Let $Y, \partial$ be a standard quasi-metric space, $\mathcal{B}_{0}$ be a set of center points of $Y, \partial$, and let also $\tau$ be a topology on the set $Y$, satisfying the following assumptions:

(i) every open ball centered at any point of $\mathcal{B}_{0}$ is $\tau$-open;

(ii) for every $\epsilon>0$, for every $\tau$-open neighborhood $U$ of $z$, there is an open ball $B_{y,<r}^{\partial}$ included in $U$ and which contains $z$, for some $y \in \mathcal{B}_{0}$ and some $0<r \leq \epsilon$;

(iii) for all $y, z \in Y$, if $y \leq z$ in the specialization preordering $\leq$ of $\tau$, then $y \leq^{\partial} z$, namely $\partial(y, z)=0$.

Then $Y, \partial$ is algebraic, $\mathcal{B}_{0}$ is a strong basis, and $\tau$ coincides with the $\partial$-Scott topology.

Conditions (i) and (ii) say that, for every $\epsilon>0$, the open balls $B_{y,<r}^{\partial}$ with $y \in \mathcal{B}_{0}$ and $0<r \leq \epsilon$ form a base of the topology $\tau$. We prefer to make those conditions explicit, as this is in this form that we will use them.

Proof. Let $z$ be any point of $Y$. We need to show that $(z, 0)$ is the supremum of some directed family $D$ of formal balls with centers in $\mathcal{B}_{0}$. We let $D$ be the family of formal balls $(y, r)$ with $y \in \mathcal{B}_{0}$ such that $\partial(y, z)<r$, or equivalently such that $z \in B_{y,<r}^{\partial}$.

By assumption (ii) with $U \stackrel{\text { def }}{=} Y$ and $\epsilon$ arbitrary, $D$ is non-empty. We also note that $D$ contains formal balls $(y, r)$ with arbitrarily small radii $r$.

We claim that $D$ is directed. Let $\left(y_{1}, r_{1}\right)$ and $\left(y_{2}, r_{2}\right)$ be two elements of $D$. We have $\partial\left(y_{1}, z\right)<r_{1}$ and $\partial\left(y_{2}, z\right)<r_{2}$. Let $\epsilon$ be some number such 
that $0<\epsilon<\min \left(r_{1}, r_{2}\right)$ and $\partial\left(y_{1}, z\right)<r_{1}-\epsilon, \partial\left(y_{2}, z\right)<r_{2}-\epsilon$. Then $U \stackrel{\text { def }}{=} B_{y_{1},<r_{1}-\epsilon}^{\partial} \cap B_{y_{2},<r_{2}-\epsilon}^{\partial}$ is a $\tau$-open neighborhood of $z$, by assumption (i). By assumption (ii), there is an open ball $B_{y,<r}^{\partial}$ included in $U$, and which contains $z$, for some $y \in \mathcal{B}_{0}$ and $0<r \leq \epsilon$. In particular, $(y, r)$ is in $D$. Since $y$ is in $B_{y_{1},<r_{1}-\epsilon}^{\partial}, \partial\left(y_{1}, y\right)<r_{1}-\epsilon$. Since $r \leq \epsilon, \partial\left(y_{1}, y\right)<r_{1}-r$, so $\left(y_{1}, r_{1}\right) \leq \partial^{+}(y, r)$. Similarly, $\left(y_{2}, r_{2}\right) \leq \partial^{+}(y, r)$. Therefore $D$ is directed.

For every $(y, r) \in D, \partial(y, z)<r$, so $(y, r) \leq^{\partial^{+}}(z, 0)$. Hence $(z, 0)$ is an upper bound of $D$. We claim that it is the least upper bound of $D$. Let $\left(z^{\prime}, s\right)$ be any upper bound of $D$. Since $D$ contains formal balls of arbitrarily small radii, $s$ must be equal to 0 . For every $\tau$-open neighborhood $U$ of $z$, by assumption (ii) there is an open ball $B_{y,<r}^{\partial}$ included in $U$ and which contains $z$, for some $y \in \mathcal{B}_{0}$. Then $\partial(y, z)<r$, so $\partial(y, z)<r-\epsilon$ for some $\epsilon$ such that $0<\epsilon<r$. It follows that $(y, r-\epsilon)$ is in $D$. Since $\left(z^{\prime}, 0\right)$ is an upper bound of $D,(y, r-\epsilon) \leq \partial^{+}\left(z^{\prime}, 0\right)$, so that $\partial\left(y, z^{\prime}\right) \leq r-\epsilon<r$. It follows that $z^{\prime}$ is in $B_{y,<r}^{\partial}$, hence in $U$. We have just shown that every $\tau$-open neighborhood of $z$ contains $z^{\prime}$, so $z \leq z^{\prime}$. By assumption (iii), $\partial\left(z, z^{\prime}\right)=0$, so $(z, 0) \leq \partial^{+}\left(z^{\prime}, 0\right)$.

Finally, we show that $\tau$ coincides with the $\partial$-Scott topology. We have just shown that $\mathcal{B}_{0}$ is a strong basis. By Lemma 5.19, the open balls $B_{y,<r}^{d}$ with $y \in \mathcal{B}_{0}$ form a base of the $\partial$-Scott topology on $Y$. By assumptions (i) and (ii) they also form a base of the topology $\tau$, so $\tau$ coincides with the $\partial$-Scott topology.

Lemma 5.6 of [12] states that, for any standard algebraic space $X, d$, with strong basis $\mathcal{B}, \mathbf{B}(X, d)$ is a continuous depo, with a basis consisting of the formal balls $(x, r)$ with $x \in \mathcal{B}$; and that, moreover, for every $x \in \mathcal{B}$, $(x, r) \ll(y, s)$ if and only if $d(x, y)<r-s$. This implies that, given any open neighborhood $V$ of any formal ball $(y, s)$ in $\mathbf{B}(X, d)$, there is a formal ball $(x, r)$ in $V$ such that $x \in \mathcal{B}$ and such that $(x, r) \ll(y, s)$, namely such that $d(x, y)<r-s$.

Lemma 5.21. Let $X, d$ be a standard quasi-metric space, and $C_{0} \stackrel{\text { def }}{=} \downarrow\left\{x_{1}, \cdots\right.$, $\left.x_{n}\right\}$, where each $x_{i}$ is a center point of $X, d$. For every $r>0$, the subsets $B_{C_{0},<r}^{d_{\mathcal{H}}}$ and $\bigcap_{i=1}^{n} \diamond B_{x_{i},<r}^{d}$ of $\mathcal{H}_{0} X$ (resp., $\mathcal{H} X$ if $n \geq 1$ ) coincide. Similarly with $d_{\mathcal{H}}^{a}(a>0)$ in place of $d_{\mathcal{H}}$, provided that $r \leq a$.

Proof. For every $r>0, B_{C_{0},<r}^{d_{\mathcal{H}}}$ is the set of closed subsets (resp., nonempty) $C$ such that $d_{\mathcal{H}}\left(C_{0}, C\right)<r$, equivalently such that $d\left(x_{i}, C^{\prime}\right)<r$ for every $i, 1 \leq i \leq n$. By Fact 5.2 and since each $x_{i}$ is a center point, 
$d\left(x_{i}, C^{\prime}\right)$ is equal to $\inf _{y \in C^{\prime}} d\left(x_{i}, y\right)$. Therefore, $d\left(x_{i}, C^{\prime}\right)<r$ is equivalent to the existence of a point $y_{i} \in C^{\prime}$ such that $d\left(x_{i}, y_{i}\right)<r$, hence to the fact that $C^{\prime}$ intersects $B_{x_{i},<r}^{d}$. It follows that $B_{C_{0},<r}^{d_{\mathcal{H}}}=\bigcap_{i=1}^{n} \diamond B_{x_{i},<r}^{d}$.

Given any $a>0$, we recall from Remark 5.9 that $d_{\mathcal{H}}^{a}\left(C_{0}, C\right)=\min (a$, $\left.d_{\mathcal{H}}\left(C_{0}, C\right)\right)$, so that, if $r \leq a, B_{C_{0},<r}^{d_{\mathcal{H}}^{a}}=B_{C_{0},<r}^{d_{\mathcal{H}}}$.

Theorem 5.22 (Algebraicity of Hoare hyperspaces). Let $X, d$ be an algebraic complete quasi-metric space, with strong basis $\mathcal{B}$. The spaces $\mathcal{H}_{0} X, d_{\mathcal{H}}$ and $\mathcal{H} X, d_{\mathcal{H}}$ are algebraic complete.

Every closed set of the form $\downarrow\left\{x_{1}, \cdots, x_{n}\right\}$ where every $x_{i}$ is a center point (and with $n \geq 1$ in the case of $\mathcal{H} X$ ) is a center point of $\mathcal{H}_{0} X, d_{\mathcal{H}}$ (resp., $\left.\mathcal{H} X, d_{\mathcal{H}}\right)$, and those form a strong basis, even when each $x_{i}$ is taken from $\mathcal{B}$. The $d_{\mathcal{H}}$-Scott topology coincides with the lower Vietoris topology.

Proof. $\mathcal{H}_{0} X, d_{\mathcal{H}}$ and $\mathcal{H} X, d_{\mathcal{H}}$ are complete by Proposition 5.12 , and $\downarrow\left\{x_{1}, \cdots\right.$, $\left.x_{n}\right\}$ is a center point as soon as every $x_{i}$ is a center point, by Lemma 5.17. We let $\mathcal{B}_{0}$ be set of all center points of the form $\downarrow\left\{x_{1}, \cdots, x_{n}\right\}$ where each $x_{i}$ is in $\mathcal{B}$, and $\tau$ be the lower Vietoris topology on $\mathcal{H}_{0} X$, resp. $\mathcal{H} X$. The conclusion will follow from Lemma 5.20, provided we verify its assumptions.

(i) Let $C_{0} \stackrel{\text { def }}{=} \downarrow\left\{x_{1}, \cdots, x_{n}\right\}$ where $x_{1}, \ldots, x_{n}$ are in $\mathcal{B}$. Then $B_{C_{0},<r}^{d_{\mathcal{H}}}$ is equal to the lower Vietoris open set $\bigcap_{i=1}^{n} \diamond B_{x_{i},<r}^{d}$ by Lemma 5.21.

(ii) Let $\epsilon>0, C \in \mathcal{H}_{0} X$ (resp., $\mathcal{H} X$ ), and $\mathcal{U}$ be a lower Vietoris open neighborhood of $C$. There are finitely many open subsets $U_{1}, \ldots, U_{n}$ of $X$ such that $C \in \bigcap_{i=1}^{n} \diamond U_{i} \subseteq \mathcal{U}$. For each $i, 1 \leq i \leq n, C$ intersects $U_{i}$, say at $y_{i}$. By Lemma 5.6 of [12] (recalled right before Lemma 5.21), $\left(y_{i}, 0\right)$ is the supremum of a directed family of formal balls in $\mathbf{B}(X, d)$, with centers in $\mathcal{B}$, all of those formal balls being way below $\left(y_{i}, 0\right)$. One of them, call it $\left(z_{i}, t_{i}\right)$, is in the Scott-open set $\widehat{U}_{i}$. Since $\left(z_{i}, t_{i}\right) \ll\left(y_{i}, 0\right)$ and since $z_{i}$ is a center point, by the same Lemma 5.6 we have $d\left(z_{i}, y_{i}\right)<t_{i}$. Let $r$ be such that $0<r<\epsilon$ and $d\left(z_{i}, y_{i}\right)<t_{i}-r$ for every $i, 1 \leq i \leq n$. Hence $\left(z_{i}, t_{i}-r\right) \ll\left(y_{i}, 0\right)$. We repeat the same argument as the one that just allowed us to find $\left(z_{i}, t_{i}\right)$ : there is a formal ball $\left(x_{i}, r_{i}\right) \ll\left(y_{i}, 0\right)$ in the Scott-open set $\uparrow\left(z_{i}, t_{i}-r\right) \cap V_{r}$ such that $x_{i}$ is in $\mathcal{B}$.

Since $\left(x_{i}, r_{i}\right) \ll\left(y_{i}, 0\right)$ and since $x_{i}$ is a center point, $d\left(x_{i}, y_{i}\right)<r_{i}$, and since $\left(x_{i}, r_{i}\right) \in V_{r}, r_{i}<r$. Therefore $y_{i}$ is in $B_{x_{i},<r}^{d}$. Since $y_{i}$ is in $C$ for each $i, C$ is in $\bigcap_{i=1}^{n} \diamond B_{x_{i},<r}^{d}$. By Lemma 5.21, the latter is equal to $B_{C_{0},<r}^{d_{\mathcal{H}}}$, where $C_{0} \stackrel{\text { def }}{=} \downarrow\left\{x_{1}, \cdots, x_{n}\right\}$. 
It remains to show that $B_{C_{0},<r}^{d_{\mathcal{H}}}=\bigcap_{i=1}^{n} \diamond B_{x_{i},<r}^{d}$ is included in $\bigcap_{i=1}^{n} \diamond U_{i}$, hence in $\mathcal{U}$. In order to do so, it is enough to prove that, for every $i$ with $1 \leq i \leq n, B_{x_{i},<r}^{d}$ is included in $U_{i}$. Let $z$ be any point of $B_{x_{i},<r}^{d}$. Then $d\left(x_{i}, z\right)<r$. Since $\left(x_{i}, r_{i}\right)$ is in $\uparrow\left(z_{i}, t_{i}-r\right)$, and since $z_{i}$ is a center point, $d\left(z_{i}, x_{i}\right)<t_{i}-r-r_{i} \leq t_{i}-r$, so $d\left(z_{i}, z\right)<t_{i}-r+r=t_{i}$. It follows that $\left(z_{i}, t_{i}\right) \ll(z, 0)$, and since $\left(z_{i}, t_{i}\right)$ is in $\widehat{U}_{i}$, so is $(z, 0)$. Therefore $z$ is in $U_{i}$.

(iii) The specialization preordering $\leq$ of the lower Vietoris topology is inclusion. This is well-known, but here is a quick proof nonetheless. If $C \leq C^{\prime}$ then $C \in \diamond U$ implies $C^{\prime} \in \diamond U$, where $U$ is the complement of $C^{\prime}$, so $C \subseteq C^{\prime}$. Conversely, if $C \subseteq C^{\prime}$, then $C \in \bigcap_{i=1}^{n} \diamond U_{i}$ means that $C$ intersects every $U_{i}$, hence $C^{\prime}$ does, too, so that $C^{\prime}$ is in $\bigcap_{i=1}^{n} \diamond U_{i}$. We conclude since $\leq^{d_{\mathcal{H}}}$ is also the inclusion ordering, by Lemma 5.7, item 2 .

Remark 5.23. The same result holds for $d_{\mathcal{H}}^{a}$, for every $a \in \mathbb{R}_{+}, a>0$ : when $X, d$ is algebraic complete, $\mathcal{H}_{0} X, d_{\mathcal{H}}^{a}$ and $\mathcal{H} X, d_{\mathcal{H}}^{a}$ are algebraic complete, with the same strong basis, and the $d_{\mathcal{H}}^{a}$-Scott topology coincides with the lower Vietoris topology. The proof is the same, except for the following points. In (i), the argument only applies if $r \leq a$; when $r>a, B_{C_{0},<r}^{d_{\mathcal{H}}^{a}}$ is simply the whole space. In (ii), we choose $r$ such that $r<\min (a, \epsilon)$, not just $r<\epsilon$, in order to ensure that $\bigcap_{i=1}^{n} \diamond B_{x_{i},<r}^{d}$ is equal to $B_{C_{0},<r}^{d_{\mathcal{H}}^{a}}$.

\subsection{Continuity}

We can now deduce the continuous case from the algebraic case. The key is that the continuous complete quasi-metric spaces are exactly the 1Lipschitz continuous retracts of algebraic complete quasi-metric spaces [13, Theorem 7.9]. A 1-Lipschitz continuous retraction of $Y, \partial$ onto $X, d$ is a pair of two 1-Lipschitz continuous maps $r: Y, \partial \rightarrow X, d$ (itself called the retraction) and $s: X, d \rightarrow Y, \partial$ (the section) such that $r \circ s=\mathrm{id}_{X}$. If that is the case, then $X, d$ is a 1-Lipschitz continuous retract of $Y, \partial$.

Writing $f[C]$ for the image of $C$ by $f$, we have the following.

Lemma 5.24. Let $X, d$ and $Y, \partial$ be two continuous complete quasi-metric spaces, and $f: X, d \mapsto Y, \partial$ be a 1-Lipschitz continuous map. The map $\mathcal{H} f: \mathcal{H}_{0} X, d_{\mathcal{H}} \rightarrow \mathcal{H}_{0} Y, d_{\mathcal{H}}$ defined by $\mathcal{H} f(C) \stackrel{\text { def }}{=} c l(f[C])$ is 1-Lipschitz continuous. Moreover, $F^{\mathcal{H} f(C)}=\mathbf{P} f\left(F^{C}\right)$ for every $C \in \mathcal{H}_{0} X$.

Similarly with $\mathcal{H}$ instead of $\mathcal{H}_{0}$, or with $d_{\mathcal{H}}^{a}$ instead of $d_{\mathcal{H}}$. 
Proof. We first check that $F^{\mathcal{H} f(C)}=\mathbf{P} f\left(F^{C}\right)$. For every $h \in \mathcal{L} X, F^{\mathcal{H} f(C)}(h)=$ $\sup _{y \in c l(f[C])} h(y)=\sup _{y \in f[C]} h(y)$ by Lemma 5.10. That is equal to $\sup _{x \in X} h(f(x))$. We also have $\mathbf{P} f\left(F^{C}\right)(h)=F^{C}(h \circ f)=\sup _{x \in X} h(f(x))$. Therefore $F^{\mathcal{H} f(C)}=$ $\mathbf{P} f\left(F^{C}\right)$, which shows that the isometry of Proposition 5.8 is natural.

By Lemma 4.21, $\mathbf{P} f$ is 1-Lipschitz, so $\mathbf{B}^{1}(\mathbf{P} f)$ is monotonic. Also, $\mathbf{P} f$ maps discrete sublinear previsions to discrete sublinear previsions, and normalized previsions to normalized previsions. By Proposition 5.12, $\mathcal{H}_{0} X, d_{\mathcal{H}}$ and $\mathcal{H} X, d_{\mathcal{H}}$ are complete, hence through the isometry of Proposition 5.8, the corresponding spaces of discrete sublinear previsions are complete as well. Moreover, directed suprema of formal balls are computed as naive suprema. By Lemma 4.22, $\mathbf{B}^{1}(\mathbf{P} f)$ preserves naive suprema, hence all directed suprema. It must therefore be Scott-continuous, and using the (natural) isometry of Proposition 5.8, $\mathbf{B}^{1}(\mathcal{H} f)$ must also be Scott-continuous. Hence $\mathcal{H} f$ is 1-Lipschitz continuous.

In the case of $d_{\mathcal{H}}^{a}$, the argument is the same, except that we use Remark 5.13 instead of Proposition 5.12.

Let $X, d$ be a continuous complete quasi-metric space. We have noted that $X, d$ is a 1-Lipschitz continuous retract of some algebraic complete quasimetric space $Y, \partial$. Let $r$ be the retraction and $s$ be the section.

By Lemma 5.24, $\mathcal{H}_{0} r$ and $\mathcal{H}_{0} s$ are also 1-Lipschitz continuous. Also, $\mathcal{H}_{0} r \circ \mathcal{H}_{0} s=\operatorname{id}_{\mathcal{H}_{0} X}$ : through the isometry $C \mapsto F^{C}$, that boils down to $\mathbf{P} r \circ \mathbf{P} s=$ id, which is easily checked since $\mathbf{P} r\left(\mathbf{P} s\left(F^{C}\right)\right)(h)=\mathbf{P} s\left(F^{C}\right)(h \circ r)=$ $F^{C}(h \circ r \circ s)=F^{C}(h)$, for all $C$ and $h$. Therefore $\mathcal{H}_{0} X, d_{\mathcal{H}}$ is a 1-Lipschitz continuous retract of $\mathcal{H}_{0} Y, \partial_{\mathcal{H}}$. (Similarly with $d_{\mathcal{H}}^{a}$ and $\partial_{\mathcal{H}}^{a}$.) Theorem 5.22 states that $\mathcal{H}_{0} Y, \partial_{\mathcal{H}}$ and $\mathcal{H} Y, \partial_{\mathcal{H}}$ (resp., $\partial_{\mathcal{H}}^{a}$, using Remark 5.23 instead) is algebraic complete, whence:

Theorem 5.25 (Continuity for Hoare hyperspaces). Let $X, d$ be a continuous complete quasi-metric space. The quasi-metric spaces $\mathcal{H}_{0} X, d_{\mathcal{H}}$ and $\mathcal{H}_{0} X, d_{\mathcal{H}}^{a}\left(a \in \mathbb{R}_{+}, a>0\right)$ are continuous complete. Similarly with $\mathcal{H} X$.

Together with Lemma 5.24, and Theorem 5.22 for the algebraic case, we obtain the following.

Corollary 5.26. $\mathcal{H}_{0}, d_{\mathcal{H}}$ defines an endofunctor on the category of continuous complete quasi-metric spaces and 1-Lipschitz continuous map. Similarly with $\mathcal{H}$ instead of $\mathcal{H}_{0}$, with $d_{\mathcal{H}}^{a}$ instead of $d_{\mathcal{H}}(a>0)$, or with algebraic instead of continuous. 


\subsection{The lower Vietoris topology}

We will show that, when $X, d$ is continuous complete, the $d_{\mathrm{KR}}$-Scott topology on $\mathcal{H}_{0} X$ coincides with the weak topology. We will first use the following general result on spaces of previsions. We recall that the sets $[h>a]=\{F \mid F(h)>a\}$ form a subbase of the weak topology, where $a \in \mathbb{R}_{+}$and $h \in \mathcal{L} X$. Since $h$ is the supremum of the chain of maps $h^{(\alpha)}$, $\alpha \in \mathbb{R}_{+} \backslash\{0\},[h>a]=\bigcup_{\alpha \in \mathbb{R}_{+} \backslash\{0\}}\left[h^{(\alpha)}>a\right]=\bigcup_{\alpha \in \mathbb{R}_{+} \backslash\{0\}}\left[1 / \alpha . h^{(\alpha)}>a / \alpha\right]$. Since $1 / \alpha \cdot h^{(\alpha)}$ is 1 -Lipschitz continuous, the sets $[h>b]$ with $h \in \mathcal{L}_{1}(X, d)$, now, and $b \in \mathbb{R}_{+}$, form a smaller subbase of the weak topology. The proof of the following is exactly as for Proposition 9.1 of [12].

Proposition 5.27. Let $X, d$ be a standard quasi-metric space, $a, a^{\prime}>0$ with $a \leq a^{\prime}$. Let $S$ be a subset of the following properties on previsions: sublinearity, superlinearity, linearity, subnormalization, normalization, discreteness; and let $Y$ denote the set of previsions on $X$ satisfying $S$.

Assume finally that the spaces $Y, d_{K R}^{a}, Y, d_{K R}^{a^{\prime}}$ and $Y, d_{K R}$ are Yonedacomplete and that directed suprema in their spaces of formal balls are computed as naive suprema.

Then we have the following inclusions of topologies on $Y$ :

$$
\text { weak } \subseteq d_{K R^{-}}^{a} S \operatorname{cott} \subseteq d_{K R^{\prime}}^{a^{\prime}} S \operatorname{cott} \subseteq d_{K R^{-}} \text {Scott }
$$

Proof. First inclusion. We use yet another topology, this time on $\mathbf{B}\left(Y, d_{\mathrm{KR}}^{a}\right)$. Let $[h>b]^{+}$be defined as the set of those formal balls $(F, r)$ with $F \in Y$ such that $F(h)>r+b$. The weak $k^{+}$topology on $\mathbf{B}\left(Y, d_{\mathrm{KR}}^{a}\right)$ has a subbase of open sets given by the sets $[h>b]^{+}$, for every $h \in \mathcal{L}_{1}^{a}(X, d)$ and $b \in \mathbb{R}_{+}$.

$[h>b]^{+}$is upwards-closed in $\mathbf{B}\left(Y, d_{\mathrm{KR}}^{a}\right)$. Indeed, assume that $(G, r) \in$ $[h>b]^{+}$, where $h \in \mathcal{L}_{1}^{a}(X, d)$, and that $(G, r) \leq d_{\mathrm{KR}}^{a+}\left(G^{\prime}, r^{\prime}\right)$. By Lemma 4.13, item $2, G(h)-r \leq G^{\prime}(h)-r^{\prime}$. Since $G(h)>r+b, G^{\prime}(h)>r^{\prime}+b$, namely $G^{\prime} \in[h>b]^{+}$.

In order to show that $[h>b]^{+}$is Scott-open in $\mathbf{B}\left(Y, d_{\mathrm{KR}}^{a}\right)$, let $\left(G_{i}, r_{i}\right)_{i \in I}$ be a directed family with (naive) supremum $(G, r)$ in $\mathbf{B}\left(Y, d_{\mathrm{KR}}^{a}\right)$ and assume that $(G, r) \in[h>b]^{+}$. Since $G(h)=\sup _{i \in I}\left(G_{i}(h)+r-r_{i}\right)>r+b, G_{i}(h)>r_{i}+b$ for some $i \in I$, i.e., $\left(G_{i}, r_{i}\right)$ is in $[h>b]^{+}$.

We can now proceed to show that $[h>b]$ is $d_{\mathrm{KR}}^{a}$-Scott open, for every $h \in \mathcal{L}_{1}^{a}(X, d)$. Equating $Y$ with the subset of all formal balls $(G, 0), G \in Y$, $[h>b]$ is equal to $Y \cap[h>b]^{+}$. Since $[h>b]^{+}$is Scott-open, $[h>b]$ is $d_{\mathrm{KR}}^{a}$-Scott open. 
Second and third inclusions. The proofs of the second and third inclusions are similar. We rely on the easily checked inequalities $d_{\mathrm{KR}}^{a} \leq d_{\mathrm{KR}}^{a^{\prime}} \leq d_{\mathrm{KR}}$, for $a \leq a^{\prime}$. This implies that: $(*)(F, r) \leq d_{\mathrm{KR}}^{+}\left(F^{\prime}, s\right)$ implies $(F, r) \leq d_{\mathrm{KR}}^{a^{\prime}+}\left(F^{\prime}, s\right)$, and that $(F, r) \leq d_{\mathrm{KR}}^{a^{\prime}+}\left(F^{\prime}, s\right)$ implies $(F, r) \leq d_{\mathrm{KR}}^{a+}\left(F^{\prime}, s\right)$.

Let $\mathcal{U}$ be a Scott-open subset of $\mathbf{B}\left(Y, d_{\mathrm{KR}}^{a}\right)$. Since $\mathcal{U}$ is upwards-closed in $\leq d_{\mathrm{KR}}^{a+}$, it is also upwards-closed in $\leq d_{\mathrm{KR}}^{a^{\prime}+}$ by $(*)$. Assume now that $(F, r) \in \mathcal{U}$ is the supremum in $\mathbf{B}\left(Y, d_{\mathrm{KR}}^{a^{\prime}}\right)$ of a family $\left(F_{i}, r_{i}\right)_{i \in I}$ that is directed with respect to $\leq d_{\mathrm{KR}}^{a^{\prime}+}$. By $(*)$ again, $\left(F_{i}, r_{i}\right)_{i \in I}$ is directed with respect to $\leq d_{\mathrm{KR}}^{a+}$. Therefore $\left(F_{i}, r_{i}\right)_{i \in I}$ has a supremum $\left(F^{\prime}, r^{\prime}\right)$ in $\mathbf{B}\left(Y, d_{\mathrm{KR}}^{a}\right)$ that is uniquely characterized by $r^{\prime} \stackrel{\text { def }}{=} \inf _{i \in I} r_{i}, F^{\prime}(h) \stackrel{\text { def }}{=} \sup _{i \in I}\left(F_{i}(h)+r-r_{i}\right)$ for every $h \in \mathcal{L}_{1}^{a}(X, d)$, since suprema are assumed to be naive. However, by naivety again, this time applied to the definition of $(F, r), r=\inf _{i \in I} r_{i}$ and $F(h)=$ $\sup _{i \in I}\left(F_{i}(h)+r-r_{i}\right)$ for every $h \in \mathcal{L}_{1}^{a^{\prime}}(X, d)$. This holds in particular for every $h \in \mathcal{L}_{1}^{a}(X, d)$, so $F=F^{\prime}$. We have therefore obtained that $(F, r)$ is also the supremum of the directed family $\left(F_{i}, r_{i}\right)_{i \in I}$ in $\mathbf{B}\left(Y, d_{\mathrm{KR}}^{a}\right)$. Since $(F, r) \in \mathcal{U}$, some $\left(F_{i}, r_{i}\right)$ is also in $\mathcal{U}$ since $\mathcal{U}$ is Scott-open in $\mathbf{B}\left(Y, d_{\mathrm{KR}}^{a}\right)$. This shows that $\mathcal{U}$ is $\operatorname{Scott-open~in~} \mathbf{B}\left(Y, d_{\mathrm{KR}}^{a^{\prime}}\right)$. Similarly, we show that every Scott-open subset of $\mathbf{B}\left(Y, d_{\mathrm{KR}}^{a^{\prime}}\right)$ is Scott-open in $\mathbf{B}\left(Y, d_{\mathrm{KR}}\right)$.

By taking intersections $\mathcal{U} \cap Y$, it follows that every $d_{\mathrm{KR}}^{a}$-Scott open is $d_{\mathrm{KR}}^{a^{\prime}}$-Scott open, and that every $d_{\mathrm{KR}}^{a^{\prime}}$-Scott open is $d_{\mathrm{KR}}$-Scott open.

Considering Lemma 3.4, Remark 5.9, and Proposition 5.12, Proposition 5.27 then entails that, if $X, d$ is standard and Lipschitz regular, or continuous complete, then:

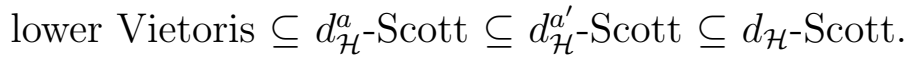

We can say more if we focus on continuous complete quasi-metric spaces. The following fact is justified by the equality $\mathbf{P} f^{-1}([h>b])=[h \circ f>b]$.

Fact 5.28. Let $S$ be any subset of the following properties on previsions: sublinearity, superlinearity, linearity, subnormalization, normalization, discreteness. For every continuous map $f: X \rightarrow Y, \mathbf{P} f$ is continuous from the space of all previsions on $X$ satisfying $S$ to the space of all previsions on $Y$ satisfying $S$, both spaces being given the weak topology.

We also observe the following easy fact.

Fact 5.29. If $A$ is a space with two topologies $\mathcal{O}_{1}$ and $\mathcal{O}_{2}$, and both embed into a topological space $B$ by the same topological embedding, then $\mathcal{O}_{1}=\mathcal{O}_{2}$. 
Theorem 5.30 ( $d_{\mathcal{H}}$ quasi-metrizes the lower Vietoris topology). Let $X, d$ be a continuous complete quasi-metric space. The $d_{\mathcal{H}}$-Scott topology, the $d_{\mathcal{H}}^{a}$-Scott topology, for every $a \in \mathbb{R}_{+}, a>0$, and the lower Vietoris topology all coincide on $\mathcal{H}_{0} X$, resp. $\mathcal{H} X$.

Proof. $X, d$ is the 1-Lipschitz continuous retract of an algebraic complete quasi-metric space $Y, \partial$. Let us call $s: X \rightarrow Y$ the section and $r: Y \rightarrow X$ the retraction. Using Proposition 5.8, we confuse $\mathcal{H}_{0} X$ with the corresponding space of discrete sublinear previsions, and similarly for $\mathcal{H} X, \mathcal{H}_{0} Y, \mathcal{H} Y$. Then $\mathbf{P} s$ and $\mathbf{P} r$ form a 1-Lipschitz continuous section-retraction pair by Lemma 4.21, and in particular $\mathbf{P} s$ is an embedding of $\mathcal{H}_{0} X$ into $\mathcal{H}_{0} Y$ with their $d_{\mathcal{H}}$-Scott topologies (similarly with $\mathcal{H}$, or with $d_{\mathcal{H}}^{a}$ in place of $d_{\mathcal{H}}$ ). However, $s$ and $r$ are also just continuous, since 1-Lipschitz continuous maps between standard quasi-metric spaces are continuous. Hence $\mathbf{P} s$ and $\mathbf{P} r$ also form a section-retraction pair between the same spaces, this time with their weak topologies (as spaces of previsions), by Fact 5.28, that is, with their lower Vietoris topologies, by Lemma 3.4. Since $Y, \partial$ is algebraic complete, we know from Theorem 5.22 and Remark 5.23 that the two topologies on $\mathcal{H}_{0} Y$ (resp., $\mathcal{H} Y$ ) are the same. Fact 5.29 then implies that the two topologies on $\mathcal{H}_{0} X$ (resp., $\left.\mathcal{H} X\right)$ are the same as well.

\section{The Smyth Hyperspace}

\subsection{The $d_{\mathcal{Q}}$ Quasi-Metric}

Our developments concerning the Smyth hyperspace $\mathcal{Q} X$ are almost entirely parallel to our study of the Hoare hyperspace $\mathcal{H} X$. However, instead of defining a specific quasi-metric on such subsets, as we did with $d_{\mathcal{H}}$ on the Hoare hyperspace, we shall reuse $d_{\mathrm{KR}}$, on the isomorphic space of discrete superlinear previsions on $X$.

Definition $6.1\left(d_{\mathcal{Q}}, d_{\mathcal{Q}}^{a}\right)$. Let $X, d$ be a quasi-metric space. For any two non-empty compact saturated subsets $Q, Q^{\prime}$ of $X$, let $d_{\mathcal{Q}}\left(Q, Q^{\prime}\right) \stackrel{\text { def }}{=} d_{K R}\left(F_{Q}, F_{Q^{\prime}}\right)$. We also let $d_{\mathcal{Q}}^{a}\left(Q, Q^{\prime}\right) \stackrel{\text { def }}{=} d_{K R}^{a}\left(F_{Q}, F_{Q^{\prime}}\right)$, for every $a>0$.

We give a more concrete description of $d_{\mathcal{Q}}$ in Lemma 6.3. We start with an easy observation.

Lemma 6.2. Let $X, d$ be a standard quasi-metric space. For every point $x^{\prime} \in X$, the map $d\left({ }_{-}, x^{\prime}\right)$ is in $\mathcal{L}_{1}(X, d)$. 
Proof. Let $h \stackrel{\text { def }}{=} d\left({ }_{-}, x^{\prime}\right)$. This is a 1-Lipschitz continuous map [7, Exercise 7.4.36], hence it is 1-Lipschitz continuous, since $X, d$ is standard. Alternatively, $h$ is also equal to the map $d\left({ }_{-}, C\right)$ where $C$ is the closed set $\downarrow x^{\prime}$, and that is 1-Lipschitz continuous by Fact 5.1.

Lemma 6.3. Let $X, d$ be a standard quasi-metric space. For all non-empty compact saturated subsets $Q, Q^{\prime}, d_{\mathcal{Q}}\left(Q, Q^{\prime}\right)=\sup _{x^{\prime} \in Q^{\prime}} d\left(Q, x^{\prime}\right)$, where $d\left(Q, x^{\prime}\right) \stackrel{\text { def }}{=}$ $\inf _{x \in Q} d\left(x, x^{\prime}\right)$.

Proof. We first show that $d_{\mathcal{Q}}\left(Q, Q^{\prime}\right) \leq \sup _{x^{\prime} \in Q^{\prime}} d\left(Q, x^{\prime}\right)$. It suffices to show that for every $r \in \mathbb{R}_{+}$such that $r<d_{\mathcal{Q}}\left(Q, Q^{\prime}\right), r \leq d\left(Q, x^{\prime}\right)$ for some $x^{\prime} \in Q^{\prime}$. Since $r<d_{\mathcal{Q}}\left(Q, Q^{\prime}\right)=d_{\mathrm{KR}}\left(F_{Q}, F_{Q^{\prime}}\right)$, there is an $h \in \mathcal{L}_{1}(X, d)$ such that $F_{Q}(h)>F_{Q^{\prime}}(h)+r$. Let $x^{\prime} \in Q^{\prime}$ be such that $F_{Q^{\prime}}(h)=h\left(x^{\prime}\right)$, using Proposition 3.5, item 1. For every $x \in Q, h(x)>h\left(x^{\prime}\right)+r$, so, since $h$ is 1-Lipschitz, $d\left(x, x^{\prime}\right)>r$. It follows that $d\left(Q, x^{\prime}\right) \geq r$.

In the reverse direction, we fix $x^{\prime} \in Q^{\prime}, r<d\left(Q, x^{\prime}\right)$, and we show that there is an $h \in \mathcal{L}_{1}(X, d)$ such that $F_{Q}(h) \geq r+F_{Q^{\prime}}(h)$. Since $r<d\left(Q, x^{\prime}\right)$, $d\left(x, x^{\prime}\right)>r$ for every $x \in Q$. Let $h \stackrel{\text { def }}{=} d\left({ }_{-}, x^{\prime}\right)$. This is in $\mathcal{L}_{1}(X, d)$ by Lemma 6.2, and $h(x)>r$ for every $x \in Q$. It follows that $F_{Q}(h)=$ $\min _{x \in Q} h(x)>r$, whereas $F_{Q^{\prime}}(h) \leq h\left(x^{\prime}\right)=0$.

Lemma 6.3 means that $d_{\mathcal{Q}}\left(Q, Q^{\prime}\right)$ is given by one half of the familiar Hausdorff formula $d_{\mathcal{Q}}\left(Q, Q^{\prime}\right)=\sup _{x^{\prime} \in Q^{\prime}} \inf _{x \in Q} d\left(x, x^{\prime}\right)$. The quasi-metric $d_{\mathcal{H}}$ is given by a formula that is almost the other half, $\sup _{x \in Q} \inf _{x^{\prime} \in Q^{\prime}} d\left(x, x^{\prime}\right)$.

Remark 6.4. We also have $d_{\mathcal{Q}}^{a}\left(Q, Q^{\prime}\right)=\min \left(a, d_{\mathcal{Q}}\left(Q, Q^{\prime}\right)\right)$, for every $a \in$ $\mathbb{R}_{+}, a>0$. In one direction, $d_{\mathcal{Q}}^{a}\left(Q, Q^{\prime}\right)=d_{K R}^{a}\left(F_{Q}, F_{Q^{\prime}}\right) \leq a$ and $d_{\mathcal{Q}}^{a}\left(Q, Q^{\prime}\right)=$ $d_{K R}^{a}\left(F_{Q}, F_{Q^{\prime}}\right) \leq d_{K R}\left(F_{Q}, F_{Q^{\prime}}\right)=d_{\mathcal{Q}}\left(Q, Q^{\prime}\right), \operatorname{sod}_{\mathcal{Q}}^{a}\left(Q, Q^{\prime}\right) \leq \min \left(a, d_{\mathcal{Q}}\left(Q, Q^{\prime}\right)\right)$. In the other direction, we fix $r<\min \left(a, d_{\mathcal{Q}}\left(Q, Q^{\prime}\right)\right)$. In particular, $r<a$ and there is an $x^{\prime} \in Q^{\prime}$ such that $r<d\left(Q, x^{\prime}\right)$, whence $d\left(x, x^{\prime}\right)>r$ for every $x \in$ $Q$. Let $h \stackrel{\text { def }}{=} \min \left(a, d\left({ }_{-}, x^{\prime}\right)\right) \in \mathcal{L}_{1}^{a}(X, d): F_{Q}(h)=\min \left(a, \min _{x \in Q} d\left(x, x^{\prime}\right)\right)>$ $r$, and $F_{Q^{\prime}}(h)=0$, so $d_{\mathcal{Q}}^{a}\left(Q, Q^{\prime}\right)=d_{K R}^{a}\left(F_{Q}, F_{Q^{\prime}}\right) \geq r$. Since $r$ is arbitrary, $\min \left(a, d_{\mathcal{Q}}\left(Q, Q^{\prime}\right)\right) \leq d_{\mathcal{Q}}^{a}\left(Q, Q^{\prime}\right)$.

Lemma 6.5. Let $X, d$ be a standard quasi-metric space. For every nonempty compact saturated subset $Q$ of $X$, for every $x^{\prime} \in X$, the following hold:

1. there is an $x \in Q$ such that $d\left(Q, x^{\prime}\right)=d\left(x, x^{\prime}\right) ; d\left(Q, x^{\prime}\right)=\min _{x \in Q} d\left(x, x^{\prime}\right)$; 
2. $d\left(Q, x^{\prime}\right)=0$ if and only if $x^{\prime} \in Q$;

3. $d\left(Q, x^{\prime}\right) \leq d\left(Q, y^{\prime}\right)+d\left(y^{\prime}, x^{\prime}\right)$ for every $y^{\prime} \in X$.

Proof. 1. Because $d\left({ }_{-}, x^{\prime}\right)$ is in $\mathcal{L}_{1}(X, d) \subseteq \mathcal{L} X$ (Lemma 6.2), it reaches its minimum on the compact set $Q$.

2. Let $x \in Q$ be such that $d\left(Q, x^{\prime}\right)=d\left(x, x^{\prime}\right)$, by item 1 . If $d\left(Q, x^{\prime}\right)=0$, then $d\left(x, x^{\prime}\right)=0$, so $x \leq x^{\prime}$, and since $Q$ is saturated, $x^{\prime}$ is in $Q$. Conversely, if $x^{\prime} \in Q$, then $d\left(x^{\prime}, x^{\prime}\right)=0$ implies that $d\left(Q, x^{\prime}\right)=0$.

3. Let $y^{\prime}$ be an arbitrary point of $X$. For every $x \in Q, d\left(x, x^{\prime}\right) \leq$ $d\left(x, y^{\prime}\right)+d\left(y^{\prime}, y\right)$, and we obtain the result by taking minima over $x \in Q$ on both sides.

Lemma 6.6. Let $X, d$ be a standard quasi-metric space. For all non-empty compact saturated subsets $Q, Q^{\prime}$ of $X, d_{\mathcal{Q}}\left(Q, Q^{\prime}\right)=0$ if and only if $Q \supseteq Q^{\prime}$.

Proof. $d_{\mathcal{Q}}\left(Q, Q^{\prime}\right)=0$ if and only if $\sup _{x^{\prime} \in Q^{\prime}} d\left(Q, x^{\prime}\right)=0$ by Lemma 6.3 , if and only if $d\left(Q, x^{\prime}\right)=0$ for every $x^{\prime} \in Q^{\prime}$. By Lemma 6.5, this is equivalent to requiring that $x^{\prime} \in Q$ for every $x^{\prime} \in Q^{\prime}$.

Remark 6.7. Because of Remark 6.4, it also follows that for every $a \in \mathbb{R}_{+}$, $a>0, d_{\mathcal{Q}}^{a}\left(Q, Q^{\prime}\right)=0$ if and only if $Q \supseteq Q^{\prime}$.

\subsection{Completeness}

Lemma 6.8. Let $X, d$ be a standard quasi-metric space. For every compact saturated subset $\mathcal{Q}$ of $\mathbf{B}(X, d)$ such that $F_{\mathcal{Q}}$ is supported on $V_{1 / 2^{n}}$ for every $n \in \mathbb{N}$,

1. $\mathcal{Q}$ is included in $X$;

2. $\mathcal{Q}$ is a compact saturated subset of $X$;

3. $F_{\mathcal{Q}}$ is supported on $X$.

ProOF. If $\mathcal{Q}$ is empty, this is obvious, so let us assume that $\mathcal{Q}$ is non-empty.

For any two real numbers $r, s>0, \chi_{V_{r}}$ and $\chi_{V_{s}}$ coincide on $V_{1 / 2^{n}}$, where $n$ is any natural number large enough that $1 / 2^{n} \leq r, s$. Therefore $F_{\mathcal{Q}}\left(\chi_{V_{r}}\right)=$ $F_{\mathcal{Q}}\left(\chi_{V_{s}}\right)$. It follows that $F_{\mathcal{Q}}\left(\chi_{V_{r}}\right)$ is a value $a$ that does not depend on $r>0$. The union $\bigcup_{r \in \mathbb{R}_{+} \backslash\{0\}} V_{r}$ is the whole space of formal balls, so $\sup _{r \in \mathbb{R}_{+} \backslash\{0\}} \chi_{V_{r}}=1$. Since $F_{\mathcal{Q}}$ is Scott-continuous, $\sup _{r \in \mathbb{R}_{+} \backslash\{0\}} F_{\mathcal{Q}}\left(\chi_{V_{r}}\right)=$ 
$F_{\mathcal{Q}}(\mathbf{1})=1$ (since $\mathcal{Q}$ is non-empty), and that is also equal to $\sup _{r \in \mathbb{R}_{+} \backslash\{0\}} a=a$. We have obtained that $F_{\mathcal{Q}}\left(\chi_{V_{r}}\right)=1$ for every $r>0$, namely that $\mathcal{Q} \subseteq V_{r}$ for every $r>0$. As a consequence $\mathcal{Q}$ is included in $\bigcap_{r>0} V_{r}=X$, showing item 1 . Item 2 follows from the easily checked fact that a compact saturated subset $\mathcal{Q}$ of a subset $X$ of a space $Y$ is also compact saturated in $X$, seen as a subspace. Item 3 is now obvious.

By Proposition 3.5, if $X$ is sober in its $d$-Scott topology, then the bijection $Q \mapsto F_{Q}$ is an isometry of $\mathcal{Q} X, d_{\mathcal{Q}}$ onto the set of normalized discrete superlinear previsions on $X$, with the usual $d_{\mathrm{KR}}$ quasi-metric.

Proposition 6.9 (Completeness, Smyth hyperspace). Let $X, d$ be a sober standard quasi-metric space. Then $\mathcal{Q} X, d_{\mathcal{Q}}$ is complete.

Suprema of directed families of formal balls of previsions are naive suprema.

PRoOF. This follows from Proposition 4.25, since the assumption on supports is verified by Lemma 6.8.

Remark 6.10. Similarly, when $X, d$ is standard and sober, $\mathcal{Q} X, d_{\mathcal{Q}}^{a}$ is complete for every $a \in \mathbb{R}_{+}, a>0$.

As a first example of sober standard quasi-metric spaces, one can find all metric spaces. They are all standard, and they are sober since Hausdorff. They are also continuous, and even algebraic, because every point is a center point in a metric space.

A second family of sober standard quasi-metric spaces consists in the continuous complete quasi-metric spaces, in particular all algebraic complete quasi-metric spaces. We have already mentioned that all complete quasimetric spaces are standard [13, Proposition 2.2]; that continuous complete quasi-metric spaces are sober is Proposition 4.1 of [13]. Sobriety could be dispensed with it we worked directly on normalized discrete superlinear previsions, instead of the more familiar elements of $\mathcal{Q} X$.

Remark 6.11. Sobriety cannot be dispensed with in Proposition 6.9. In order to see this, recall that every poset $X$ can be seen as a quasi-metric space, by defining $d_{\leq}(x, y)$ as 0 if $x \leq y$, $+\infty$ otherwise. Then $X, d_{\leq}$is standard. It is complete as a quasi-metric space if and only if it is a dcpo [13, Example 1.6]. Using Lemma 6.3, one can check that $d_{\mathcal{Q}}$ is $d_{\supseteq}$, so that $\mathcal{Q} X, d_{\mathcal{Q}}$ is a complete quasi-metric space if and only if $\mathcal{Q} X$ is a dcpo under ․ Now consider Johnstone's space $\mathbb{J}$, a famous counterexample in domain 
theory [14]. We will not describe it here. It suffices to know that its points are pairs $(m, n)$ where $m \in \mathbb{N}$ and $n \in \mathbb{N} \cup\{\omega\}$, that it is a dcpo, and that $Q_{A} \stackrel{\text { def }}{=}\{(m, \omega) \mid m \in A\}$ is an element of $\mathcal{Q}(\mathbb{J})$ for every non-empty subset $A$ of $\mathbb{J}\left[7\right.$, Exercise 8.3.9]. Then the elements $Q_{\{n, n+1, \cdots\}}, n \in \mathbb{N}$, form a chain without any upper bound in $\mathcal{Q}(\mathbb{J})$, showing that $\mathbb{J}, d_{\leq}$is a complete quasi-metric space whose Smyth hyperspace is not complete under $d_{\leq \mathcal{Q}}$.

\section{3. $d_{\mathcal{Q}}$-Limits}

As for Lemma 5.15, there is a more direct expression of directed suprema of formal balls over $\mathcal{Q} X, d_{\mathcal{Q}}$, i.e., of $d_{\mathcal{Q}}$-limits, than by relying on naive suprema. Recall that $Q+r$ is the set $\{(x, r) \mid x \in Q\}$.

We will use the following results. First, we define the radius $r(\mathcal{Q})$ of a non-empty compact saturated subset $\mathcal{Q}$ of $\mathcal{B}(X, d)$ as $\sup \{r \mid(x, r) \in \mathcal{Q}\}$. When $X, d$ is standard, $(x, r) \mapsto r$ is continuous from $\mathbf{B}(X, d)$ to $\left(\overline{\mathbb{R}}_{+}\right)^{o p}$, so this supremum is reached; in particular, $r(\mathcal{Q})<+\infty$.

Then, we need the following, from [2]. An LCS-complete space is any space that is homeomorphic to a $G_{\delta}$ subset of a locally compact sober space $Y$. A typical example is given by continuous complete quasi-metric space $X$ in their $d$-Scott topology [2, Theorem 4.1, together with Proposition 3.3], since $X, d$ embeds as a $G_{\delta}$ subset in the continuous dcpo $Y=\mathbf{B}(X, d)$. By $[2$, Theorem 17.4, Remark 17.1], the non-empty compact saturated subsets of $X$ are exactly the filtered intersections $\bigcap_{i \in I} \mathcal{Q}_{i}$ of non-empty compact saturated subsets $\mathcal{Q}_{i}$ of $\mathbf{B}(X, d)$ such that $\inf _{i \in I} r\left(\mathcal{Q}_{i}\right)=0$.

We will also use the fact that every sober space is well-filtered, meaning that given any filtered family $\left(Q_{i}\right)_{i \in I}$ of compact saturated subsets and any open subset $U$, if $\bigcap_{i \in I} Q_{i} \subseteq U$ then for some $i \in I, Q_{i} \subseteq U[7$, Proposition 8.3.5].

Lemma 6.12. Let $X, d$ be a sober standard quasi-metric space. Then:

1. In $\mathbf{B}\left(\mathcal{Q} X, d_{\mathcal{Q}}\right),(Q, r) \leq{ }^{d_{\mathcal{Q}}^{+}}\left(Q^{\prime}, r^{\prime}\right)$ if and only if $Q^{\prime}+r^{\prime} \subseteq \uparrow_{\mathbf{B}}(Q+r)$, where $\uparrow_{\mathbf{B}}$ is upward closure in $\mathbf{B}(X, d)$.

2. If $X, d$ is continuous complete, then for every directed family $\left(Q_{i}, r_{i}\right)_{i \in I}$, the supremum $(Q, r)$ is given by $r=\inf _{i \in I} r_{i}$ and $Q=\bigcap_{i \in I} \uparrow_{\mathbf{B}}\left(Q_{i}+r_{i}-\right.$ $r)$.

Proof. 1. If $(Q, r) \leq{ }^{d_{\mathcal{Q}}^{+}}\left(Q^{\prime}, r^{\prime}\right)$, then $d_{\mathcal{Q}}\left(Q, Q^{\prime}\right) \leq r-r^{\prime}$, so $r \geq r^{\prime}$ and for every $x^{\prime} \in Q^{\prime}, d\left(Q, x^{\prime}\right) \leq r-r^{\prime}$, by Lemma 6.3. Using Lemma 6.5, item 1 , 
there is an $x \in Q$ such that $d\left(x, x^{\prime}\right) \leq r-r^{\prime}$. In particular, $(x, r) \leq^{d^{+}}\left(x^{\prime}, r^{\prime}\right)$. This shows that every element of $Q^{\prime}+r^{\prime}$ is in $\uparrow_{\mathbf{B}}(Q, r)$.

Conversely, if every element of $Q^{\prime}+r^{\prime}$ is in $\uparrow_{\mathbf{B}}(Q, r)$, then in particular $r \geq r^{\prime}$. Indeed, since $Q^{\prime}$ is non-empty, we can find $x^{\prime} \in Q^{\prime}$, and $\left(x^{\prime}, r^{\prime}\right) \in$ $\uparrow_{\mathbf{B}}(Q, r)$. There is an $x \in Q$ such that $(x, r) \leq d^{+}\left(x^{\prime}, r^{\prime}\right)$, and that implies $r \geq r^{\prime}$.

We rewrite the inclusion $Q^{\prime}+r^{\prime} \subseteq \uparrow_{\mathbf{B}}(Q+r)$ as: for every $x^{\prime} \in Q^{\prime}$, there is an $x \in Q$ such that $(x, r) \leq d^{+}\left(x^{\prime}, r^{\prime}\right)$, i.e., $d\left(x, x^{\prime}\right) \leq r-r^{\prime}$. It follows that $d_{\mathcal{Q}}\left(Q, Q^{\prime}\right) \leq r-r^{\prime}$ by Lemma 6.3 , whence $(Q, r) \leq d_{\mathcal{Q}}^{+}\left(Q^{\prime}, r^{\prime}\right)$.

2. Let $r \stackrel{\text { def }}{=} \inf _{i \in I} r_{i}$ and $Q \stackrel{\text { def }}{=} \bigcap_{i \in I} \uparrow_{\mathbf{B}}\left(Q_{i}+r_{i}-r\right)$. Since $X, d$ is standard, the map ${ }_{-}+r_{i}-r$ is Scott-continuous for every $i \in I$, and since $\eta_{X}$ is also continuous, the image of $Q_{i}+r_{i}-r$ of $Q_{i}$ by their composition is compact in $\mathbf{B}(X, d)$. Hence $\uparrow_{\mathbf{B}}\left(Q_{i}+r_{i}-r\right)$ is compact saturated in $\mathbf{B}(X, d)$.

Let $i \sqsubseteq j$ if and only if $\left(Q_{i}, r_{i}\right) \leq d_{\mathcal{Q}}^{+}\left(Q_{j}, r_{j}\right)$. If $i \sqsubseteq j$ then $\left(Q_{i}, r_{i}-r\right) \leq d_{\mathcal{Q}}^{+}$ $\left(Q_{j}, r_{j}-r\right)$, so $\uparrow_{\mathbf{B}}\left(Q_{i}+r_{i}-r\right) \supseteq \uparrow_{\mathbf{B}}\left(Q_{j}+r_{j}-r\right)$ by item 1 . It follows that the family $\left(\uparrow_{\mathbf{B}}\left(Q_{i}+r_{i}-r\right)\right)_{i \in I}$ is filtered, with respect to inclusion.

The radius of $\uparrow_{\mathbf{B}}\left(Q_{i}+r_{i}-r\right)$, as defined above, is equal to $r_{i}-r$, and $\inf _{i \in I}\left(r_{i}-r\right)=0$. Therefore, by the result cited above, $Q$ is non-empty, compact, and saturated in $X$, hence an element of $\mathcal{Q} X$.

We claim that $\uparrow_{\mathbf{B}}(Q+r)=\bigcap_{i \in I} \uparrow_{\mathbf{B}}\left(Q_{i}+r_{i}\right)$. For every element $(x, r)$ of $Q+r$ (i.e., $x \in Q$ ), for every $i \in I,(x, 0)$ lies above some element $\left(x_{i}, r_{i}-r\right)$ where $x_{i} \in Q_{i}$; so $d\left(x_{i}, x\right) \leq r_{i}-r$, which implies $\left(x_{i}, r_{i}\right) \leq{ }^{d^{+}}(x, r)$, hence $(x, r) \in \uparrow_{\mathbf{B}}\left(Q_{i}+r_{i}\right)$. That shows $Q+r \subseteq \bigcap_{i \in I} \uparrow_{\mathbf{B}}\left(Q_{i}+r_{i}\right)$. Since the right-hand side is upwards-closed, $\uparrow_{\mathbf{B}}(Q+r)$ is also included in $\bigcap_{i \in I} \uparrow_{\mathbf{B}}\left(Q_{i}+r_{i}\right)$. In the reverse direction, it is enough to show that every Scott-open neighborhood $\mathcal{U}$ of $\uparrow_{\mathbf{B}}(Q+r)$ contains $\bigcap_{i \in I} \uparrow_{\mathbf{B}}\left(Q_{i}+r_{i}\right)$. Since $X, d$ is standard, the map $+r$ is Scott-continuous, so $\left({ }_{-}+r\right)^{-1}(\mathcal{U})$ is Scott-open. Since $Q+r$ is included in $\mathcal{U}$, $Q$ is included in $(-+r)^{-1}(\mathcal{U})$. Since $X, d$ is continuous complete, $\mathbf{B}(X, d)$ is a continuous dcpo, hence is sober, hence well-filtered, so, using the definition of $Q, Q_{i}+r_{i}-r$ is included in $\left({ }_{-}+r\right)^{-1}(\mathcal{U})$ for some $i \in I$. That means that $Q_{i}+r_{i}$ is included in $\mathcal{U}$, hence also $\uparrow_{\mathbf{B}}\left(Q_{i}+r_{i}\right)$ since every Scott-open set is upwards-closed. Therefore, $\mathcal{U}$ indeed contains $\bigcap_{i \in I} \uparrow_{\mathbf{B}}\left(Q_{i}+r_{i}\right)$.

We can now finish the proof. Since $Q+r$ is included in $\uparrow_{\mathbf{B}}\left(Q_{i}+r_{i}\right)$, $\left(Q_{i}, r_{i}\right) \leq d_{\mathcal{Q}}^{+}(Q, r)$ by item 1 . For every upper bound $\left(Q^{\prime}, r^{\prime}\right)$ of $\left(Q_{i}, r_{i}\right)_{i \in I}$, $r^{\prime} \leq \inf _{i \in I} r_{i}=r$, and $Q^{\prime}+r^{\prime}$ is included in $\uparrow_{\mathbf{B}}\left(Q_{i}+r_{i}\right)$ for every $i \in I$, by item 1. Hence $Q^{\prime}+r^{\prime}$ is included in $\bigcap_{i \in I} \uparrow_{\mathbf{B}}\left(Q_{i}+r_{i}\right)=\uparrow_{\mathbf{B}}(Q+r)$, showing that $(Q, r) \leq d_{\mathcal{Q}}^{+}\left(Q^{\prime}, r^{\prime}\right)$, by item 1 again. 
Remark 6.13. One can rephrase Lemma 6.12, item 2, as follows. Let $X, d$ be a continuous complete quasi-metric space. Let $\left(Q_{i}\right)_{i \in I, \sqsubset}$ be a Cauchyweightable net in $\mathcal{Q} X, d_{\mathcal{Q}}$, and $\left(Q_{i}, r_{i}\right)_{i \in I, \sqsubset}$ be some corresponding Cauchyweighted net. Then the $d_{\mathcal{Q}}$-limit of $\left(Q_{i}\right)_{i \in I, \sqsubset}$ is $Q$ is the filtered intersection $\bigcap_{i \in I} \uparrow_{\mathbf{B}}\left(Q_{i}+r_{i}\right)$. Since $Q$ is included in $X$, this is also equal to $\bigcap_{i \in I}(X \cap$ $\left.\uparrow_{\mathbf{B}}\left(Q_{i}+r_{i}\right)\right)$. Note that $X \cap \uparrow_{\mathbf{B}}\left(Q_{i}+r_{i}\right)$ is the set $Q_{i}^{+r_{i}}$ of points $\{x \in X \mid$ $\left.\exists y \in Q_{i} . d(y, x) \leq r_{i}\right\}$ that are at distance at most $r_{i}$ from $Q_{i}$. (Beware that there is no reason to believe that $Q_{i}^{+r_{i}}$ would be compact.) Hence $Q$ is the filtered intersection $\bigcap_{i \in I} Q_{i}^{+r_{i}}$.

\subsection{Algebraicity}

The following is proved just like Lemma 5.17.

Lemma 6.14. Let $X, d$ be a continuous complete quasi-metric space. For all $n \geq 1$, and center points $x_{1}, \ldots, x_{n}, \uparrow\left\{x_{1}, \cdots, x_{n}\right\}$ is a center point of $\mathcal{Q} X, d_{\mathcal{Q}}$.

Proof. First, we recall that every continuous complete quasi-metric space is sober.

Let $Q_{0} \stackrel{\text { def }}{=} \uparrow\left\{x_{1}, \cdots, x_{n}\right\}$. For every $h \in \mathcal{L} X, F_{Q_{0}}(h)=\min _{j=1}^{n} h\left(x_{j}\right)$. Let $U \stackrel{\text { def }}{=} B_{\left(F_{Q_{0}}, 0\right),<\epsilon}^{d_{\mathrm{KR}}^{+}} . \quad U$ is upwards-closed: if $\left(F_{Q}, r\right) \leq d_{\mathrm{KR}}^{+}\left(F_{Q^{\prime}}, r^{\prime}\right)$ and $\left(F_{Q}, r\right)$ is in $U$, then $d_{\mathrm{KR}}\left(F_{Q}, F_{Q^{\prime}}\right) \leq r-r^{\prime}$, and $d_{\mathrm{KR}}\left(F_{Q_{0}}, F_{Q}\right)<\epsilon-r$, so $d_{\mathrm{KR}}\left(F_{Q_{0}}, F_{Q^{\prime}}\right)<r-r^{\prime}+\epsilon-r=\epsilon-r^{\prime}$, showing that $\left(F_{Q^{\prime}}, r^{\prime}\right)$ is in $U$.

In order to show that $U$ is Scott-open, we consider a directed family $\left(Q_{i}, r_{i}\right)_{i \in I}$ in $\mathbf{B}\left(\mathcal{Q} X, d_{\mathcal{Q}}\right)$, with supremum $(Q, r)$, and we assume that $\left(F_{Q}, r\right)$ is in $U$. By Proposition 6.9, this is a naive supremum, so $r=\inf _{i \in I} r_{i}$ and $Q$ is characterized by the fact that, for every $h \in \mathcal{L}_{1}(X, d), F_{Q}(h)=$ $\sup _{i \in I}\left(F_{Q_{i}}(h)+r-r_{i}\right)$. Since $\left(F_{Q}, r\right)$ is in $U, d_{\mathrm{KR}}\left(F_{Q_{0}}, F_{Q}\right)<\epsilon-r$, so $\epsilon>r$ and $F_{Q_{0}}(h)-\epsilon+r<F_{Q}(h)$ for every $h \in \mathcal{L}_{1}(X, d)$. Therefore, for every $h \in \mathcal{L}_{1}(X, d)$, there is an index $i \in I$ such that $F_{Q_{0}}(h)-\epsilon+r<F_{Q_{i}}(h)+r-r_{i}$, or equivalently:

$$
\min _{j=1}^{n} h\left(x_{j}\right)<F_{Q_{i}}(h)+\epsilon-r_{i} .
$$

Moreover, since $\epsilon>r=\inf _{i \in I} r_{i}$, we may assume that $i$ is so large that $\epsilon>r_{i}$.

Let $V_{i}$ be the set of all $h \in \mathcal{L}_{1}(X, d)$ such that $(7)$ holds. Each $V_{i}$ is open in $\mathcal{L}_{1}(X, d)^{\text {patch }}$ by Lemma 5.16. Hence $\left(V_{i}\right)_{i \in I}$ is an open cover of $\mathcal{L}_{1}(X, d)^{\text {patch }}$. The latter is a compact (Hausdorff) space. Hence we can extract a finite subcover $\left(V_{i}\right)_{i \in J}$ : for every $h \in \mathcal{L}_{1}(X, d)$, there is an index $i$ in the finite set 
$J$ such that (7) holds. By directedness, one can require that $i$ be the same for all $h$. This shows that $\left(F_{Q_{i}}, r_{i}\right)$ is in $U$, proving the claim.

Remark 6.15. A similar result holds for $\mathcal{Q} X, d_{\mathcal{Q}}^{a}$ for any $a \in \mathbb{R}_{+}, a>0$, and the argument is the same as for Lemma 6.14.

Lemma 6.16. Let $X, d$ be a standard algebraic quasi-metric space, with strong basis $\mathcal{B}$. For every compact subset $Q$ of $X$, for every open neighborhood $U$ of $Q$, and for every $\epsilon>0$, there are finitely many points $x_{1}, \ldots$, $x_{n}$ in $\mathcal{B}$ and radii $0<r_{1}, \ldots, r_{n}<\epsilon$ such that $Q \subseteq \bigcup_{j=1}^{n} B_{x_{j},<r_{j}}^{d} \subseteq U$.

Proof. Every $y \in Q$ has an open neighborhood of the form $B_{x,<r}^{d}$ with $x \in \mathcal{B}$ and $0<r<\epsilon$ by Lemma 5.19. We then extract a finite subcover, using the compactness of $Q$.

Lemma 6.17. Let $X, d$ be a standard quasi-metric space. Let $Q$ be a compact saturated subset of $X$. For all center points $x_{1}, \ldots, x_{m}$ and for every $r>$ 0 such that $Q \subseteq \bigcup_{j=1}^{m} B_{x_{j},<r}^{d}$, there is an $\epsilon>0, \epsilon<r$, such that $Q \subseteq$ $\bigcup_{j=1}^{m} B_{x_{j},<r-\epsilon}^{d}$.

ProOF. For each $\epsilon>0, \epsilon<r$, we consider the open subset $U_{\epsilon} \stackrel{\text { def }}{=} \bigcup_{j=1}^{m} B_{x_{j},<r-\epsilon}^{d}$. (We recall that $B_{x_{j},<r-\epsilon}^{d}$ is open, since it is equal to $B_{\left(x_{j}, 0\right),<r-\epsilon}^{d^{+}} \cap X$, which is Scott-open since $x_{j}$ is a center point.) For every $x \in Q, x$ is in some open ball $B_{x_{j},<r}^{d}$ by assumption, so $d\left(x, x_{j}\right)<r$. This implies that there is an $\epsilon>0$ such that $d\left(x, x_{j}\right)<r-\epsilon$ (in particular $\left.\epsilon<r\right)$, hence that $x \in U_{\epsilon}$. The family $\left(U_{\epsilon}\right)_{0<\epsilon<r}$ is then a chain that forms an open cover of $Q$. Since $Q$ is compact, $Q$ is included in $U_{\epsilon}$ for some $\epsilon>0$ with $\epsilon<r$.

Lemma 6.18. Let $X, d$ be a standard quasi-metric space, and $Q_{0} \stackrel{\text { def }}{=} \uparrow\left\{x_{1}, \cdots\right.$, $\left.x_{n}\right\}$, where $n \geq 1$ and each $x_{i}$ is a center point of $X, d$. For every $r>0$, $B_{Q_{0},<r}^{d_{\mathcal{Q}}}=\square\left(\bigcup_{i=1}^{n} B_{x_{i},<r}^{d}\right)$. Similarly with $d_{\mathcal{Q}}^{a}(a>0)$ in place of $d_{\mathcal{Q}}$, provided that $r \leq a$.

ProOf. For every $Q \in B_{Q_{0},<r}^{d_{\mathcal{Q}}}$, we have $d_{\mathcal{Q}}\left(Q_{0}, Q\right)<r$, so using Lemma 6.3, for every element $y$ of $Q$, there is an $x \in Q_{0}$ such that $d(x, y)<r$. Given such an $x$, we have $x_{i} \leq x$ for some $i, 1 \leq i \leq n$, so $d\left(x_{i}, y\right) \leq d\left(x_{i}, x\right)+d(x, y)<$ $0+r=r$. This shows that every $y \in Q$ is in the open ball $B_{x_{i},<r}^{d}$ for some $i$, hence that $Q \in \square\left(\bigcup_{i=1}^{n} B_{x_{i},<r}^{d}\right)$. Conversely, for every $Q \in \square\left(\bigcup_{i=1}^{n} B_{x_{i},<r}^{d}\right)$, 
we show that $Q$ is in $B_{Q_{0},<r}^{d_{\mathcal{Q}}}$ as follows. By Lemma 6.17, there is an $\epsilon$ such that $0<\epsilon<r$ and $Q \subseteq \bigcup_{i=1}^{n} B_{x_{i},<r-\epsilon}^{d}$. Hence, for every $y \in Q$, there is an index $i$ such that $d\left(x_{i}, y\right)<r-\epsilon$, so $d\left(Q_{0}, y\right)<r-\epsilon$. Taking suprema over $y \in Q, d_{\mathcal{Q}}\left(Q_{0}, Q\right) \leq r-\epsilon<r$.

For every $a>0, d_{\mathcal{Q}}^{a}\left(Q_{0}, Q\right)=\min \left(a, d_{\mathcal{Q}}\left(Q, Q^{\prime}\right)\right)$ by Remark 6.4 , whence $B_{Q_{0},<r}^{d_{\mathcal{Q}}^{a}}=B_{Q_{0},<r}^{d_{\mathcal{Q}}}$ if $r \leq a$.

Theorem 6.19 (Algebraicity of Smyth hyperspaces). Let $X, d$ be an algebraic complete quasi-metric space, with a strong basis $\mathcal{B}$.

The space $\mathcal{Q} X, d_{\mathcal{Q}}$ is algebraic complete.

Every non-empty compact saturated set of the form $\uparrow\left\{x_{1}, \cdots, x_{n}\right\}$, where every $x_{i}$ is a center point, is a center point of $\mathcal{Q} X, d_{\mathcal{Q}}$, and those sets form a strong basis, even when each $x_{i}$ is taken from $\mathcal{B}$. The $d_{\mathcal{Q}}$-Scott topology coincides with the upper Vietoris topology.

Proof. We first recall that every algebraic complete quasi-metric space is continuous complete, and that every continuous quasi-metric space is sober. Then $\mathcal{Q}, d_{\mathcal{Q}}$ is complete by Proposition 6.9 , and $\uparrow\left\{x_{1}, \cdots, x_{n}\right\}$ (with $n \geq 1$ ) is a center point as soon as every $x_{i}$ is a center point, by Lemma 6.14 . We let $\mathcal{B}_{0}$ be the set of all center points of the form $\uparrow\left\{x_{1}, \cdots, x_{n}\right\}$ where $n \geq 1$ and each $x_{i}$ is in $\mathcal{B}$, and $\tau$ be the upper Vietoris topology on $\mathcal{Q} X$. The conclusion will follow from Lemma 5.20. Let us verify its assumptions.

(i) Let $Q_{0} \stackrel{\text { def }}{=} \uparrow\left\{x_{1}, \cdots, x_{n}\right\}$ where $x_{1}, \ldots, x_{n}$ are in $\mathcal{B}$ and $n \geq 1$. By Lemma $6.18, B_{Q_{0},<r}^{d_{\mathcal{Q}}}$ is equal to the upper Vietoris open set $\square\left(\bigcup_{i=1}^{n} B_{x_{i},<r}^{d}\right)$, for every $r>0$.

(ii) Let $\epsilon>0, Q \in \mathcal{Q} X$, and $\mathcal{U}$ be an upper Vietoris open neighborhood of $Q$. By definition of the latter topology, and since $\square U \cap \square V=\square(U \cap V)$ for all open subsets $U$ and $V$ of $X$, there is an open subset of $Q$ such that $Q \in \square U \subseteq \mathcal{U}$. We apply Lemma 6.16 in order to obtain finitely many points $z_{1}, \ldots, z_{p}$ in $\mathcal{B}$ and radii $t_{1}, \ldots, t_{p}>0$ such that $Q \subseteq \bigcup_{k=1}^{p} B_{z_{k},<t_{k}}^{d} \subseteq U$. By Lemma 6.17, there is a number $r$ such that $0<r \leq t_{1}, \cdots, t_{p}$ and such that $Q \subseteq \bigcup_{k=1}^{p} B_{z_{k}, t_{k}-r}^{d}$; we may also require that $r \leq \epsilon$. We apply Lemma 6.16 once again in order to obtain finitely many points $x_{1}, \ldots, x_{n}$ in $\mathcal{B}$ and radii $0<r_{1}, \ldots, r_{n}<r$ such that $Q \subseteq \bigcup_{j=1}^{n} B_{x_{j},<r_{j}}^{d} \subseteq \bigcup_{k=1}^{p} B_{z_{k}, t_{k}-r}^{d}$. Since $r_{j}<r$ for every $j, Q$ is included in $\bigcup_{j=1}^{n} B_{x_{j},<r}^{d}$. We verify that the latter is included in $U$. For every $z \in \bigcup_{j=1}^{n} B_{x_{j},<r}^{d}$, we have $d\left(x_{j}, z\right)<r$ for some $j, 1 \leq j \leq n$, and $x_{j}$ is in $\bigcup_{k=1}^{p} B_{z_{k},<t_{k}-r}^{d}$, so $d\left(z_{k}, x_{j}\right)<t_{k}-r$ for some $k$, 
$1 \leq k \leq p$. Therefore $d\left(z_{k}, z\right)<t_{k}-r+r=t_{k}$, showing that $z$ is in $B_{z_{k},<t_{k}}^{d}$, hence in $U$. This finishes to show that $\bigcup_{j=1}^{n} B_{x_{j},<r}^{d}$ is included in $U$, hence that $\square\left(\bigcup_{j=1}^{n} B_{x_{j},<r}^{d}\right)$ is included in $\square U$.

In summary, $Q \in \square\left(\bigcup_{j=1}^{n} B_{x_{j},<r}^{d}\right) \subseteq \square U \subseteq \mathcal{U}$. By Lemma 6.18, $\square\left(\bigcup_{j=1}^{n} B_{x_{j},<r}^{d}\right)$ is the open ball $B_{Q_{0},<r}^{d_{\mathcal{Q}}}$, and this finishes to establish (ii).

(iii) The specialization preordering of the upper Vietoris topology is reverse inclusion. Although this is well-known, here is a quick proof. If $Q \supseteq Q^{\prime}$, then certainly $Q \in \square U$ implies $Q^{\prime} \in \square U$, for every open set $U$. Conversely, $Q \in \square U$ implies $Q^{\prime} \in \square U$, for every open set $U$, then $Q^{\prime}$ is included in every open neighborhood of $Q$, hence in their intersection, which happens to be $Q$, since $Q$ is saturated. Now the specialization preordering of the $d_{\mathcal{Q}}$ Scott topology, $\leq^{d_{\mathcal{Q}}}$, is also reverse inclusion by Lemma 6.6.

Remark 6.20. The same result holds for $d_{\mathcal{Q}}^{a}$, for every $a \in \mathbb{R}_{+}$, $a>0$ : when $X, d$ is standard algebraic, $\mathcal{Q} X, d_{\mathcal{Q}}^{a}$ is algebraic complete, with the same strong basis. The proof is mostly the same. We invoke Remark 6.10 instead of Proposition 6.9 in order to obtain completeness, then Remark 6.15 instead of Lemma 6.14 for center points, and finally Remark 6.7 instead of by Lemma 6.6 concerning the specialization preordering. The rare differences are that, in (i), $B_{Q_{0},<r}^{d_{Q}^{a}}$ is only equal to $\square\left(\bigcup_{i=1}^{n} B_{x_{i},<r}^{d}\right)$ when $r<a$; otherwise it is the whole of $\mathcal{Q} X$. As a consequence, in proving (ii), we need to make sure that $r$ is also smaller than or equal to a, not just to $\epsilon, t_{1}, \ldots, t_{p}$.

\subsection{Continuity}

We proceed exactly as in Section 5.5 for the Hoare hyperspaces.

We start with an easy fact.

Fact 6.21. For every topological space $Y$, for every subset $A$ of $Y$, for every monotonic map $h: Y \rightarrow \mathbb{R} \cup\{-\infty,+\infty\}, \inf _{y \in A} h(y)=\inf _{y \in \uparrow A} h(y)$.

Writing $f[Q]$ for the image of $Q$ by $f$, we have the following.

Lemma 6.22. Let $X, d$ and $Y, \partial$ be two continuous complete quasi-metric spaces, and $f: X, d \mapsto Y, \partial$ be a 1-Lipschitz continuous map. The map $\mathcal{Q} f: \mathcal{Q} X, d_{\mathcal{Q}} \rightarrow \mathcal{Q} Y, d_{\mathcal{Q}}$ defined by $\mathcal{Q} f(Q) \stackrel{\text { def }}{=} \uparrow f[Q]$ is 1-Lipschitz continuous. Moreover, $F_{\mathcal{Q} f(Q)}=\mathbf{P} f\left(F_{Q}\right)$ for every $Q \in \mathcal{Q} X$.

Similarly with $d_{\mathcal{Q}}^{a}$ instead of $d_{\mathcal{Q}}$. 
Proof. We first check that $F_{\mathcal{Q} f(Q)}=\mathbf{P} f\left(F_{Q}\right)$. For every $h \in \mathcal{L} X, F_{\mathcal{Q} f(Q)}(h)=$ $\inf _{y \in \uparrow f[Q]} h(y)=\inf _{y \in f[Q]} h(y)$ by Fact 6.21. That is equal to $\inf _{x \in X} h(f(x))$. We also have $\mathbf{P} f\left(F_{Q}\right)(h)=F_{Q}(h \circ f)=\inf _{x \in X} h(f(x))$. Therefore $F_{\mathcal{Q} f(Q)}=$ $\mathbf{P} f\left(F_{Q}\right)$.

By Lemma 4.21, $\mathbf{P} f$ is 1-Lipschitz, so $\mathbf{B}^{1}(\mathbf{P} f)$ is monotonic. Also, $\mathbf{P} f$ maps normalized discrete superlinear previsions to normalized discrete superlinear previsions. By Proposition $6.9, \mathcal{Q} X, d_{\mathcal{Q}}$ is complete, hence through the isometry of Proposition 5.8, the corresponding spaces of normalized discrete superlinear previsions are complete as well. Moreover, directed suprema of formal balls are computed as naive suprema. By Lemma $4.22, \mathbf{B}^{1}(\mathbf{P} f)$ preserves naive suprema, hence all directed suprema. It must therefore be Scott-continuous. Hence $\mathcal{Q} f$ is 1 -Lipschitz continuous.

In the case of $d_{\mathcal{Q}}^{a}$, the argument is the same, except that we use Remark 6.10 instead of Proposition 6.9.

Let $X, d$ be a continuous complete quasi-metric space. There is an algebraic complete quasi-metric space $Y, \partial$ and two 1-Lipschitz continuous maps $r: Y, \partial \rightarrow X, d$ and $s: X, d \rightarrow Y, \partial$ such that $r \circ s=\mathrm{id}_{X}$.

By Lemma 6.22, $\mathcal{Q} r$ and $\mathcal{Q} s$ are also 1-Lipschitz continuous, and clearly $\mathcal{Q} r \circ \mathcal{Q} s=\operatorname{id}_{\mathcal{Q} X}$, so $\mathcal{Q} X, d_{\mathcal{Q}}$ is a 1-Lipschitz continuous retract of $\mathcal{Q} Y, \partial_{\mathcal{Q}}$. (Similarly with $d_{\mathcal{Q}}^{a}$ and $\partial_{\mathcal{Q}}^{a}$.) Theorem 6.19 states that $\mathcal{Q} Y, \partial_{\mathcal{Q}}$ (resp., $\partial_{\mathcal{Q}}^{a}$, using Remark 6.20 instead) is algebraic complete, whence the following.

Theorem 6.23 (Continuity for the Smyth hyperspace). Let $X, d$ be a continuous complete quasi-metric space. The quasi-metric spaces $\mathcal{Q} X, d_{\mathcal{Q}}$ and $\mathcal{Q} X, d_{\mathcal{Q}}^{a}\left(a \in \mathbb{R}_{+}, a>0\right)$ are continuous complete.

Together with Lemma 6.22, and Theorem 6.19 for the algebraic case, we obtain the following.

Corollary 6.24. $\mathcal{Q}, d_{\mathcal{Q}}$ defines an endofunctor on the category of continuous complete quasi-metric spaces and 1-Lipschitz continuous map. Similarly with $d_{\mathcal{Q}}^{a}$ instead of $d_{\mathcal{Q}}(a>0)$, or with algebraic instead of continuous.

6.6. The upper Vietoris topology

Lemma 6.25. Let $X, d$ be a standard quasi-metric space that is sober in its $d$-Scott topology, and $a, a^{\prime}>0$ with $a \leq a^{\prime}$. We have the following inclusion of topologies:

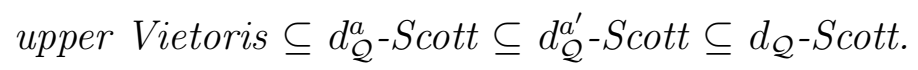


Proof. Considering Lemma 3.6, this is a consequence of Proposition 5.27. Note that the latter applies because directed suprema of formal balls are indeed naive suprema, due to Proposition 6.9.

Theorem 6.26 ( $d_{\mathcal{Q}}$ quasi-metrizes the upper Vietoris topology). Let $X, d$ be a continuous complete quasi-metric space. The $d_{\mathcal{Q}}$-Scott topology, the $d_{\mathcal{Q}}^{a}$-Scott topology, for every $a \in \mathbb{R}_{+}, a>0$, and the upper Vietoris topology all coincide on $\mathcal{Q} X$.

Proof. The proof is as for Theorem 5.30. $X, d$ is the 1-Lipschitz continuous retract of an algebraic complete quasi-metric space $Y, \partial$. Call $s: X \rightarrow Y$ the section and $r: Y \rightarrow X$ the retraction. We confuse $\mathcal{Q} X$ with the corresponding space of discrete sublinear previsions. Then $\mathbf{P} s$ and $\mathbf{P} r$ form a 1-Lipschitz continuous section-retraction pair by Lemma 4.21, and in particular $\mathbf{P} s$ is an embedding of $\mathcal{Q} X$ into $\mathcal{Q} Y$ with their $d_{\mathcal{Q}}$-Scott topologies (similarly with $d_{\mathcal{Q}}^{a}$ in place of $d_{\mathcal{Q}}$ ). However, $s$ and $r$ are also just continuous, since 1-Lipschitz continuous maps between standard quasi-metric spaces are continuous. Therefore $\mathbf{P} s$ and $\mathbf{P} r$ also form a section-retraction pair between the same spaces, this time with their weak topologies (as spaces of previsions), by Fact 5.28, that is, with their upper Vietoris topologies, by Lemma 3.6. (Recall that $X$ and $Y$ are sober since continuous complete.) The final claim of Theorem 6.19 is that the two topologies on $\mathcal{Q} Y$ are the same, since $Y, \partial$ is algebraic complete. Fact 5.29 then implies that the two topologies on $\mathcal{Q} X$ are the same as well.

\section{Open Problems}

1. We have obtained that $\mathcal{H}_{0}, \mathcal{H}$ all map continuous complete spaces to continuous complete spaces, and algebraic complete spaces to algebraic complete spaces. Is the Hoare hyperspace of a (merely) complete quasimetric space complete again? Proposition 5.12 does not answer the question. Note that a similar question for Smyth hyperspaces has a full answer, see Proposition 6.9 and Remark 6.11.

2. Is Smyth-completeness preserved by $\mathcal{H}_{0}, \mathcal{H}, \mathcal{Q}$ ?

3. For general spaces of previsions, it is frustrating that Proposition 4.25 is only a conditional completeness theorem. Is there a general argument to the effect that its condition on supports is always true, namely that 
every element of $Z$ supported on $V_{1 / 2^{n}}$ for every $n \in \mathbb{N}$ is supported on $X ?$

\section{References}

[1] Bonsangue, M. M., van Breugel, F., Rutten, J. J. M. M., 1998. Generalized metric spaces: Completion, topology, and powerdomains via the Yoneda embedding. Theoretical Computer Science 193, 1-51.

[2] de Brecht, M., Goubault-Larrecq, J., Jia, X., Lyu, Z., 2019. Domaincomplete and LCS-complete spaces. Electronic Notes in Theoretical Computer Science 345, 3-35, proc. 8th International Symposium on Domain Theory (ISDT'19), also available as arXiv report 1902.11142 [math.GN].

[3] Edalat, A., Heckmann, R., 1998. A computational model for metric spaces. Theoretical Computer Science 193, 53-73.

[4] Escardó, M. H., 1998. Properly injective spaces and function spaces. Topology and Its Applications 89 (1-2).

[5] Gierz, G., Hofmann, K. H., Keimel, K., Lawson, J. D., Mislove, M., Scott, D. S., 2003. Continuous Lattices and Domains. Vol. 93 of Encyclopedia of Mathematics and its Applications. Cambridge University Press.

[6] Goubault-Larrecq, J., Sep. 2007. Continuous previsions. In: Duparc, J., Henzinger, T. A. (Eds.), Proceedings of the 16th Annual EACSL Conference on Computer Science Logic (CSL'07). Springer-Verlag LNCS 4646, Lausanne, Switzerland, pp. 542-557.

[7] Goubault-Larrecq, J., 2013. Non-Hausdorff Topology and Domain Theory-Selected Topics in Point-Set Topology. Vol. 22 of New Mathematical Monographs. Cambridge University Press.

[8] Goubault-Larrecq, J., Jan. 2015. Full abstraction for non-deterministic and probabilistic extensions of PCF I: the angelic cases. Journal of Logic and Algebraic Methods in Programming 84 (1), 155-184. URL http://www.lsv.ens-cachan.fr/Publis/PAPERS/PDF/ jgl-jlap14.pdf 
[9] Goubault-Larrecq, J., 2017. Complete quasi-metrics for hyperspaces, continuous valuations, and previsions, arXiv 1707.03784, v2, https: //arxiv.org/abs/1707.03784; v6, July 2020.

[10] Goubault-Larrecq, J., 2019. Formal ball monads. Topology and its Applications 263, 372-391.

[11] Goubault-Larrecq, J., 2020. Some topological properties of spaces of Lipschitz continuous maps on quasi-metric spaces. Topology and its Applications 282, 107281.

URL https://www.sciencedirect.com/science/article/pii/ S0166864120302248

[12] Goubault-Larrecq, J., 2021. Kantorovich-Rubinshtein quasi-metrics I: Spaces of measures and of continuous valuations. Topology and its Applications 295, 107673.

URL https://www.sciencedirect.com/science/article/pii/ S0166864121000870

[13] Goubault-Larrecq, J., Ng, K. M., Oct. 2017. A few notes on formal balls. Logical Methods in Computer Science 13 (4:18), 1-34, special issue of Domain Theory and Applications (the Domains XII Workshop), Cork, Ireland, August 2015.

[14] Johnstone, P. T., 1981. Scott is not always sober. In: Continuous Lattices. Vol. 871 of Lecture Notes in Mathematics. Springer Verlag, Berlin and New York, pp. 282-283.

[15] Kostanek, M., Waszkiewicz, P., 2010. The formal ball model for $\mathcal{Q}$ categories. Mathematical Structures in Computer Science 21 (1), 1-24.

[16] Scott, D. S., 1972. Continuous lattices. In: Lawvere, F. W. (Ed.), Toposes, Algebraic Geometry and Logic. Vol. 274 of Lecture Notes in Mathematics. Springer, Berlin, Heidelberg, pp. 97-236.

[17] Weihrauch, K., Schreiber, U., 1981. Embedding metric spaces into cpo's. Theoretical Computer Science 16 (1), 5-24. 